\title{
ARES I Aerodynamic Testing at the NASA Langley Unitary Plan Wind Tunnel
}

\author{
Gary E. Erickson ${ }^{1}$ and Floyd J. Wilcox ${ }^{2}$ \\ NASA Langley Research Center, Hampton, VA, 23681
}

\begin{abstract}
Small-scale force and moment and pressure models based on the outer mold lines of the Ares I design analysis cycle crew launch vehicle were tested in the NASA Langley Research Center Unitary Plan Wind Tunnel from May 2006 to September 2009. The test objectives were to establish supersonic ascent aerodynamic databases and to obtain force and moment, surface pressure, and longitudinal line-load distributions for comparison to computational predictions. Test data were obtained at low through high supersonic Mach numbers for ranges of the Reynolds number, angle of attack, and roll angle. This paper focuses on (1) the sensitivity of the supersonic aerodynamic characteristics to selected protuberances, outer mold line changes, and wind tunnel boundary layer transition techniques, (2) comparisons of experimental data to computational predictions, and (3) data reproducibility. The experimental data obtained in the Unitary Plan Wind Tunnel captured the effects of evolutionary changes to the Ares I crew launch vehicle, exhibited good agreement with predictions, and displayed satisfactory within-test and tunnel-to-tunnel data reproducibility.
\end{abstract}

\section{Nomenclature}

$\begin{array}{ll}\text { ADAC } & =\text { Ares design analysis cycle } \\ A L A S & =\text { Alternate launch abort system } \\ B D M & =\text { Booster deceleration motors } \\ B M C & =\text { Balance moment center } \\ B P C & =\text { Boost protective cover } \\ C_{A} & =\text { Axial force coefficient } \\ C l & =\text { Rolling moment coefficient } \\ C_{m} & =\text { Pitching moment coefficient } \\ C_{n} & =\text { Yawing moment coefficient } \\ C_{N} & =\text { Normal force coefficient } \\ C_{p} & =\text { Pressure coefficient } \\ C_{Y} & =\text { Side force coefficient } \\ C E V & =\text { Crew exploration vehicle } \\ C F D & =\text { Computational fluid dynamics } \\ C L V & =\text { Crew launch vehicle } \\ C M & =\text { Crew module } \\ d & =\text { Model reference diameter } \\ D A C & =\text { Design analysis cycle } \\ E S M D & =\text { Exploration Systems Mission Directorate } \\ E S P & =\text { Electronically-scanned pressure } \\ F S & =\text { First stage } \\ F . S . & =\text { Full scale } \\ I L V & =\text { Integrated launch vehicle } \\ I S & =\text { Interstage } \\ I T A R & =\text { International Traffic in Arms Regulations } \\ L & =\text { Model length } \\ L a R C & =\text { Langley Research Center } \\ L A S & =\text { Launch abort system } \\ & \end{array}$

${ }_{2}^{1}$ Aerospace Engineer, Configuration Aerodynamics Branch, Mail Stop 499.

${ }^{2}$ Aerospace Engineer, Configuration Aerodynamics Branch, Mail Stop 499. 


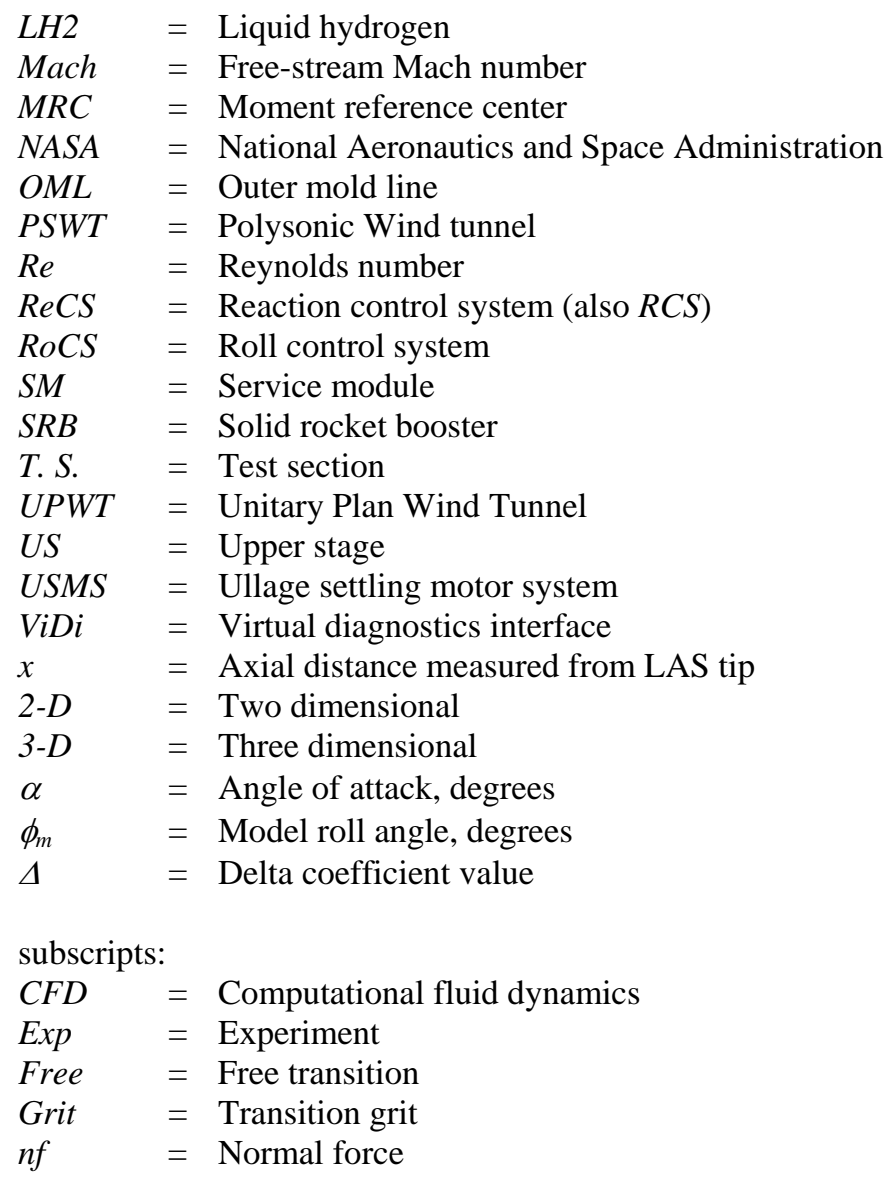

\section{Introduction}

Following the completion of the National Aeronautics and Space Administration's (NASA) Exploration Systems F Architecture Study ${ }^{1}$ in August 2004 for the NASA Exploration Systems Mission Directorate (ESMD), design and development work was initiated on the Ares I crew launch vehicle (CLV) as part of the NASA Constellation program $^{2}$. The Ares I CLV is intended to launch astronauts via the Orion crew exploration vehicle (CEV) to low earth orbit and rendezvous with either the International Space Station or the ESMD's earth departure stage for lunar or other future missions beyond low Earth orbit ${ }^{2}$. A sketch of the Ares I integrated launch vehicle (ILV) is shown in fig. 1. Wind tunnel testing is the primary source for databases in the Ares I design analysis cycle (ADAC) process ${ }^{3}$, and experiments serve a significant role in the validation of the predictions from computational fluid dynamics (CFD) methods ${ }^{4}$. At the outset, CFD was intended to be the primary means of calculating vehicle loads. A NASAwide Ares I aerodynamics team conducted a series of wind tunnel tests of small-scale models to establish the requisite experimental databases at subsonic through supersonic speeds ${ }^{4}$. This paper provides highlights of supersonic wind tunnel testing in the NASA Langley Research Center (NASA LaRC) Unitary Plan Wind Tunnel (UPWT) of 0.01-scale force and moment models based on the evolutionary changes made to the Ares I outer mold lines (OML) designated ADAC-1, ADAC-2A, ADAC-2B, and ADAC-3 and a 0.01-scale dedicated pressure model based on the Ares I ADAC-1 OML. The primary objectives of these tests were supersonic ascent aerodynamic database development and CFD method validation. The technical discussion will center on several of the significant aerodynamic effects caused by major protuberances and OML changes on the Ares I vehicle, sensitivity to wind tunnel testing techniques to promote boundary layer transition, comparisons of the experimental data to the predicted effects using the CFD code USM3D ${ }^{4}$, and within-test and tunnel-to-tunnel data reproducibility. 


\section{Objectives}

The objectives of this report include a summary of the incremental effects on the forces and moments of (1) a launch abort system (LAS) flare, (2) modifications to the boost protective cover (BPC) OML over the CEV, (3) a liquid hydrogen (LH2) feedline fairing, (4) changes to the size, number and location of booster deceleration motors (BDM), and (5) an interstage strake. The detailed surface static pressure distributions and longitudinal line-load distributions obtained on the dedicated axisymmetric pressure model are also presented. Representative results from testing the pressure model with a LAS flare and with a bolt-on solid plume to simulate the solid rocket booster (SRB) exhaust plume at a high supersonic Mach number are shown. The sensitivity of the force and moment and surface pressure data to the application of artificial surface roughness to promote boundary layer transition and the use of a sublimating chemical technique ${ }^{5}$ to visualize the surface-flow behavior are discussed. The experimental forces and moments and surface static pressure and line-load distributions are compared to CFD predictions using the code USM3D ${ }^{4}$. Examples of using a real-time three-dimensional (3-D) virtual diagnostics interface (ViDi) application $^{6}$ to compare experimental schlieren flow visualization ${ }^{6}$ and surface pressure distributions to CFD predictions of the density contours and surface pressures are shown. Within-test data repeatability is discussed, and tunnel-to-tunnel data reproducibility is assessed by comparing selected configurations via force and moment and pressure data obtained in the NASA LaRC UPWT and the Boeing Polysonic Wind Tunnel (PSWT).

\section{Test Information}

\section{A. Models and Instrumentation}

The Ares I OMLs that were successively tested in the NASA LaRC UPWT from 2006 to 2009 are shown in the wind tunnel model installation photographs in fig. 2 through fig. 6. Internal six-component strain-gage balances were used to measure the forces and moments on the Ares I ADAC-1 through ADAC-3 models. The ADAC-1, ADAC-2A, and ADAC-2B models used existing balances in the NASA LaRC inventory that were appropriate for testing of slender configurations at supersonic speeds ${ }^{7}$. The ADAC-3 model used a new balance that was designed and fabricated specifically for Ares I testing based on experience gained from the previous test programs ${ }^{8}$. The model pitch attitude was determined using an accelerometer mounted in the main support system pitch mechanism with corrections applied to account for tunnel flow angularity and aeroelastic deflections of the model/balance/sting assembly. Model roll angle was measured using an automated roll coupling. The ADAC-1 dedicated pressure model was primarily tested in the clean configuration (all protuberances off) and incorporated a total of 146 surface static pressure orifices, with the majority of pressures located along the 0 -degree azimuth or top centerline. The pressure orifices were connected to four external electronically-scanned pressure (ESP) modules. Pitch and roll attitude for the dedicated pressure model was determined using onboard accelerometers with corrections applied to account for tunnel flow angularity. The aerodynamic force and moment coefficients were computed in body, stability, missile, and vertical plane axis systems ${ }^{7,8}$. The moment coefficients were computed using a moment reference center (MRC) at the gimble point of the SRB nozzle.

\section{B. Wind Tunnel Facility}

The wind tunnel testing was conducted from low to high supersonic Mach numbers in the NASA LaRC UPWT, which is a continuous-flow, variable-pressure supersonic wind tunnel. The tunnel contains two test sections which are approximately 4 feet square and 7 feet long. Each test section encompasses only part of the Mach number range of the tunnel. Consequently, testing of all Ares I wind tunnel models required a dedicated installation in each test section in order to cover the desired range of Mach number. The nozzle ahead of each test section consists of an asymmetric sliding block which allows continuous Mach number variation during tunnel operations in the "low" Mach number test section 1 (T. S. 1) and in the "high" Mach number test section 2 (T. S. 2). A complete description of the facility is provided in ref. 9. This report compares selected results obtained in the UPWT to data from the Boeing PSWT, which is a blow-down facility with nominal 4-foot by 4-foot test section. A detailed description of the Boeing facility is included in ref. 3.

\section{Discussion of Results}

\section{A. Ares I ADAC-1 Force and Moment Data}

This section will focus on the aerodynamic effects associated with a LAS flare, which was a proposed covering for the LAS motor nozzles on the ADAC-1. Figure 7 presents a sketch of the Ares I ADAC- 1 configuration with several key protuberances identified. The transition grit strategies that were used in UPWT T. S. 1 are illustrated in 
fig. 8. Initial testing was performed with a single band of randomly applied \#45 grit on the LAS tower, and a second band of \#50 grit was added based on the early test results. The LAS flare was initially tested in a fixed baseline location as illustrated in fig. 9. However, a second LAS tower was fabricated with a moveable flare to assess an aerodynamic anomaly that was observed during the initial wind-on runs. Two alternate LAS skirt locations aft of the baseline position are shown in fig. 9. Figure 10 shows close-up photographs of the 0.01-scale Ares I ADAC-1 force and moment model with the LAS skirt in baseline and aft positions in UPWT T. S. 1. Figure 11 presents two schlieren flow visualization video frames acquired with the baseline LAS flare position that illustrate the aerodynamic anomaly that was observed at the lower supersonic Mach numbers in UPWT T. S. 1 . The video frames were obtained at the same angle of attack but depict a bimodal flow condition arising from unsteady shock-induced flow separation at the flare. The two conditions are referred to as "low drag" and "high drag" since the size of the wake shed from the flare had a significant effect on the balance axial force measurements. It is speculated that the low-drag condition is the result of the flow separating from the LAS flare and enveloping the CEV, or capsule, in a wake. The high-drag condition is associated with the flow expanding around the LAS flare and reattaching on the LAS cylinder so that the capsule is not in a wake. Insufficient data were acquired, however, to definitively state that this effect was solely caused by the Reynolds number. In fact, the flow "popping” between apparent re-attached and separated flow states downstream of the flare occurred at certain combinations of the angle of attack, Mach number, and Reynolds number. This phenomenon was also sensitive to the direction of movement of the model support system (hysteresis), the transition grit application, and the location of the LAS flare. In general, the "high-drag" flow state could be achieved by increasing the angle of attack beyond a certain critical value, increasing the Mach number at a given Reynolds number, increasing the Reynolds number at a given Mach number, or adding a second band of transition grit on the LAS tower. Displacing the flare aft of the baseline position also caused a significant effect on the overall axial force. The unsteady aerodynamics caused by the LAS flare in the baseline position was also observed in supersonic testing of the ADAC-1 model in the Boeing PSWT and is described in ref. 10. Figures 12 through 16 provide representative results illustrating these respective trends. For example, fig. 12 shows the effect of increasing the Reynolds number on the axial force coefficient variation with the angle of attack at a lower supersonic Mach number in UPWT T. S. 1. A sufficient increase in the Reynolds number eliminates the bimodal state that promotes an abrupt increase in the axial force coefficient at the lower Reynolds numbers. A similar effect on the axial force coefficient is observed by increasing the Mach number at a selected lower Reynolds number condition as shown in fig. 13. Figure 14 indicates that the addition of a single band of \#50 grit ahead of the original single band of \#45 grit on the LAS tower dramatically affects the axial force characteristics at a lower Reynolds number and selected Mach number condition and also effectively eliminates the bimodal flow state. Figure 15 shows a significant reduction in the effect of a second band of transition at a higher Reynolds number condition. Finally, fig. 16. shows an aft displacement of the LAS flare promotes a large decrease in the axial force coefficient through the range of angle of attack tested in UPWT T. S. 1 at a selected lower Reynolds number and Mach number. The results in fig. 16 were obtained with the additional grit band on the LAS. Consequently, the mid and aft positions may cause a single-mode separated flow state downstream of the flare that persists throughout the range of angle of attack.

\section{B. Ares I ADAC-1 Pressure Data}

Highlights from the testing of the 0.01-scale Ares I ADAC-1 pressure model with all protuberances removed (axisymmetric clean configuration) and with a movable LAS flare and a simulated SRB exhaust plume are presented in this section. Figure 17 shows the pressure tap layout for the dedicated pressure model, which was fabricated for testing in both the NASA LaRC and the Boeing PSWT facilities. Note that the clean configuration did not include the LAS flare that is shown in fig. 17. The distribution of the discrete orifices on the wind tunnel model was based on pre-test CFD predictions and was intended to capture the key features of the surface pressure field at the subsonic through supersonic test conditions. The symmetry of the clean configuration motivated a single row of high-density pressure taps along the top centerline. The distribution of the circumferential surface static pressures at any of the model axial locations corresponding to a pressure tap in this row could be estimated by acquiring data in a roll sweep from 0 degrees to 180 degrees at a selected (fixed) angle of attack. In addition, the longitudinal load distribution could be estimated from the roll sweep data by integrating these pressures at each of the 105 axial locations on the model and by assuming symmetry of the left and right halves of the model. This experimental approach was possible, since the NASA LaRC and Boeing PSWT facilities were capable of remotely controlling the roll angle during the tunnel operation. It was not within the scope of the present experiment to obtain line-load information on the baseline ADAC-1 with all protuberances, since this asymmetric configuration would have required either prohibitively dense distributions of pressure taps on the model to estimate the line load distributions or the use of a global pressure-sensitive paint technique ${ }^{5}$, which was not considered sufficiently mature at UPWT for

4

American Institute of Aeronautics and Astronautics 
this application. The priority test configuration corresponded to the clean configuration. Small subsets of pressure data were also acquired with a flare that was tested in three positions on the LAS tower and with a bolt-on solid plume that was tested at a high supersonic Mach number.

The initial phase of testing was dedicated to a transition grit study to determine the sensitivity of the pressure distributions to different grit strategies. A chemical sublimation technique ${ }^{5}$ was used in an attempt to visualize regions of boundary layer transition on the model. Figure 18 shows the different transition grit applications that were investigated during this initial testing in UPWT T. S. 1. The model was also tested without grit, which corresponded to the "free-transition" configuration. The pattern featuring the two bands of \#180 grit was tested for comparison to results obtained in the Boeing PSWT using the same transition grit pattern. Figure 19 presents selected sublimation patterns that were recorded at specific time intervals using a digital camera located outside the test section and positioned near a selected window in the test section side wall. The sublimation results did not identify a grit pattern that was clearly effective in promoting boundary layer transition. Sublimation occurred slowly along the aft section of the LAS tower and the forward section of the CEV, which suggested a region of separated or re-circulating flow. The latter phenomenon was confirmed in limited flow visualization runs using a colored alcohol technique ${ }^{11}$ to visualize the local surface flow behavior. Bottles of colored alcohol were located outside the test section and were connected to selected pressure lines attached to orifices on the 0-degree azimuth on the LAS tower and CEV. Observation of the alcohol that was entrained into the low pressure region along the LAS tower and CEV showed locally separated and reversed flow in the region where the rate of sublimation was low. The schlieren photographs obtained at different Mach numbers in UPWT T. S. 1 in fig. 20 reveal a region of apparent shockinduced flow separation at a lower supersonic Mach number. This phenomenon is not as obvious at a higher supersonic Mach number, however. The pressure distribution and delta coefficient comparisons in fig. 21 are sensitive to the transition grit arrangement. However, the chemical sublimation flow visualization did not provide conclusive evidence that any of the distributions were associated with a turbulent boundary layer. The default grit pattern that was used for the remainder of the testing featured the two transition bands on the LAS tower consisting of randomly-applied \#50 and \#45 grit as shown in the sketch in fig. 18 and in the model installation photograph in fig. 22. This is the same grit strategy that was illustrated in fig. 8 in section IV-A for the ADAC- 1 force and moment model testing and was effective in mitigating an unsteady shock-induced flow separation phenomenon when the LAS flare was installed.

Figure 23 shows a comparison of the experimental schlieren flow visualization patterns from UPWT T. S. 1 and CFD-predicted density contours. A virtual rendering of the model using the ViDi software application ${ }^{6}$ is overlayed onto the actual model image. The salient features of the off-surface flow consisting of shock waves emanating from several locations on the vehicle are similar and effectively captured in both the experiment and CFD. Figure 24 is a snapshot of a real-time display of the two-dimensional (2-D) experimental and CFD pressure distributions superimposed onto 3-D renderings of specific sections of the model with color-coded CFD surface static pressure maps. The experimental data and CFD predictions are displayed in black and red, respectively, and differences are shown in yellow. The ViDi output was a primary real-time data display that provided point-by-point feedback on the quality of the experimental pressure measurements. The experimental line-load distributions are compared to the CFD predictions in fig. 25. Figure 26 and fig. 27 present tunnel-to-tunnel comparisons of the pressure distributions and line-load distributions, respectively, obtained on the same model in the NASA LaRC UPWT T. S. 1 and in the Boeing PSWT. In general, the UPWT-to-CFD and tunnel-to-tunnel comparisons reveal good agreement. The largest differences that were observed typically occurred along the CEV and the first stage aft skirt (see fig. 7). However, the surface pressure comparisons do not reveal any significant sources of data variability between the two tunnels. There is no current metric available to quantify acceptable levels of agreement between experiment and CFD or tunnel-to-tunnel. In addition, confirmation of the boundary layer state on the wind tunnel models remained elusive, and a departure from the fully-turbulent boundary layer assumed in the CFD computations is a possible contributor to differences between experiment and CFD in the surface pressure and line-load distributions.

The pressure model was tested with a conical LAS flare at the three positions previously shown in fig. 9 and fig. 10 corresponding to the 0.01-scale Ares I ADAC-1 force and moment model in Section IV-A. Test results were obtained in roll angle sweeps at selected angles of attack. Representative results corresponding to the baseline flare position and with the flare positioned farther aft on the LAS tower are presented in fig. 28 and fig. 29, respectively. Results obtained with the model upright and at a selected roll angle are shown in both figures, and the flare-off case is shown for reference in all plots. The LAS flare has the largest effect on the downstream local surface pressures 
along the LAS and CEV, and this effect increases as the flare is positioned farther aft on the LAS. The measured pressure distributions for the baseline flare position indicate that the sign of the pressure coefficient increments (flare on minus flare off) along the LAS and CEV at certain angles of attack is sensitive to the roll angle. This may be an underlying feature of the unsteady bimodal flow phenomenon that was observed on the ADAC- 1 force and moment model in section IV-A. The sign change in the pressure differences due to roll angle was not observed with the flare in the mid or aft positions.

Figure 30 shows a photograph of the ADAC-1 pressure model with a bolt-on solid plume component installed in UPWT T. S. 2. The solid plume was intended to simulate the SRB exhaust plume and potential plume-induced flow separation effects during ascent at a high supersonic Mach number. A schlieren flow visualization image obtained with the solid plume installed is shown in fig. 31. The surface static pressure distributions presented in fig. 32 indicate the presence of the solid plume is manifested as a pressure rise along approximately the aft 10 percent of the body length.

\section{Ares I ADAC-2A Force and Moment Data}

The contributions of the BPC OML and the BDM covers to the supersonic aerodynamic characteristics of the Ares I ADAC-2A are highlighted in this section. A sketch of the Ares I ADAC-2A configuration with protuberances is presented in fig. 33. The LAS flare on the Ares I ADAC-1 was eliminated on the ADAC-2A, and the four abort motor nozzles were simulated on the LAS tower. OML changes to BPC over the CEV on the ADAC1 resulted in a so-called "party-hat" design on the ADAC-2A. The four BDM covers positioned on the interstage (IS) upstream of the frustum were also enlarged on the ADAC-2A. The removal of the LAS flare eliminated the aerodynamic anomaly observed on the ADAC- 1 that was characterized by the bimodal flow phenomenon described in section IV-A. The party-hat OML and the enlarged BDM covers were plausible sources of increased axial force encountered on the ADAC-2A. The abrupt surface angle changes in the party-hat geometry caused the development of multiple shock waves as shown in the schlieren flow visualization image in fig. 34. This complex shock system is consistent with higher axial force levels and is a serious CEV acoustic loads issue ${ }^{11}$. The flow separation from this asymmetric configuration with multiple protuberances in combination with the flexible balance and sting assembly yielded a dynamic model response at the higher angles of attack in UPWT T. S. 1. A modified ogive BPC (designated ALAS-11) was fabricated during the UPWT testing, and close-up photographs of the baseline and modified configurations installed in UPWT T. S. 1 are compared in fig. 35. The ALAS-11 design also featured a bulbous fairing in the vicinity of the LAS nozzles. The schlieren flow visualization results in fig. 36 reveal a mitigation of the multiple shock pattern observed on the party-hat design, and significant model dynamics were no longer observed. In addition, the OML modification significantly decreased the axial force at all supersonic Mach numbers tested in UPWT T. S. 1 and T. S. 2. A typical comparison of the longitudinal aerodynamic characteristics with the baseline and ALAS-11 BPC OMLs is shown in fig. 37.

Figure 38 shows two close-up views of the interstage of the ADAC-2A model and the four large, diagonallyopposed BDM covers. The BDM covers were situated upstream of the frustum, which marked the transition between the first and upper stages. The covers were positioned 90 degrees apart and rotated 45 degrees with respect to the vertical and horizontal axes of the model. Schlieren video recordings clearly showed an unsteady shockinduced flow separation downstream of the BDM covers that was similar to the LAS flare bimodal phenomenon cited in section IV-A on the ADAC-1 model. This phenomenon was eliminated when the BDM covers were removed from the model. The longitudinal aerodynamic coefficients presented in fig. 39 indicate that the BDMs represent a significant percentage of the total axial force increments caused by the vehicle protuberances.

A second, identical 0.01-scale model of the Ares I ADAC-2A was tested in the Boeing PSWT in parallel with the UPWT testing. Figure 40 presents a tunnel-to-tunnel comparison of the six-component aerodynamic force and moment coefficients obtained in roll sweeps on the baseline configuration at a selected angle of attack and supersonic Mach number. The aerodynamic coefficients presented in this plot were computed in the body-axis system $^{7,8}$. A roll sweep in the Boeing facility consisted of two separate runs in order to acquire data over the full range of roll angle. The data generally show favorable agreement between the tunnels for all six components. Similar comparisons were obtained on the clean configuration, which provided confidence that each facility yielded consistent estimates of the aerodynamic effects of the full set of protuberances and of selected protuberance subsets. 


\section{Ares I ADAC-2B Force and Moment Data}

This section will focus on the aerodynamic effects of OML and protuberance changes that were implemented on the Ares I ADAC-2B. The BPC OML changed from the party hat design on the ADAC-2A to an ogive shape on the ADAC-2B as presented in fig. 41. Computer-aided design (CAD) renderings and close-up photographs comparing the BPC OMLs on the Ares I ADAC-2A and ADAC-2B are shown in fig. 42. The four large BDM covers on the ADAC-2A were removed from the IS and replaced with eight smaller BDM covers positioned on the first stage aft skirt of the ADAC-2B as illustrated in fig. 41. CAD renderings and a wind tunnel installation photograph in fig. 43 show the changes to the BDM covers on the ADAC-2B. The favorable results obtained with the ALAS-11 and the undesirable effects that were attributed to the BDM covers on the ADAC-2A model discussed in section IV-C contributed to the design changes on the ADAC-2B. The BPC OML change and the relocation and reconfiguration of the BDM covers are primary contributors to the large decrease in the axial force coefficient at supersonic speeds shown in fig. 44.

Figure 45 shows the reshaping of the LH2 feedline fairing on the ADAC-2B compared to the ADAC-2A. The LH2 feedline fairing is located at a 90-degree azimuth on the upper stage and is a more prominent protuberance on the ADAC-2B. Figure 46 presents a close-up photograph of the fairing and surrounding protuberances on the ADAC-2B model installed in UPWT T. S. 1. Figure 47 compares the lateral-directional aerodynamic coefficients on the ADAC-2A and ADAC-2B obtained in roll angle sweeps at a selected angle of attack. The aerodynamic coefficients were computed in a vertical plane axis system ${ }^{7,8}$. The reshaping of the LH2 feedline fairing on the ADAC-2B results in larger peak rolling moment coefficients compared to the ADAC-2A. The ADAC-2B was tested with and without the LH2 feedline fairing to obtain aerodynamic coefficient increments. The experimental rolling moment coefficient increments compare very well with the predicted increments using the code USM3D ${ }^{4}$ as shown in fig. 48 and fig. 49, which correspond to angle of attack and roll angle sweeps at a selected supersonic Mach number in UPWT T. S. 1. The favorable wind tunnel-to-CFD comparisons supported the use of CFD as a primary tool to assess design changes to mitigate the peak rolling moments. The results of this CFD design study led to a proposed roll-control strake, which is discussed in the next section.

\section{E. Ares I ADAC-3 Force and Moment Data}

This section highlights the results obtained on the 0.01-scale Ares I ADAC-3 force and moment model with emphasis on the aerodynamic effects caused by changes to the BDM layout and the addition of a single roll control strake on the IS. An assessment of longer-term data repeatability was also obtained using the model. Schematics of the ADAC-3 and ADAC-2B configurations are presented in fig. 50. An assembly drawing of the 0.01-scale ADAC3 force and moment model is also shown in fig. 50. The Ares I ADAC-3 configuration featured ten BDM covers in an aft position on the SRB aft skirt compared to the eight BDM covers in a mid-position on the ADAC-2B. Closeup photographs of the BDMs on the ADAC-2B and ADAC-3 are shown in fig. 51. This configuration change contributed to higher axial force coefficients on the ADAC-3 compared to ADAC-2B as shown in fig. 52, which compares the longitudinal aerodynamic characteristics obtained on both models in UPWT T. S. 1 at a selected supersonic Mach number. CFD predictions for both configurations are also shown in fig. 52. The CFD method underpredicts the axial force coefficient on each configuration. However, both experiment and CFD reveal an axial force coefficient increase on the ADAC-3 compared to the ADAC-2B.

An alternate aft skirt was designed and fabricated with the ten BDM covers shifted to a mid-position on the aft skirt. Close-up photographs of the baseline aft skirt and the alternate aft skirt with BDMs shifted forward are shown in fig. 53. The alternate aft skirt promotes a significant decrease in the axial force coefficient as shown in fig. 54. This trend was observed throughout the range of Mach number tested in UPWT T. S. 1 and T. S. 2.

fig. 55 shows computer-rendered details and fig. 56 presents a close-up photograph of the single roll-control strake installed on the IS at the 270-degree azimuth position. The strake design and location were established using the code USM3D ${ }^{4}$ to counter the rolling moments caused by the asymmetric protuberances on the Ares I vehicle. The large LH2 feedline fairing positioned at the 90-degree azimuth on the IS was a primary contributor to the asymmetric rolling moments as discussed in Section IV-D on the ADAC-2B. The lateral-directional aerodynamic characteristics obtained with and without the strake in a roll sweep at a selected angle of attack in UPWT T. S. 1 are presented in fig. 57. The aerodynamic coefficients were computed in a vertical plane axis system ${ }^{7,8}$. The corresponding lateral-directional aerodynamic coefficient increments due to the strake obtained in the experiment and from CFD predictions are compared in fig. 58. Tunnel-to-tunnel comparisons of the strake aerodynamic coefficient increments obtained in roll sweeps in UPWT T. S. 1 and in the Boeing PSWT using a second ADAC-3 
model are presented in fig. 59. The results in fig. 57 to fig. 59 confirm the effectiveness of the strake in mitigating the peak asymmetric rolling moments that occur on the baseline model. The results provide additional confidence in using CFD methods as a design tool.

Within-run-series data repeatability was assessed at all Mach numbers for the 0.01-scale ADAC-3 model. The repeat runs consisted of pitch sweeps at a roll angle of 0 degrees and roll sweeps at a selected angle of attack. The initial and repeat runs were separated by other pitch and/or roll sweeps at the same Mach number. As a more rigorous assessment of data repeatability, longer-term repeat runs were performed on the baseline configuration. Specifically, additional repeat pitch and roll sweeps were obtained in T. S. 1 after completing all runs at the higher supersonic Mach numbers in T. S. 2. Consequently, three runs are displayed in the data plots consisting of two within-run series repeats and a third run obtained after removing the model from T. S. 1, performing numerous model changes and re-applications of the transition grit in T. S. 2, and re-installing the model and re-applying the transition grit in T. S. 1. Figure 60 shows the six-component aerodynamic force and moment coefficients and the corresponding delta coefficient values obtained in repeat angle of sweeps at a selected supersonic Mach number. The delta coefficient values provide a more quantitative assessment of the data repeatability by comparing the results to the $95 \%$ confidence limits for the balance accuracies expressed as aerodynamic coefficients. The delta coefficients were obtained by interpolating in each angle of attack sweep to the nominal values of the independent variable, then averaging and subtracting the averages from the interpolated data. The data variability is increased with the inclusion of the third repeat run. The delta coefficient plots indicate that the overall scatter in the normal force, axial force, and side force coefficient data is frequently greater than the upper and lower limits defined by the balance accuracies, although the data scatter is generally bounded by modified upper and lower bounds corresponding to balance accuracies of approximately $0.4 \%$ full-scale (F. S.) output or less. This corresponds to 8 to 10 times the quoted balance accuracies. The scatter in the rolling moment coefficient was within the bounds of the balance calibration accuracies. It is noted, however, that the design loads of the balances used in all of the Ares I model testing in UPWT resulted in the rolling moment gage being the least sensitive of the six force and moment components. Consequently, the 95\% confidence limits for the balance calibration accuracies on rolling moment were broader. The largest data scatter occurs in the pitching moment and yawing moment components, with the maximum data scatter approaching equivalent balance accuracies of $2 \%$ F. S. to 3\% F. S. or 60 to 100 times the quoted balance accuracies. Balance and model dynamics at the higher angles of attack in combination with the large transfer distance from the balance moment center (BMC) to the MRC at the gimble point contribute to relatively large pitching moment and yawing moment coefficient scatter bounds. Other potential factors contributing to the data scatter include non-repeatability of the transition grit application, small differences in the installation of the numerous protuberances on the model, and variations in the installation of the model chamber pressure tube bundle, which was routed externally along the sting and roll coupling for all testing in UPWT T. S. 1 and T. S. 2. It is noted, however, that similar scatter bounds were often observed in tunnel-to-tunnel data repeatability analyses because of differences in the dynamic testing environment encountered in the NASA LaRC UPWT and Boeing PSWT facilities. The observed data scatter increases the uncertainty of the supersonic aerodynamic ascent aerodynamics characterization of the Ares I vehicle, although the variation in the pitching moment and yawing moment coefficients was within the existing control capability of the vehicle.

\section{Conclusion}

One percent-scale force and moment models based on the outer mold lines of the Ares I ADAC-1, ADAC-2A, ADAC-2B, and ADAC-3 crew launch vehicle and a one percent-scale pressure model based on the ADAC-1 OML were tested in the NASA Langley Research Center Unitary Plan Wind Tunnel. Representing a design evolution spanning nearly three years, experimental data generated in this test program were used to populate supersonic ascent aerodynamic databases for Ares I. The testing of the Ares I force and moment and pressure models demonstrated the challenges of small-scale model testing at Reynolds numbers less than full-scale flight conditions. Specifically, attempts to ensure fully-turbulent flow on the models using artificial surface roughness techniques yielded inconclusive results, and limited application of a surface sublimation technique to identify the boundary layer state was unsuccessful. Consequently, a default transition grit scheme was adopted throughout the test program. Despite this limitation, however, the experimental forces and moments, surface static pressure distributions, and longitudinal line load distributions generally exhibited good agreement with CFD predictions using the code USM3D through the range of Mach number tested in UPWT. The wind tunnel testing was very effective in quantifying the overall effects of the vehicle protuberances on the six-component aerodynamic characteristics and rapidly identifying individual protuberances or protuberance subsets that were significant 
contributors to the vehicle aerodynamics. A conical flare on the ADAC-1 launch abort system tower that was a proposed cover for the abort motor nozzles exhibited a bimodal aerodynamic flow phenomenon that was sensitive to the Mach number, Reynolds number, angle of attack, and axial position of the flare on the LAS tower. These results contributed to the exclusion of the flare from succeeding Ares I design analysis cycles. A simulated exhaust plume on the ADAC-1 model confirmed the potential for plume-induced flow separation during supersonic ascent. Booster deceleration motor covers located on the interstage upstream of the frustum on the ADAC-2A model promoted unsteady shock-induced flow separation and correspondingly large increments to the axial force. In addition, aerodynamic improvements on the ADAC-2A arising from a modification of the boost protective cover over the crew exploration vehicle to a prototype ogive shape were confirmed. These findings contributed to design changes on the ADAC-2B, which featured smaller, more numerous BDM covers relocated to the first stage aft skirt and an ogive BPC OML. A large liquid hydrogen feed line fairing on the upper stage of the ADAC-2B was shown to be a primary source of asymmetric rolling moments throughout the range of supersonic Mach numbers. These results augmented a CFD investigation that culminated in the design of a single roll control strake on the ADAC-3, which effectively mitigated the peak rolling moments at all supersonic Mach numbers. The axial force was sensitive to the location of the booster deceleration motor covers on the aft skirt, and the experimental results verified the aerodynamic improvements associated with a design change that featured relocating the covers to a more forward position on the skirt. The incremental aerodynamic effects of selected protuberances measured in the wind tunnel exhibited excellent agreement with CFD predictions at all supersonic Mach numbers. Good agreement was also observed in tunnel-to-tunnel comparisons of the incremental aerodynamic effects obtained in the Unitary Plan Wind Tunnel and in the Boeing Polysonic Wind Tunnel on the same model or on a second identical model. In general, near-term within-test data repeatability was acceptable. An assessment of longer-term data repeatability featuring a complete re-installation of the model in the wind tunnel and numerous intervening model configuration changes revealed larger data scatter. The data scatter was within the vehicle's control authority in all cases. Variation in the model installation process, installation of the model protuberances, routing of external instrumentation bundles, and application of boundary layer transition grit were potential sources of the increased data scatter.

\section{References}

${ }^{1}$ NASA's Exploration Systems Architecture Study, Final Report. NASA TM 2005-214062, November 2005.

${ }^{2}$ Huebner, L. D., Hall, R. M., Haynes, D. A., Pamadi, B. N., Taylor, T. L., and Seaford, C. M., "Status, Plans and Initial Results for Ares I Crew Launch Vehicle Aerodynamics,” Journal of the British Interplanetary Society, Vol. 61, 2008.

${ }^{3}$ Tomek, W. G., “Overview of Experimental Investigations for Ares Launch Vehicle Development," AIAA 49 ${ }^{\text {th }}$ Aerospace Sciences Meeting, American Institute of Aeronautics and Astronautics, Reston, VA (submitted for publication).

${ }^{4}$ Abdol-Hamid, K. S. and Ghaffari, F., “An Overview of Ares-I CFD Ascent Aerodynamic Data Development and Analysis Based on USM3D,” AIAA $49^{\text {th }}$ Aerospace Sciences Meeting, American Institute of Aeronautics and Astronautics, Reston, VA (submitted for publication).

${ }^{5}$ Erickson, G. E., "Overview of Selected Measurement Techniques for Aerodynamics Testing in the NASA Langley Unitary Plan Wind Tunnel,” AIAA 2000-32396, AIAA Fluids 2000 Conference, 19-22 June 2000.

${ }^{6}$ Schwartz, R. J. and Fleming, G. A., “Advanced Visualization of Experimental Data in Real Time Using LiveView3D," AIAA 2006-3302, 25 ${ }^{\text {th }}$ AIAA Aerodynamic Measurement Technology and Ground Testing Conference, 5-8 June 2006.

${ }^{7}$ Erickson, G. E., "0.01-Scale CLV DAC-2A Force and Moment Model Testing in the NASA Langley Research Center Unitary Plan Wind Tunnel,” Ares-AD-TA-0004, July 2007. (ITAR Restricted)

${ }^{8}$ Erickson, G. E., "0.01-Scale Ares I ADAC-3 (A106) Force and Moment Model Testing in the NASA Langley Research Center Unitary Plan Wind Tunnel,” Ares-AD-TA-0018, June 2010. (ITAR Restricted)

${ }^{9}$ Jackson, C. M., Jr., Corlett, W. A., and Monta, W. J., "Description and Calibration of the Langley Unitary Plan Wind Tunnel,” NASA TP 1905, August 1981.

${ }^{10}$ Paulson, John W., Jr., "Preliminary Analysis of Ares I Alternate Launch Abort System (ALAS) Configurations Tested in the Boeing Polysonic Wind Tunnel,” NASA/CR-2007-214877, April 2007.

${ }^{11}$ Wilcox, F. J., "Use of Colored Water Flow Visualization in a Supersonic Wind Tunnel to Investigate Cavity Flow Fields," Flow Visualization VI -- Proceedings of the Sixth International Symposium on Flow Visualization, Springer-Verlag, October 1992, pp. 41-45. 


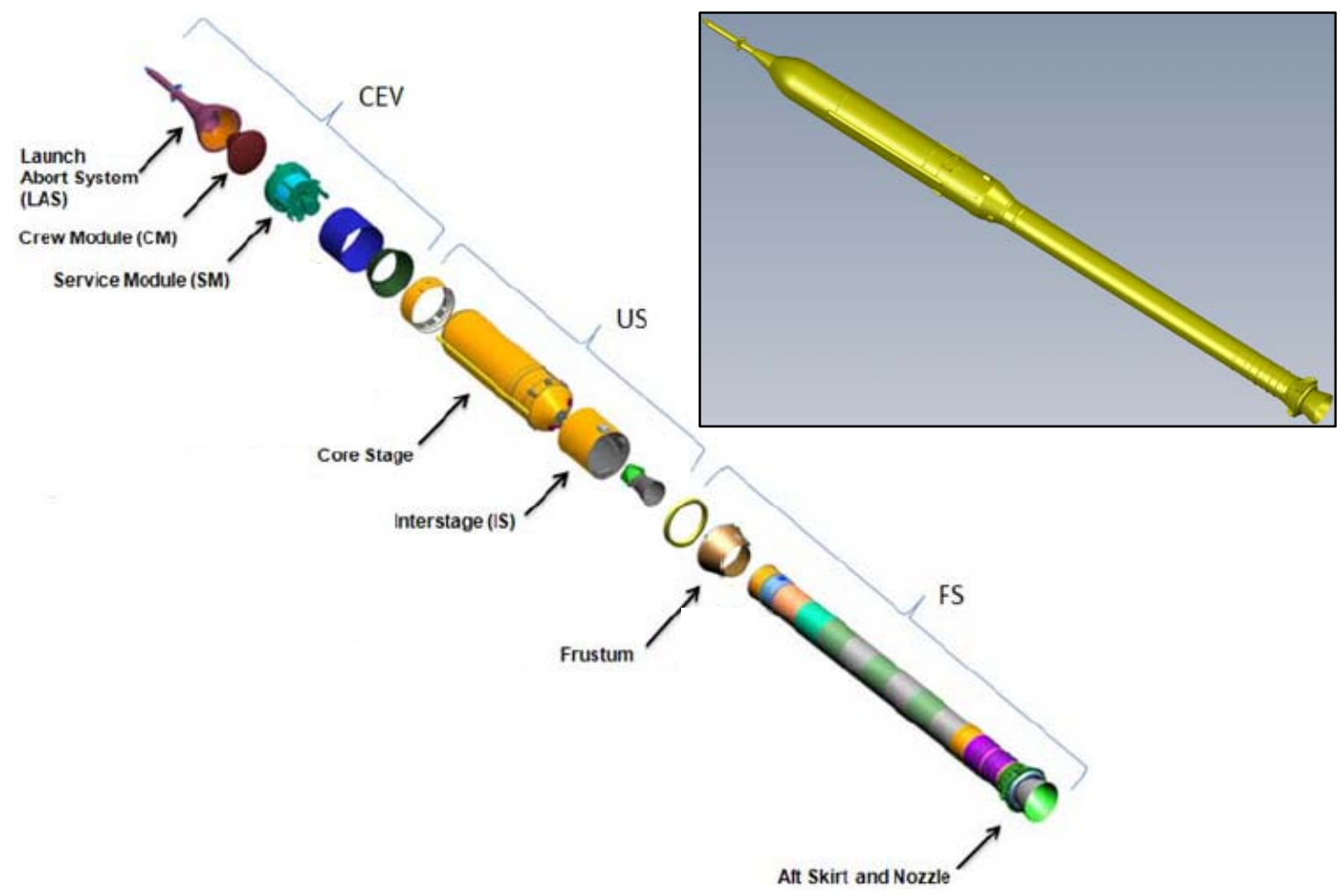

Fig. 1. Ares I integrated launch vehicle.

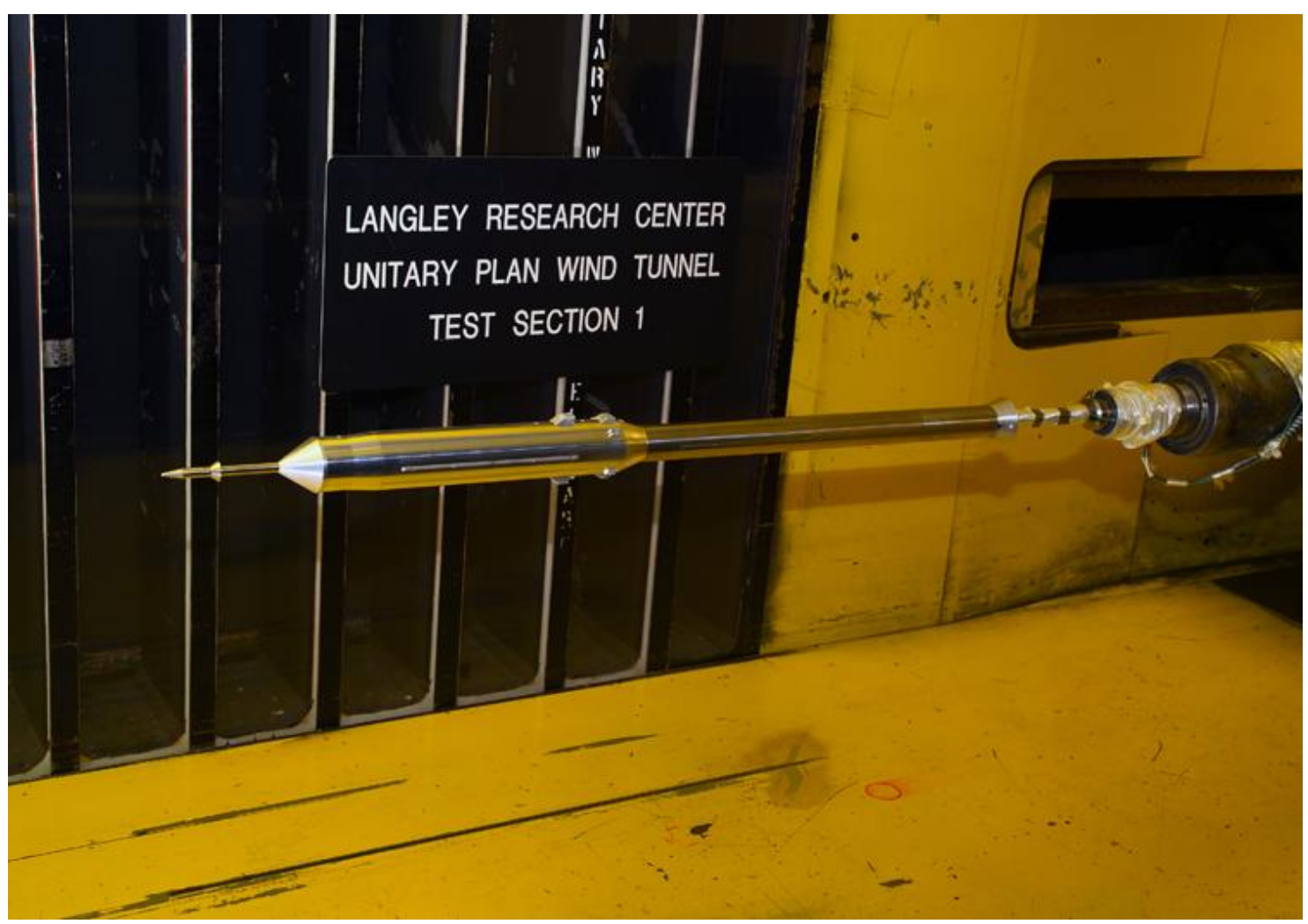

Fig. 2. Ares I ADAC-1 0.01-scale force and moment model installed in UPWT T. S. 1. 


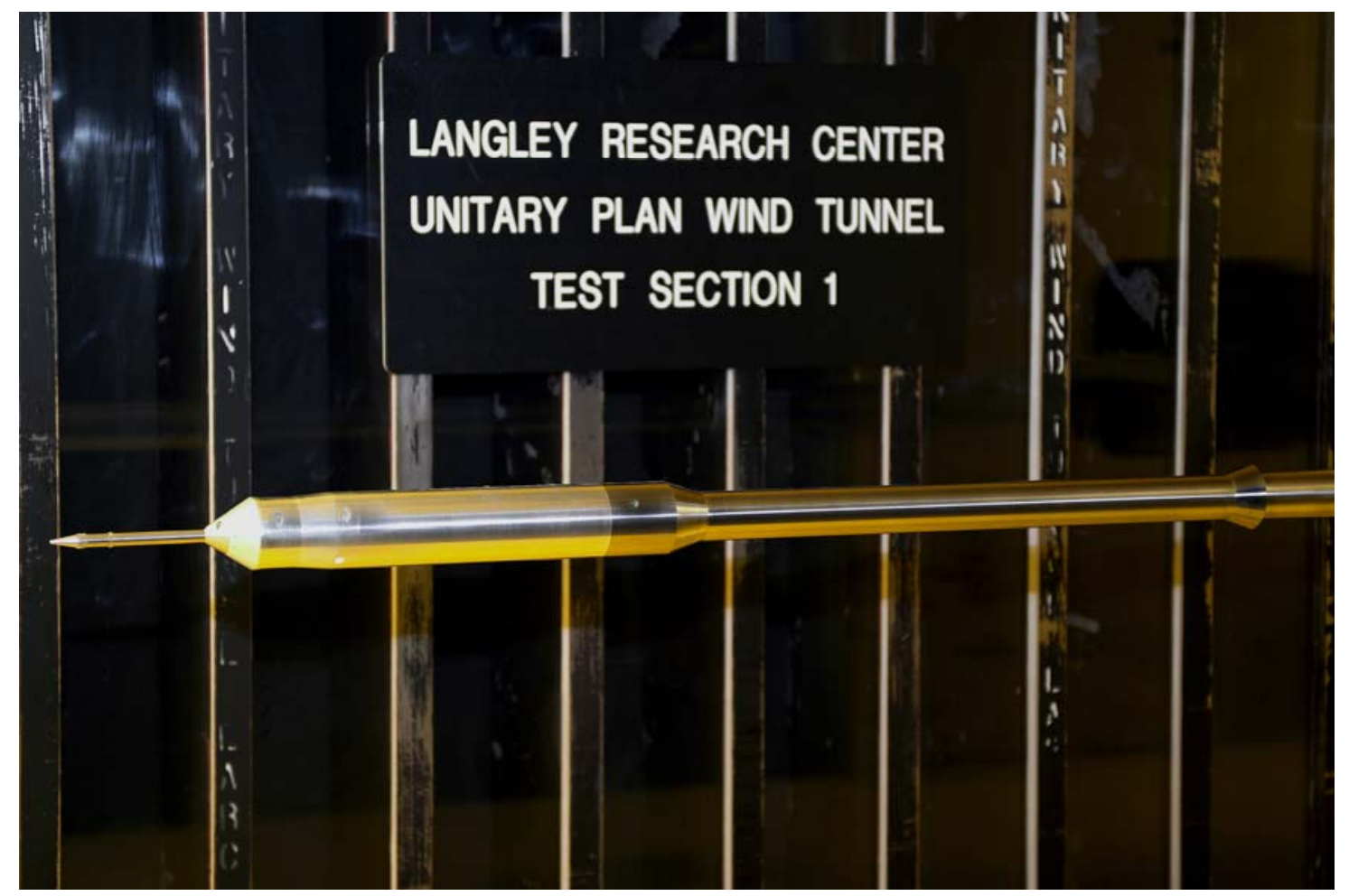

Fig. 3. Ares I ADAC-1 0.01-scale pressure model installed in UPWT T. S. 1.

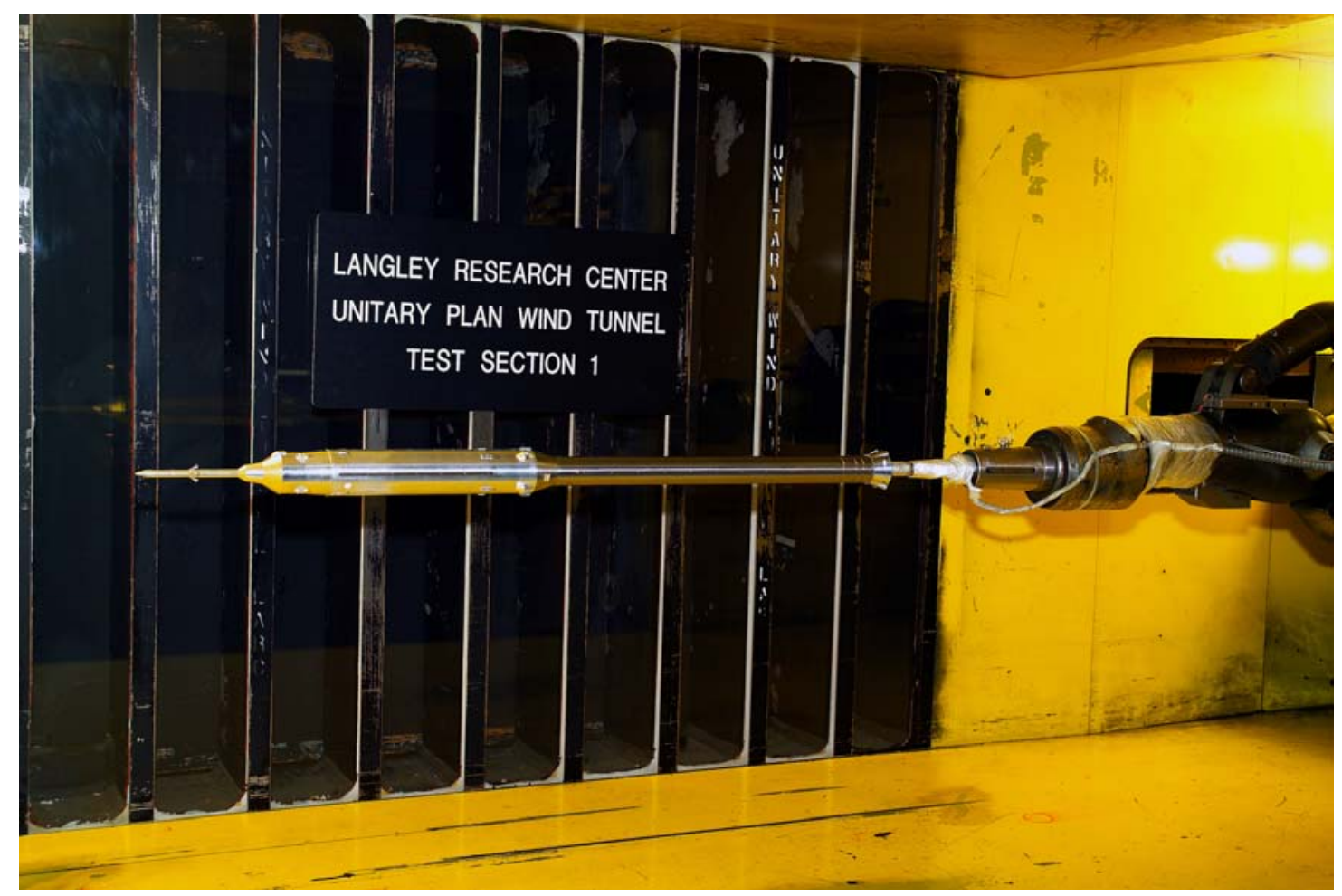

Fig. 4. Ares I ADAC-2A 0.01-scale force and moment model installed in UPWT T. S. 1. 


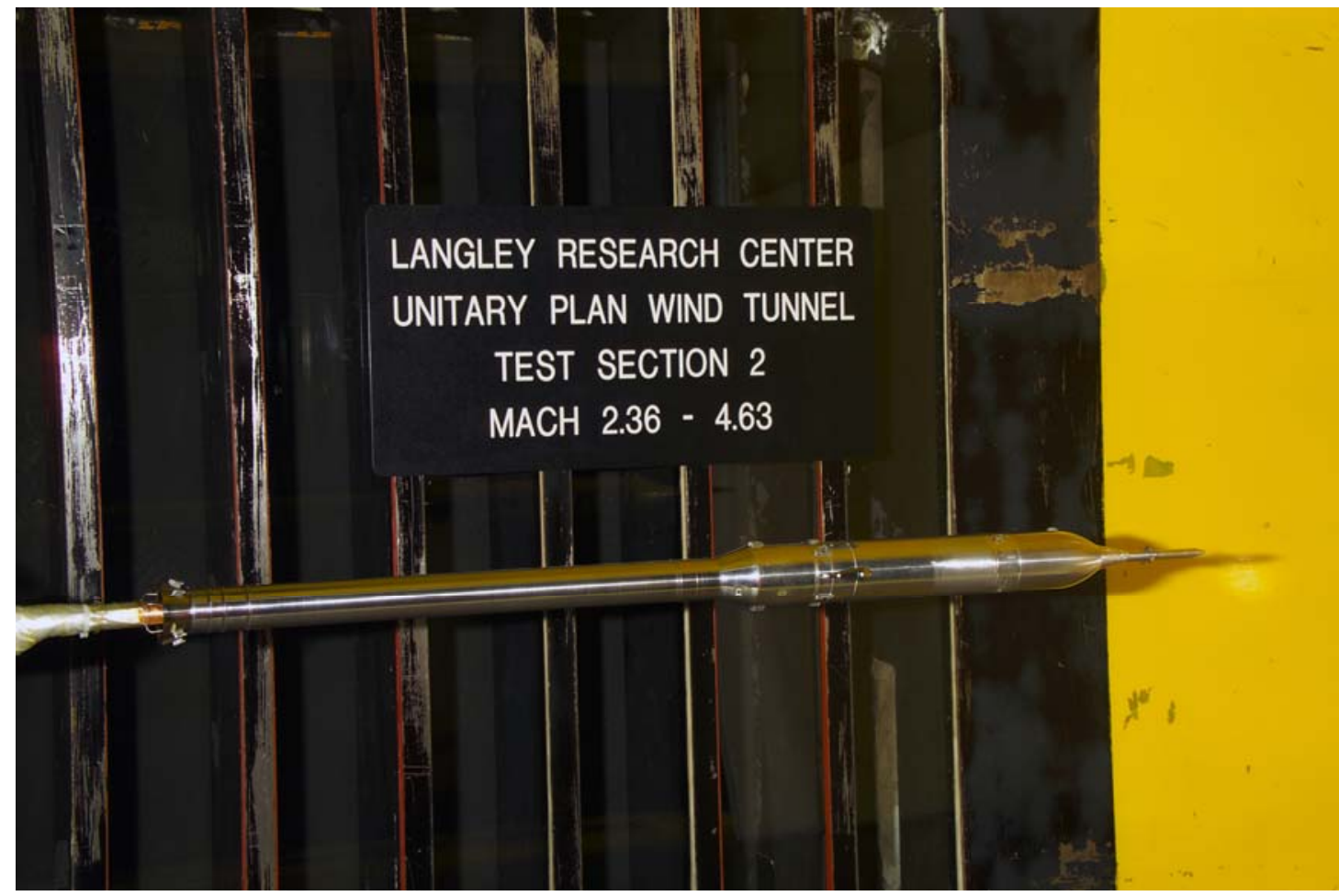

Fig. 5. Ares I ADAC-2B 0.01-scale force and moment model installed in UPWT T. S. 2.

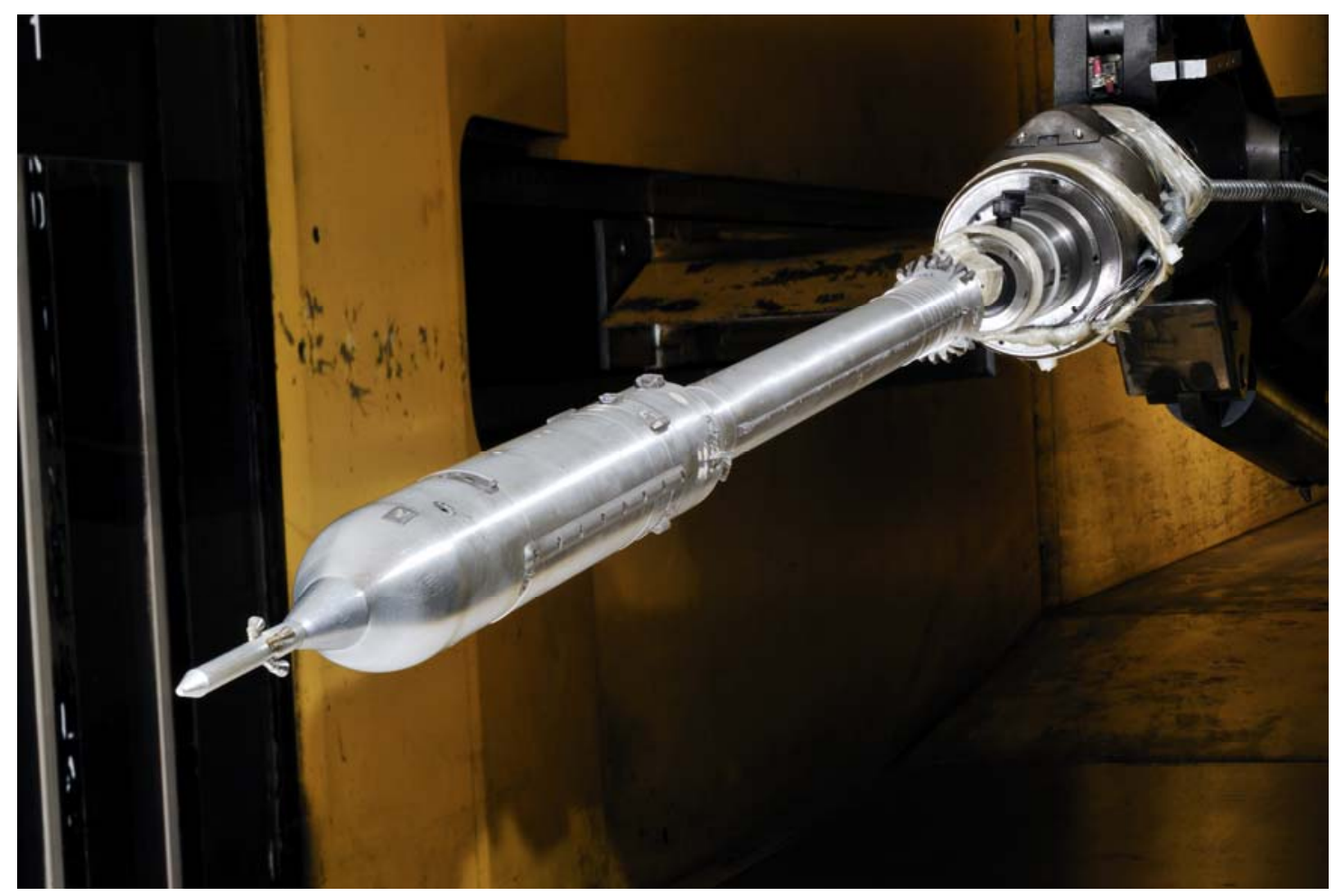

Fig. 6. Ares I ADAC-3 0.01-scale force and moment model installed in UPWT T. S. 1. 


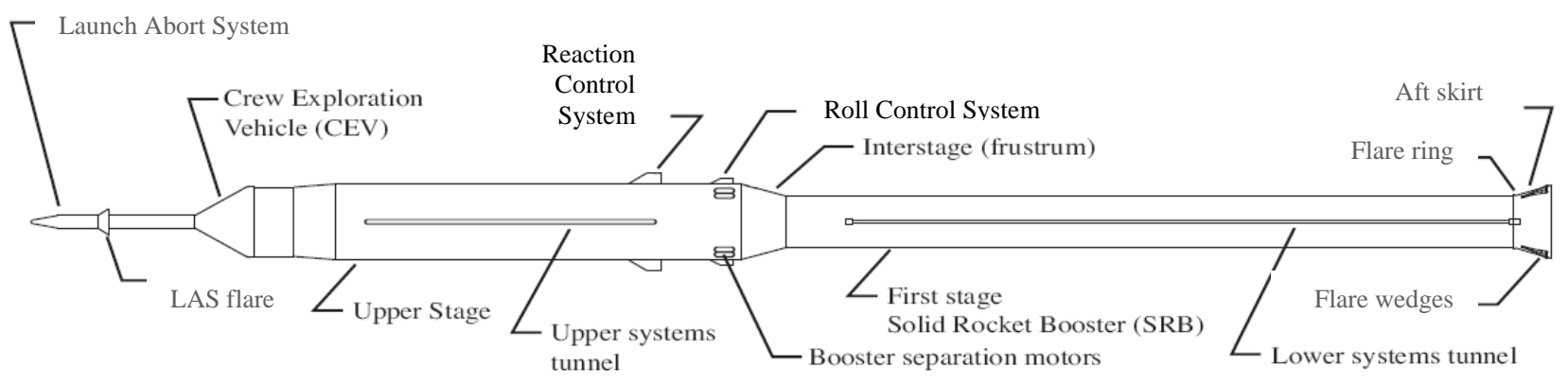

Fig.7. Sketch of Ares I ADAC-1 with protuberances.

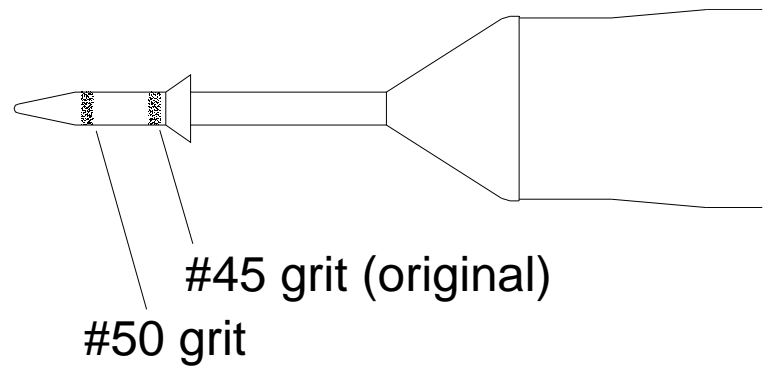

Fig. 8. Sketch of Ares I ADAC-1 model transition grit patterns used in UPWT T. S. 1.

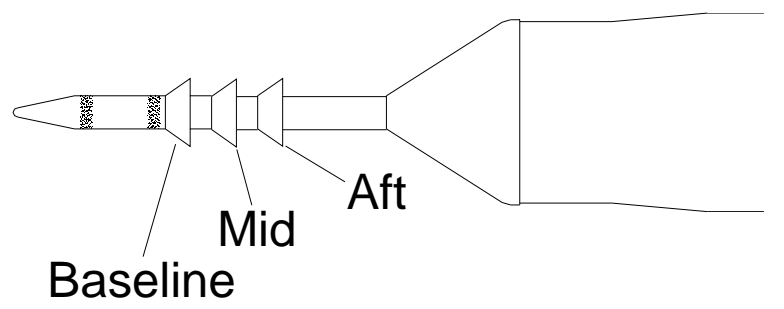

Fig. 9. Sketch of Ares I ADAC-1 model LAS flare positions. 


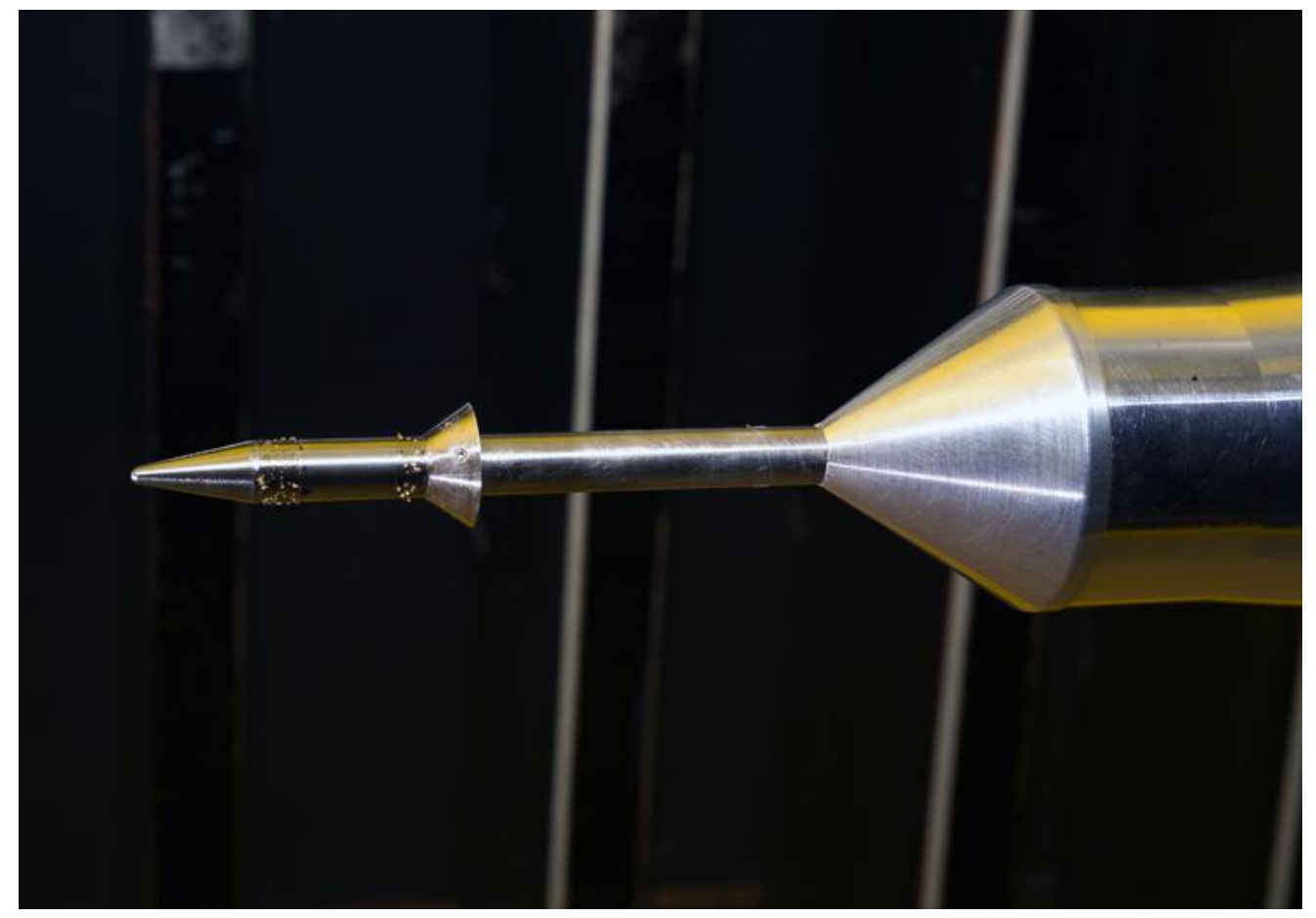

(a) LAS flare in baseline position.

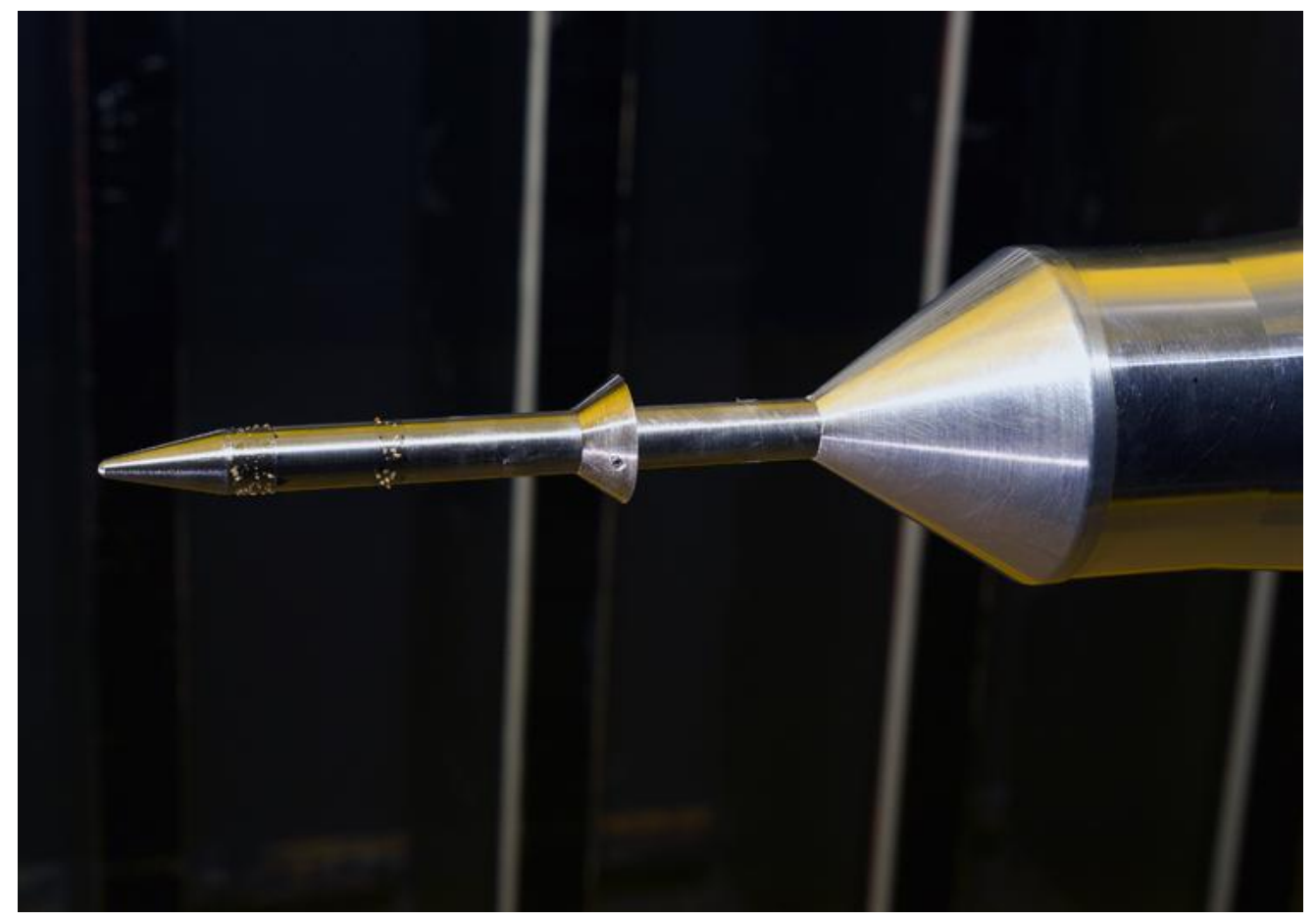

(b) LAS flare in aft position.

Fig. 10. Photographs of the 0.01-scale Ares I ADAC-1 model LAS flare in baseline and aft positions. 


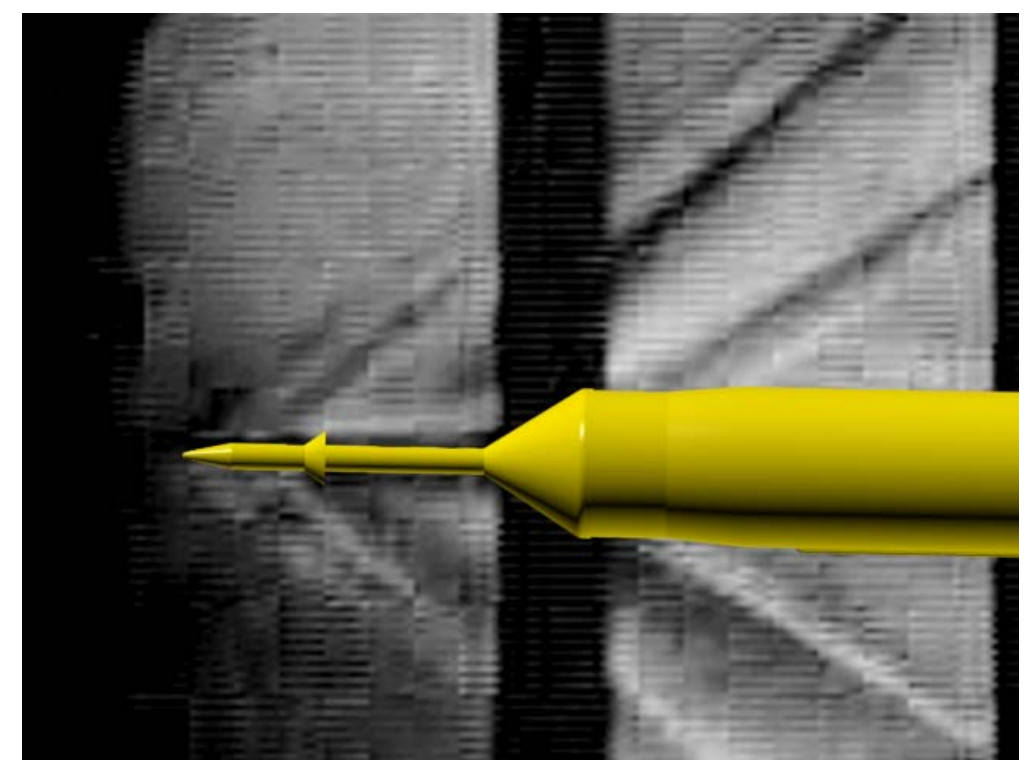

(a) "Low drag" condition

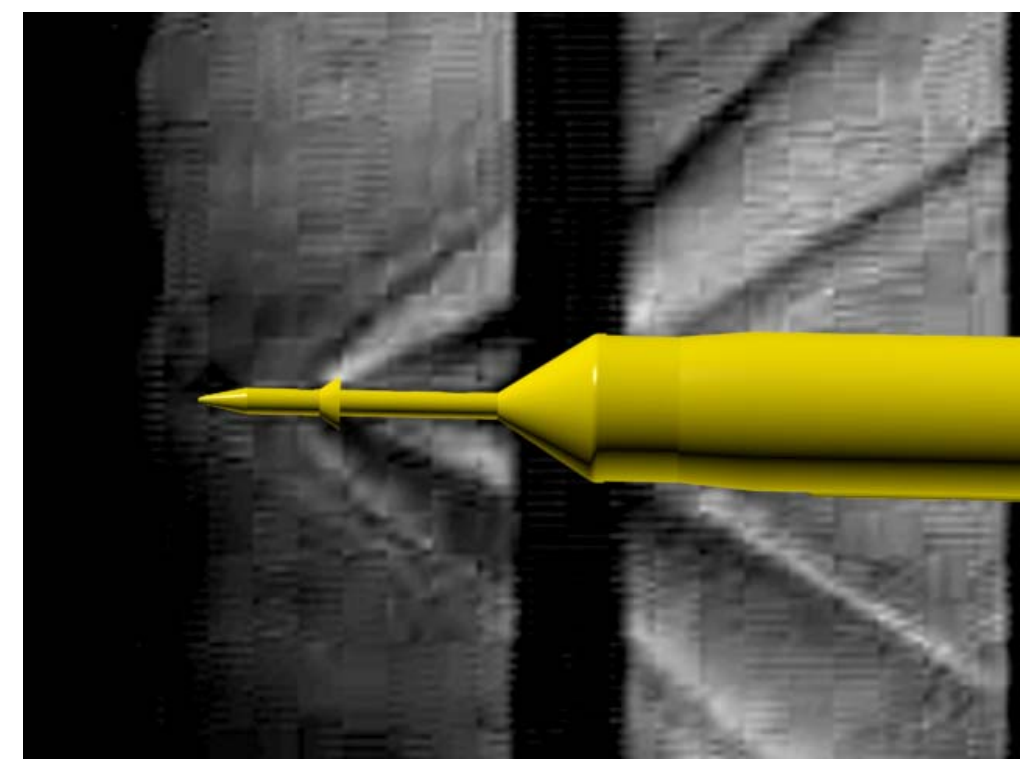

(b) “High drag” condition

Fig. 11. Schlieren video frames showing bimodal flow phenomenon with LAS flare in baseline position on the 0.01-scale Ares I ADAC-1 force and moment model in UPWT T. S. 1. 


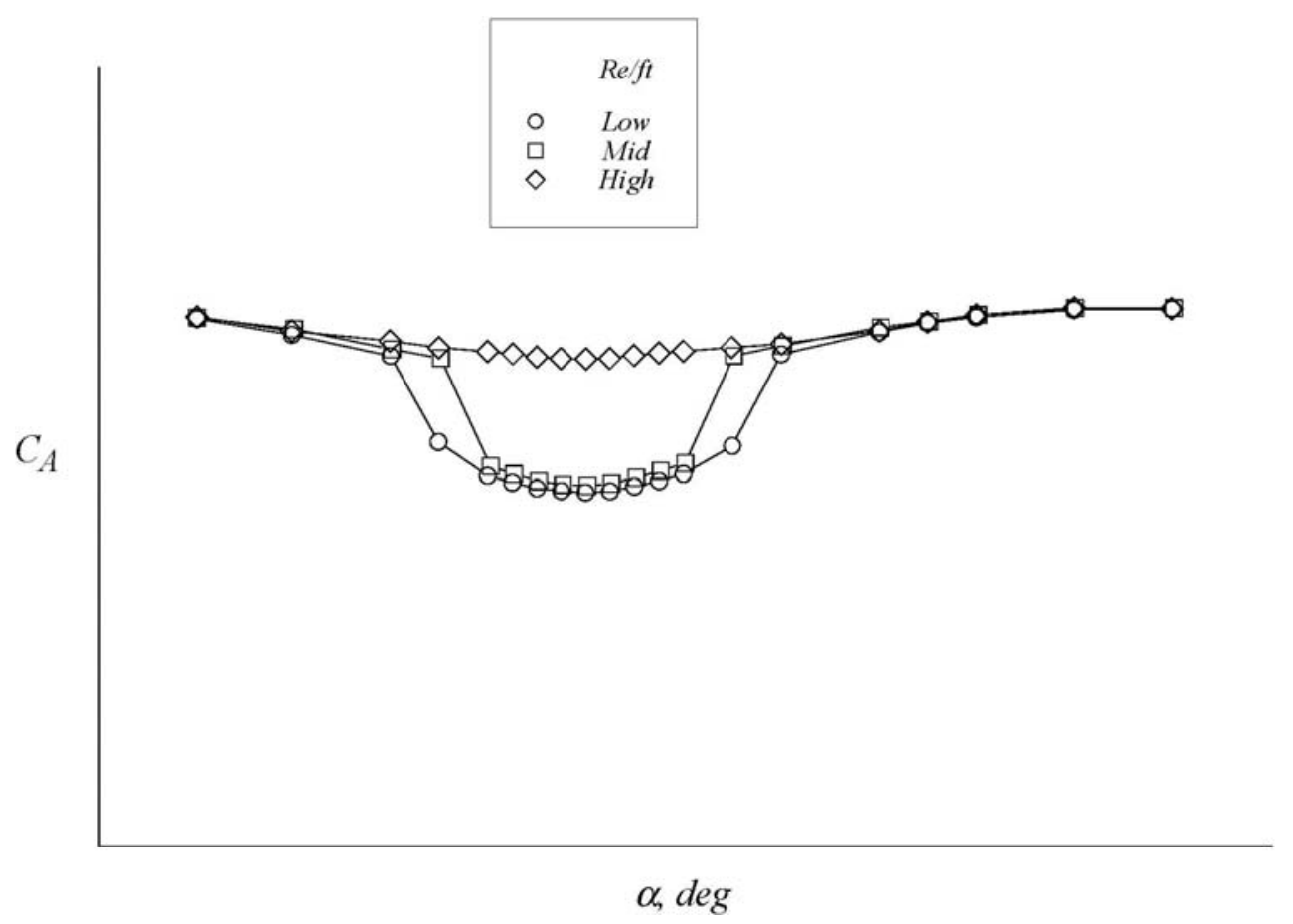

Fig. 12. Reynolds number effect on the 0.01-scale Ares I ADAC-1 model axial force coefficient with LAS flare in the baseline position at a lower supersonic Mach number in UPWT T. S. 1.

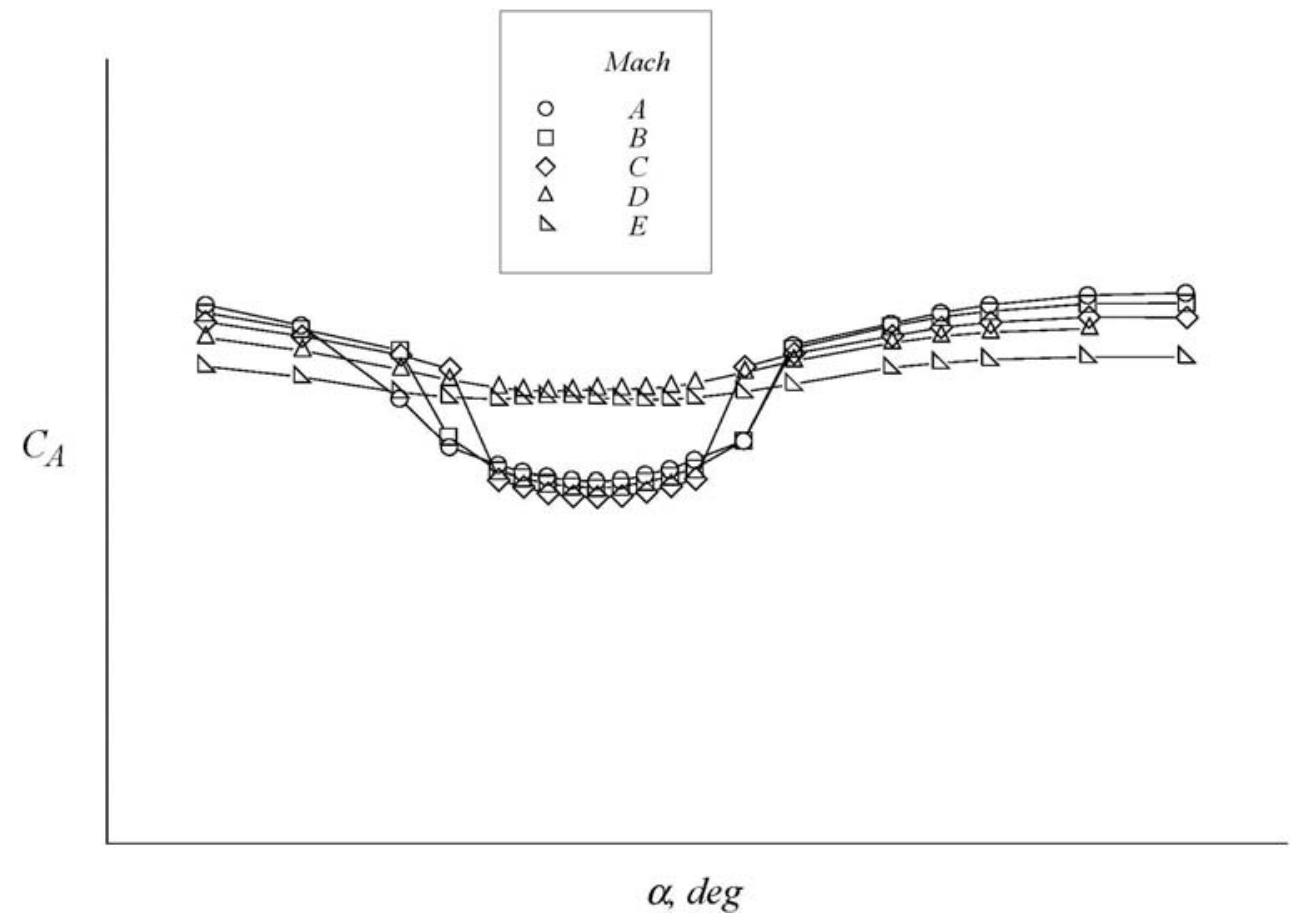

Fig. 13. Mach number effect on the 0.01-scale Ares I ADAC-1 model axial force coefficient with LAS flare in the baseline position at a lower Reynolds number in UPWT T. S. 1. 


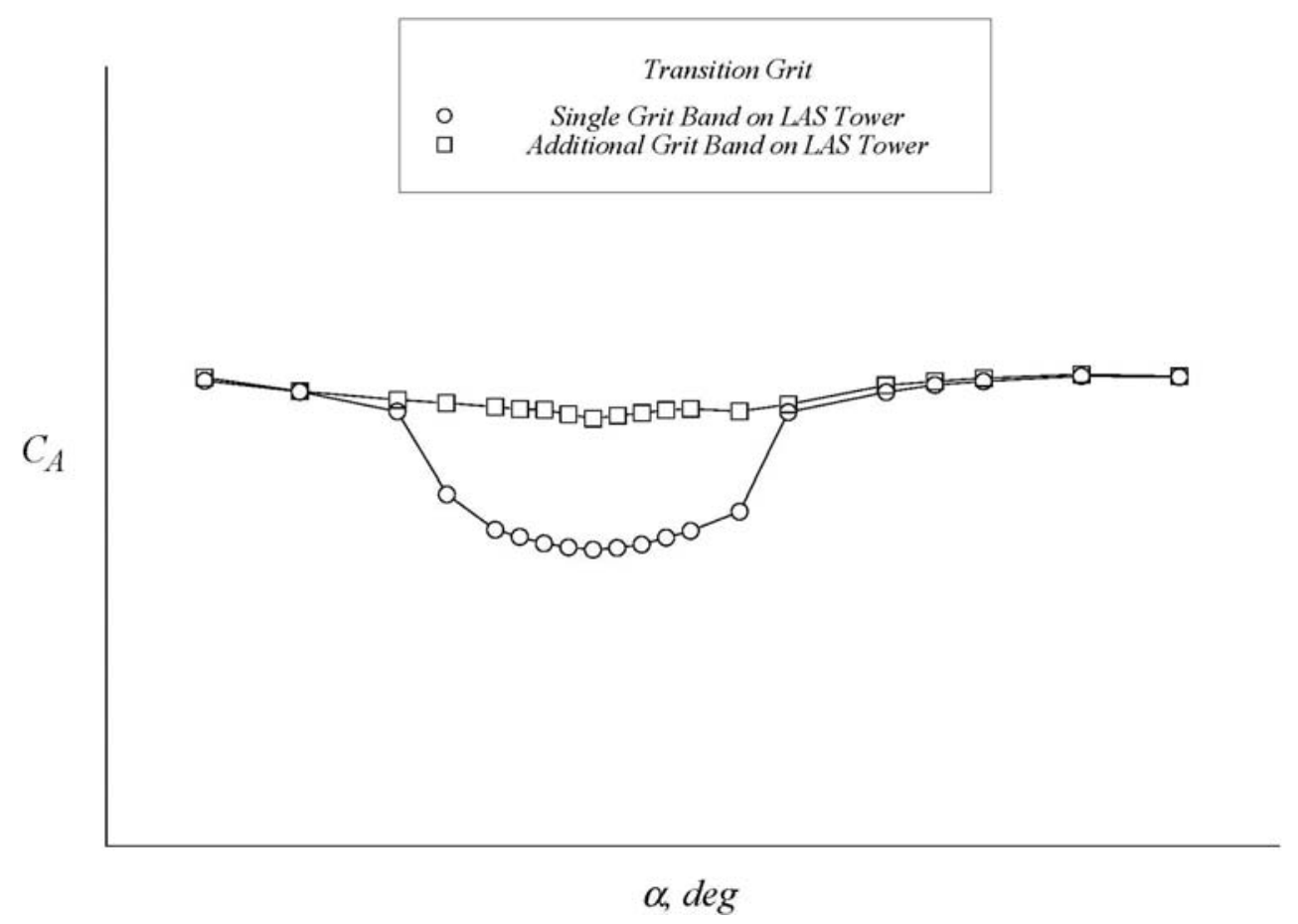

Fig. 14. Additional grit band effect on the 0.01-scale Ares I ADAC-1 model axial force coefficient with LAS flare in baseline position at lower Reynolds number and Mach number in UPWT T. S. 1.

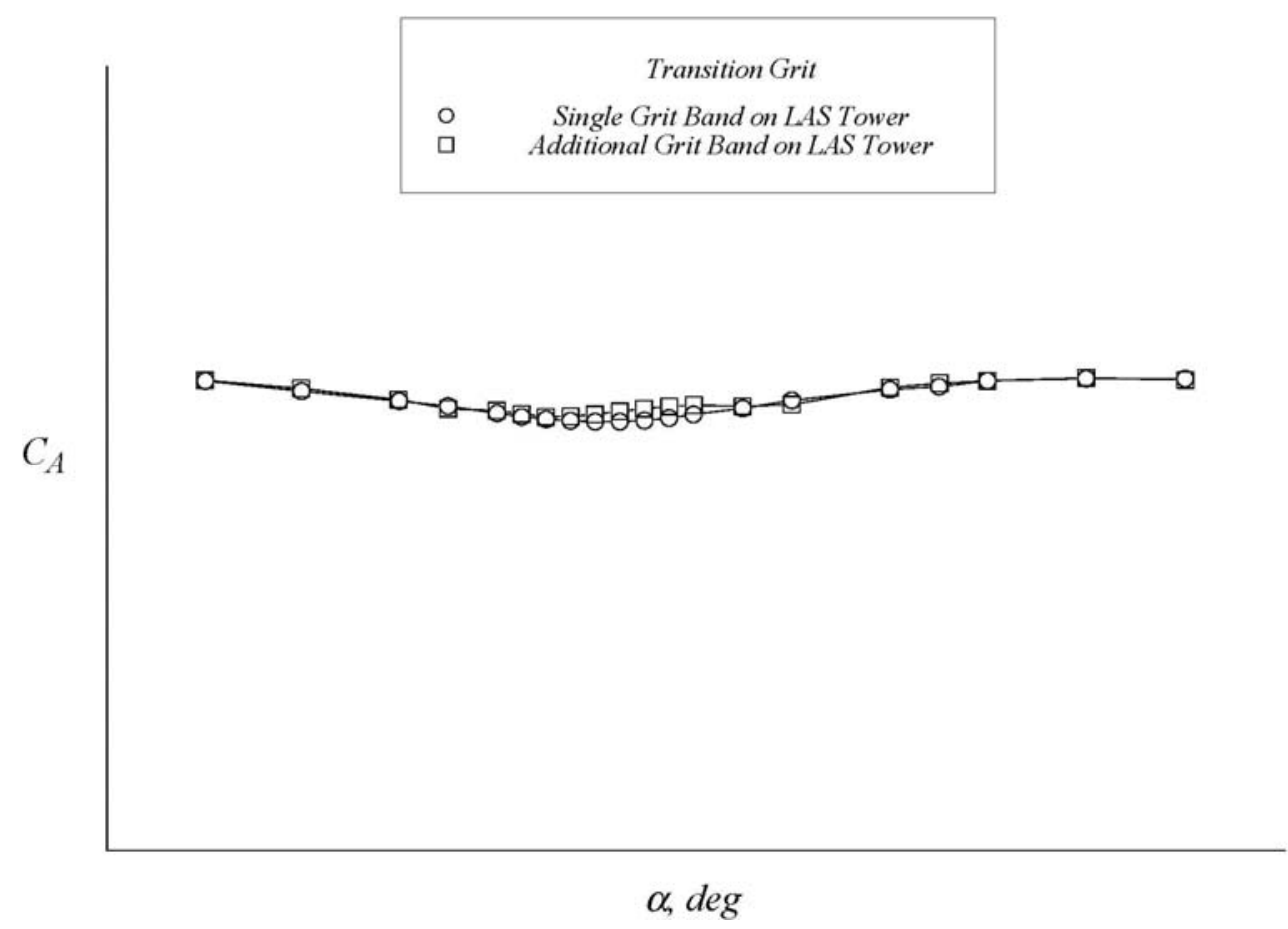

Fig. 15. Additional grit band effect on 0.01-scale Ares I ADAC-1 model axial force coefficient with LAS flare in baseline position at higher Reynolds number and selected Mach number in UPWT T. S. 1. 


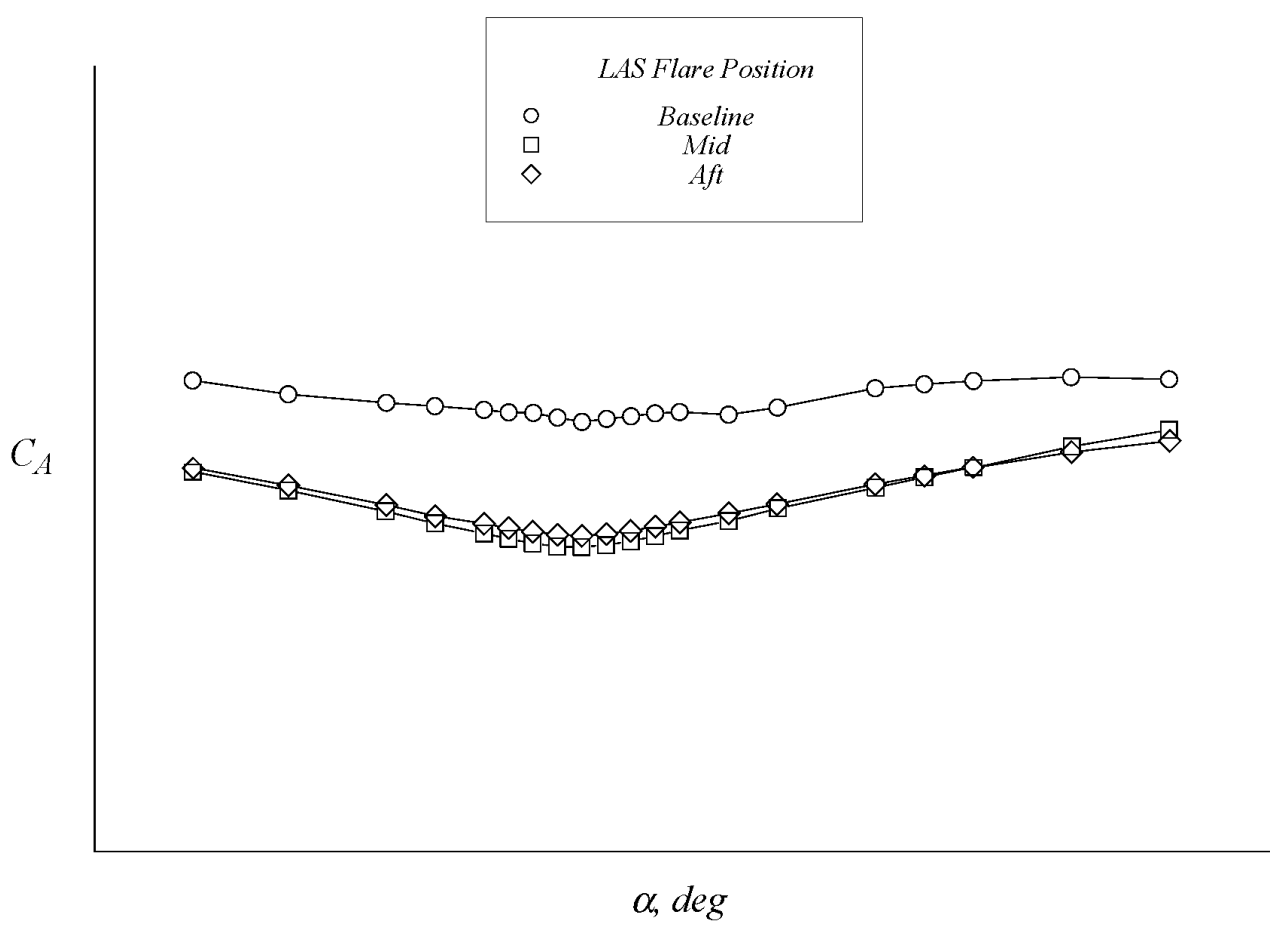

Fig. 16. LAS flare position effect on the 0.01-scale Ares I ADAC-1 axial force coefficient with additional grit band on LAS at lower Reynolds number and selected Mach number in UPWT T. S. 1.

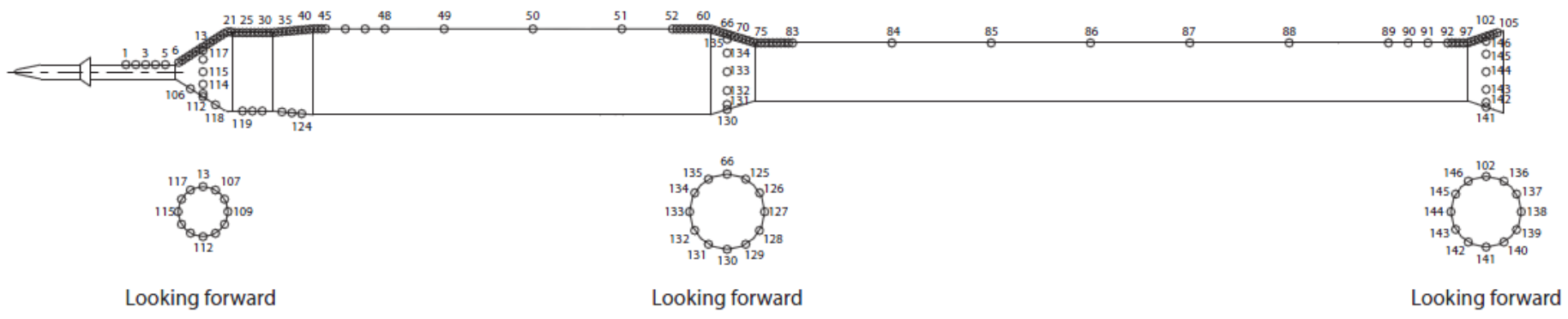

Fig. 17. Pressure tap layout on the 0.01-scale Ares I ADAC-1 wind tunnel model. 

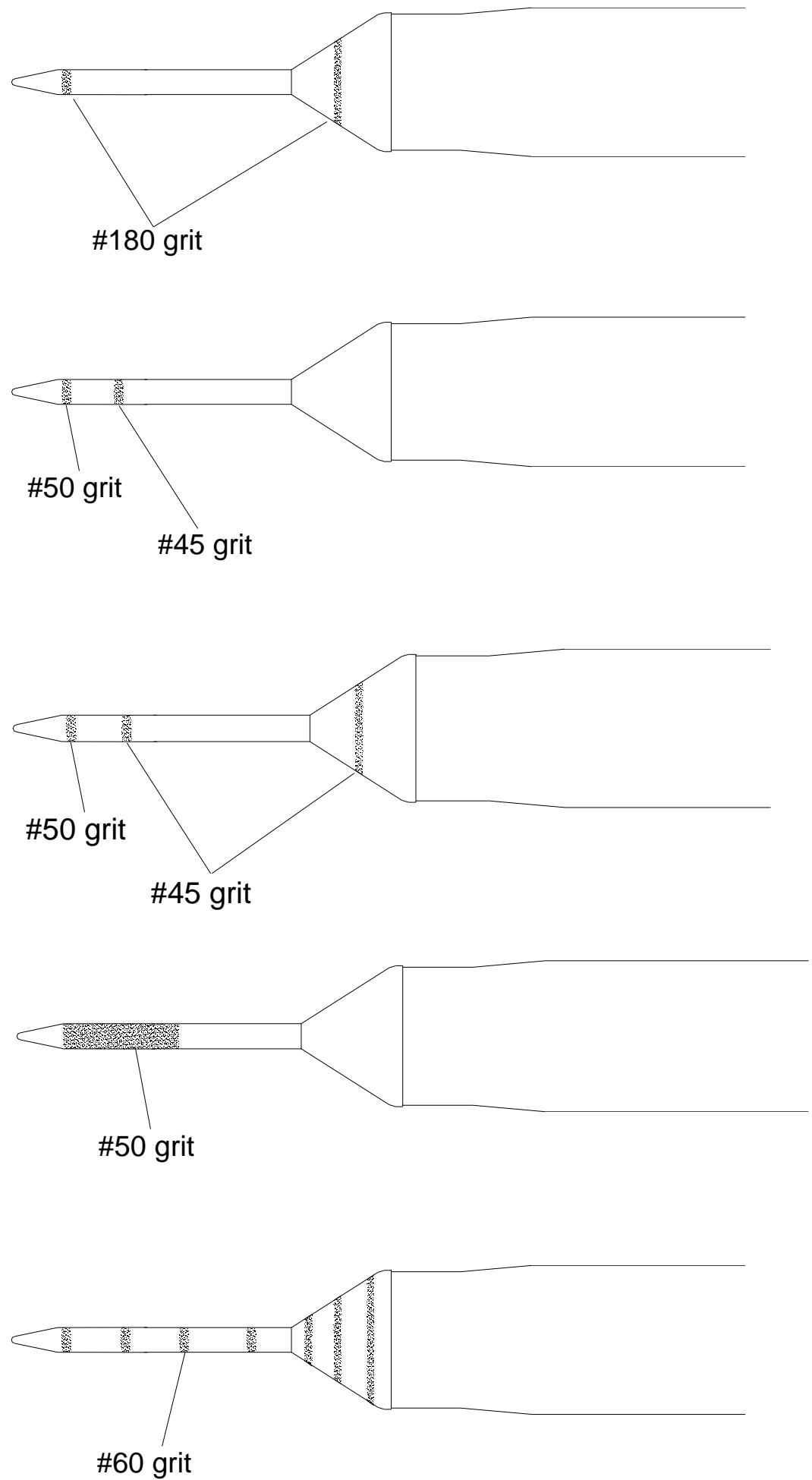

Fig. 18. Selected transition grit patterns applied to the 0.01-scale Ares I ADAC-1 pressure model in UPWT T. S. 1. 


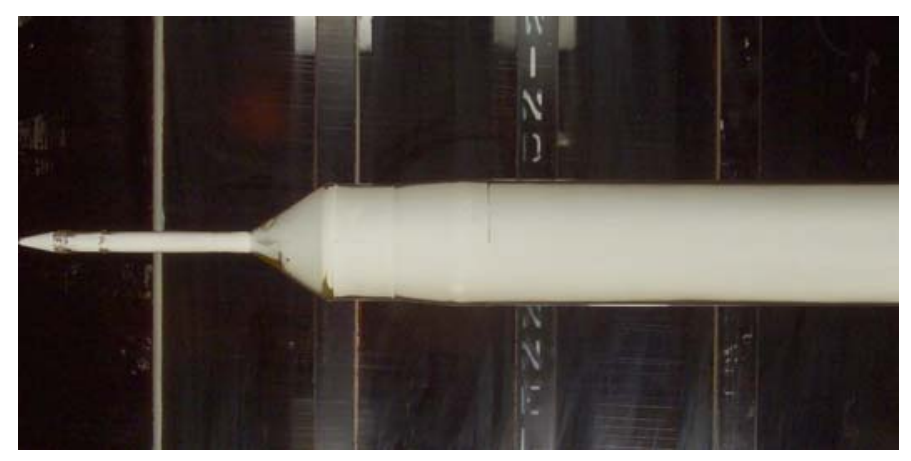

Elapsed Time $=15$ minutes

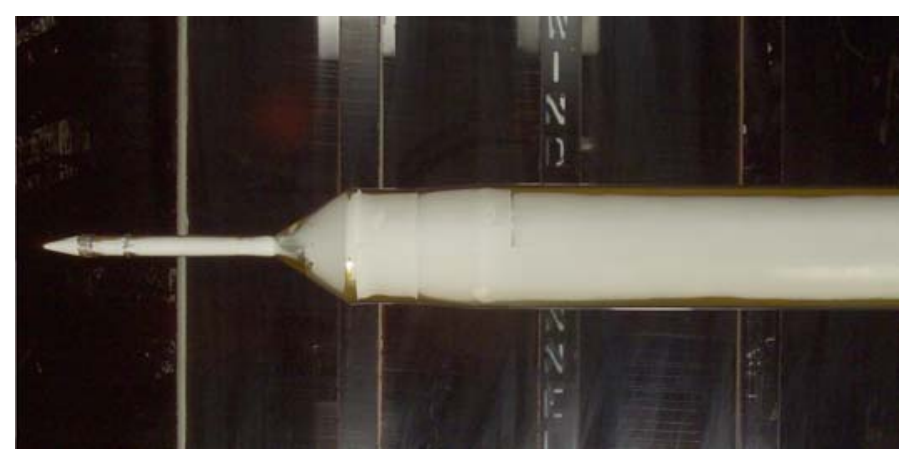

Elapsed Time $=20$ minutes

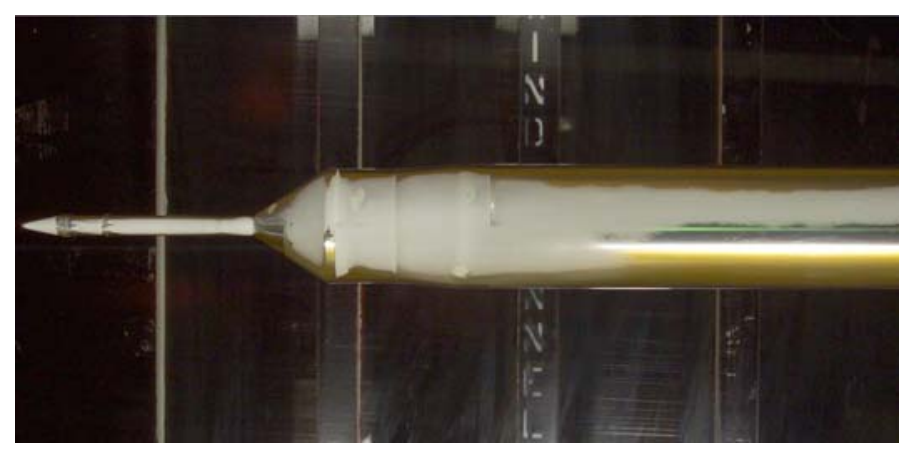

Elapsed Time $=25$ minutes

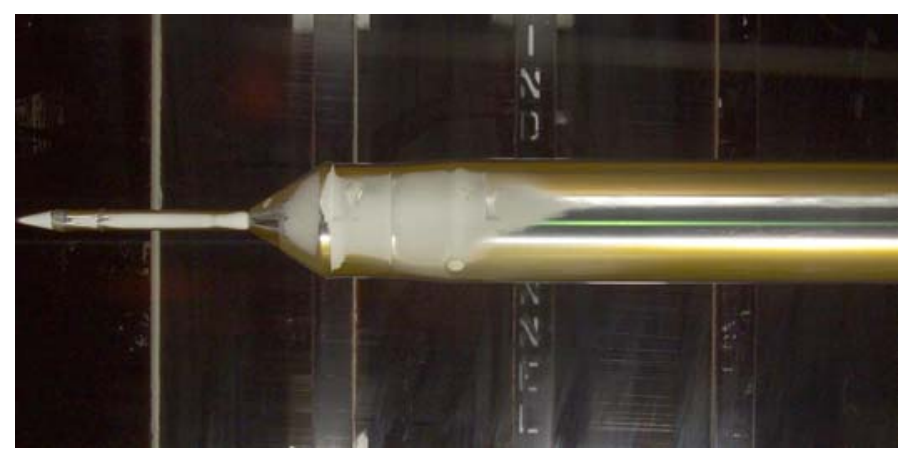

Elapsed Time $=30$ minutes

Fig. 19. Selected chemical sublimation images on the 0.01-scale Ares I ADAC-1 pressure model with \#50 and \#45 grit bands on LAS at a lower supersonic Mach number in UPWT T. S. 1. 


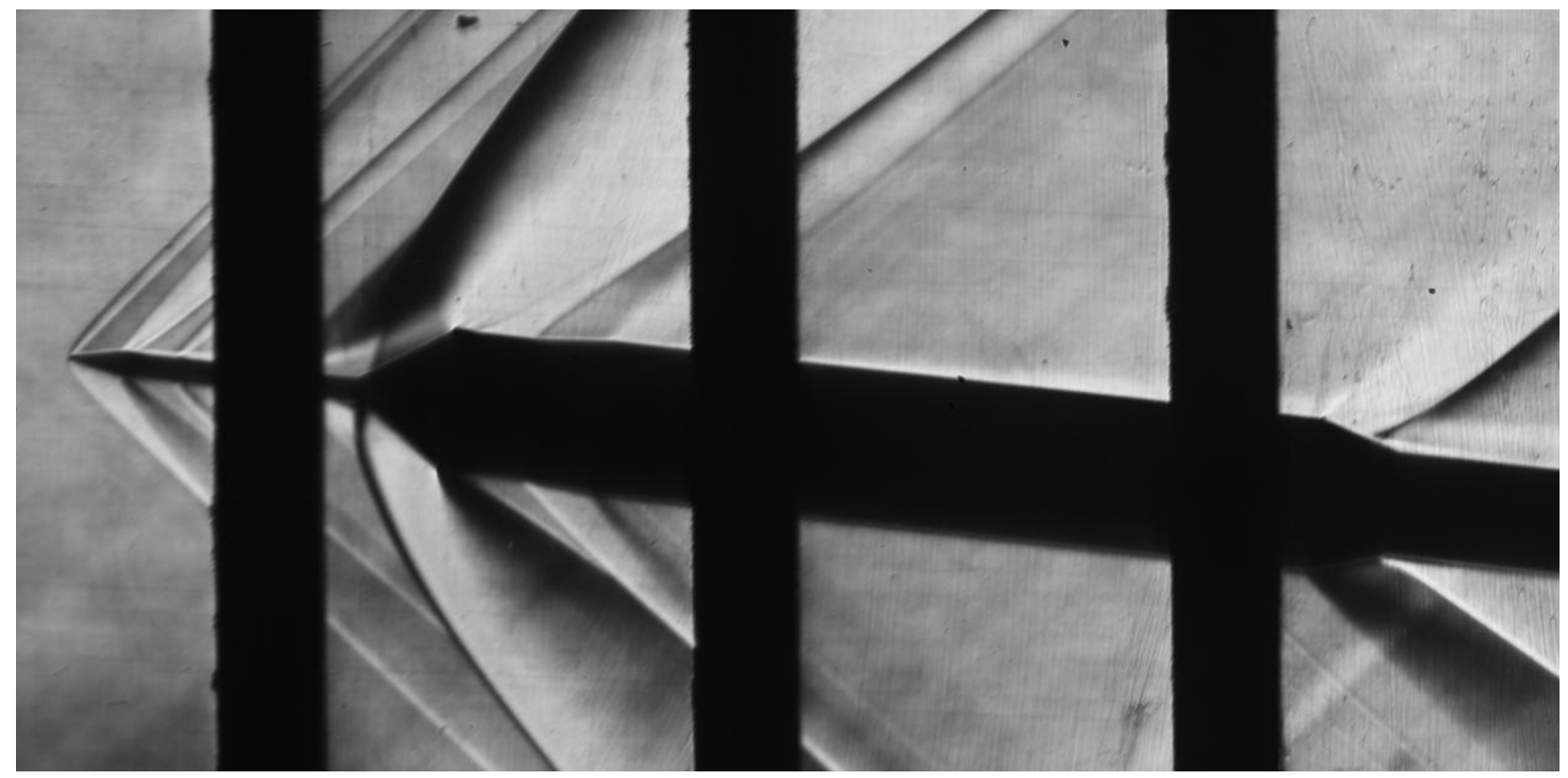

(a) Lower supersonic Mach number.

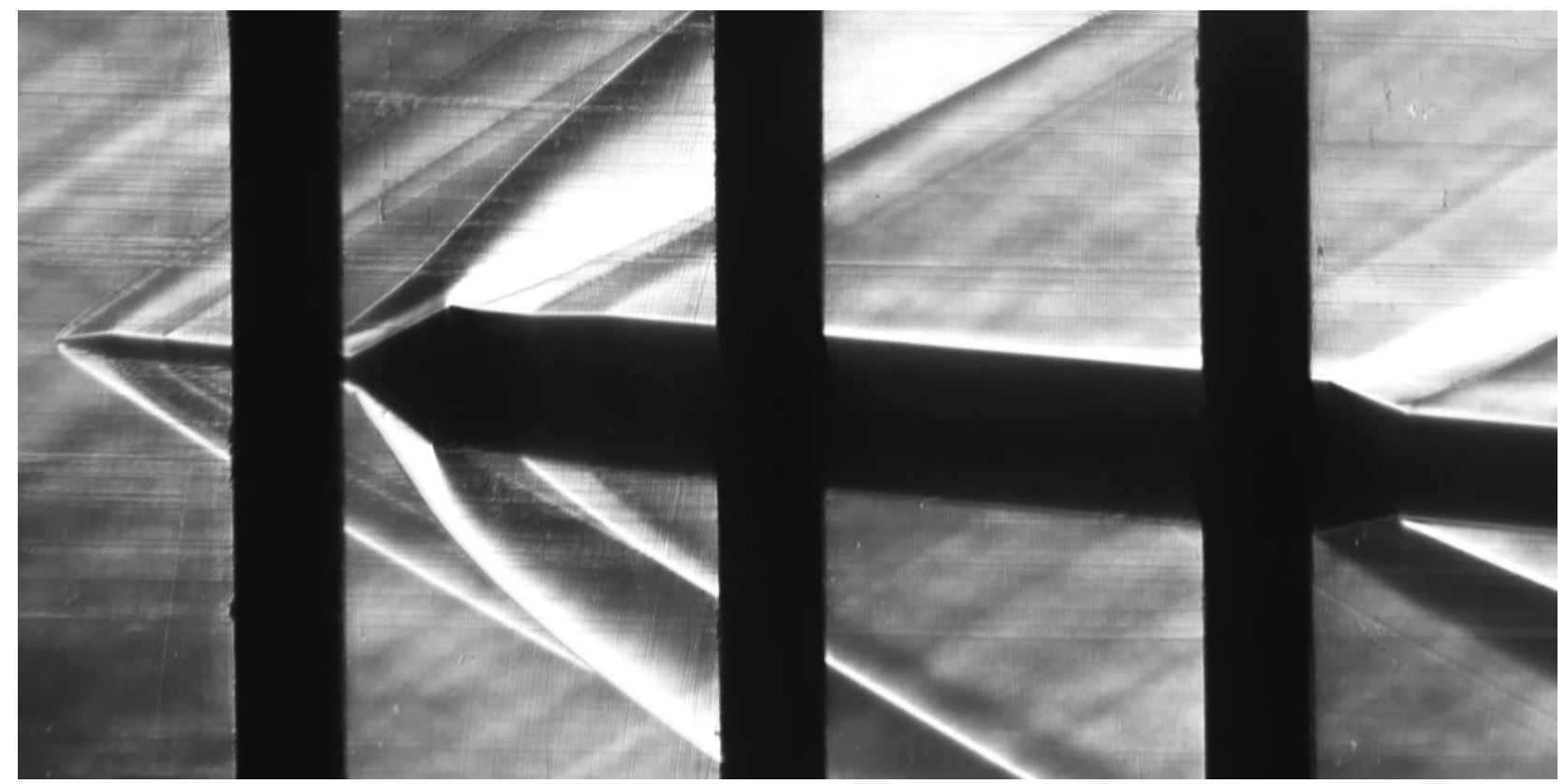

(b) Higher supersonic Mach number.

Fig. 20. Schlieren flow visualization images of the 0.01-scale Ares I ADAC-1 pressure model at two different Mach numbers in UPWT T. S. 1. 

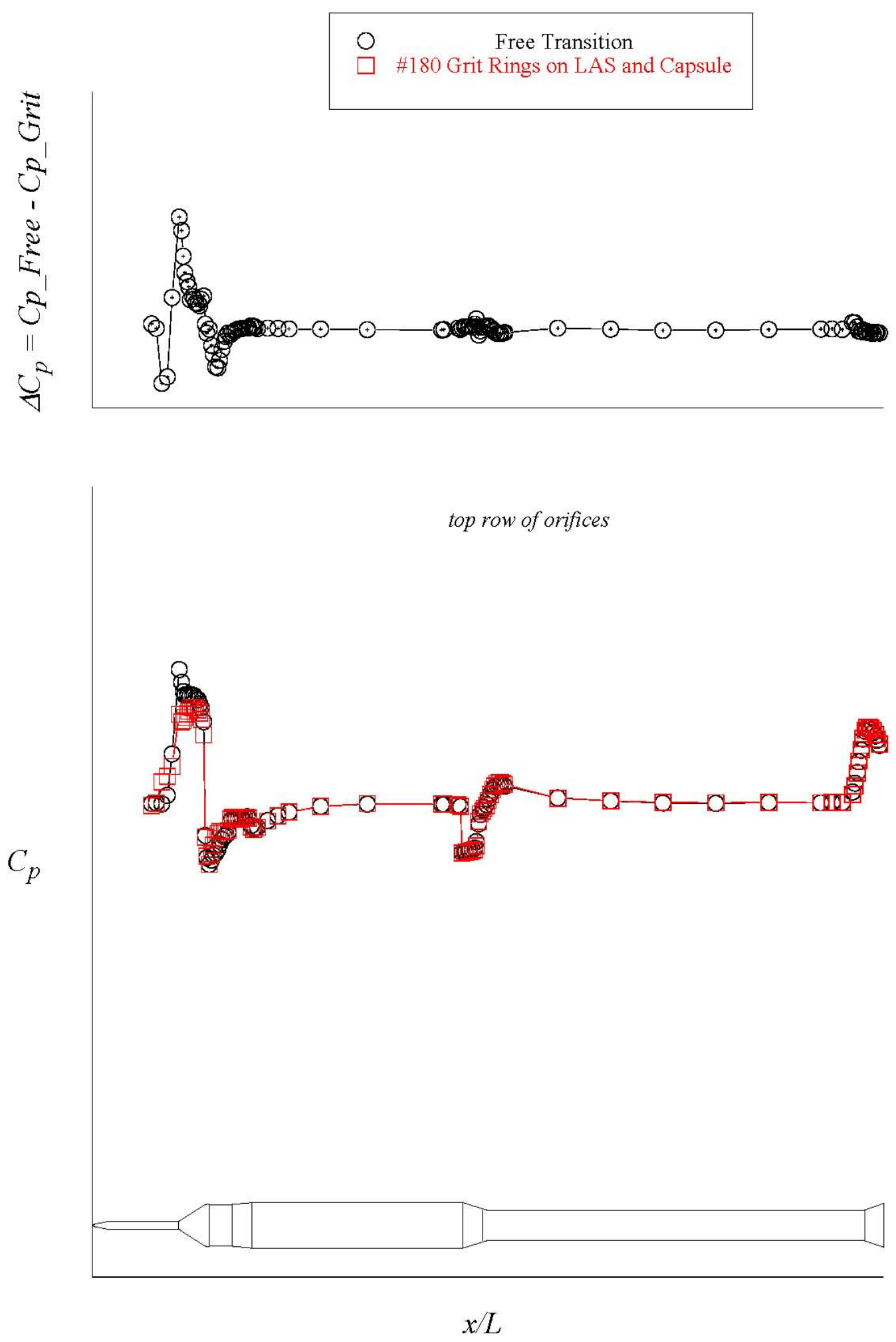

(a) \#180 grit rings on LAS and capsule.

Fig. 21. Transition grit effect on the 0.01-scale Ares I ADAC-1 model pressure distributions at a selected Mach number and angle of attack in UPWT T. S. 1. 

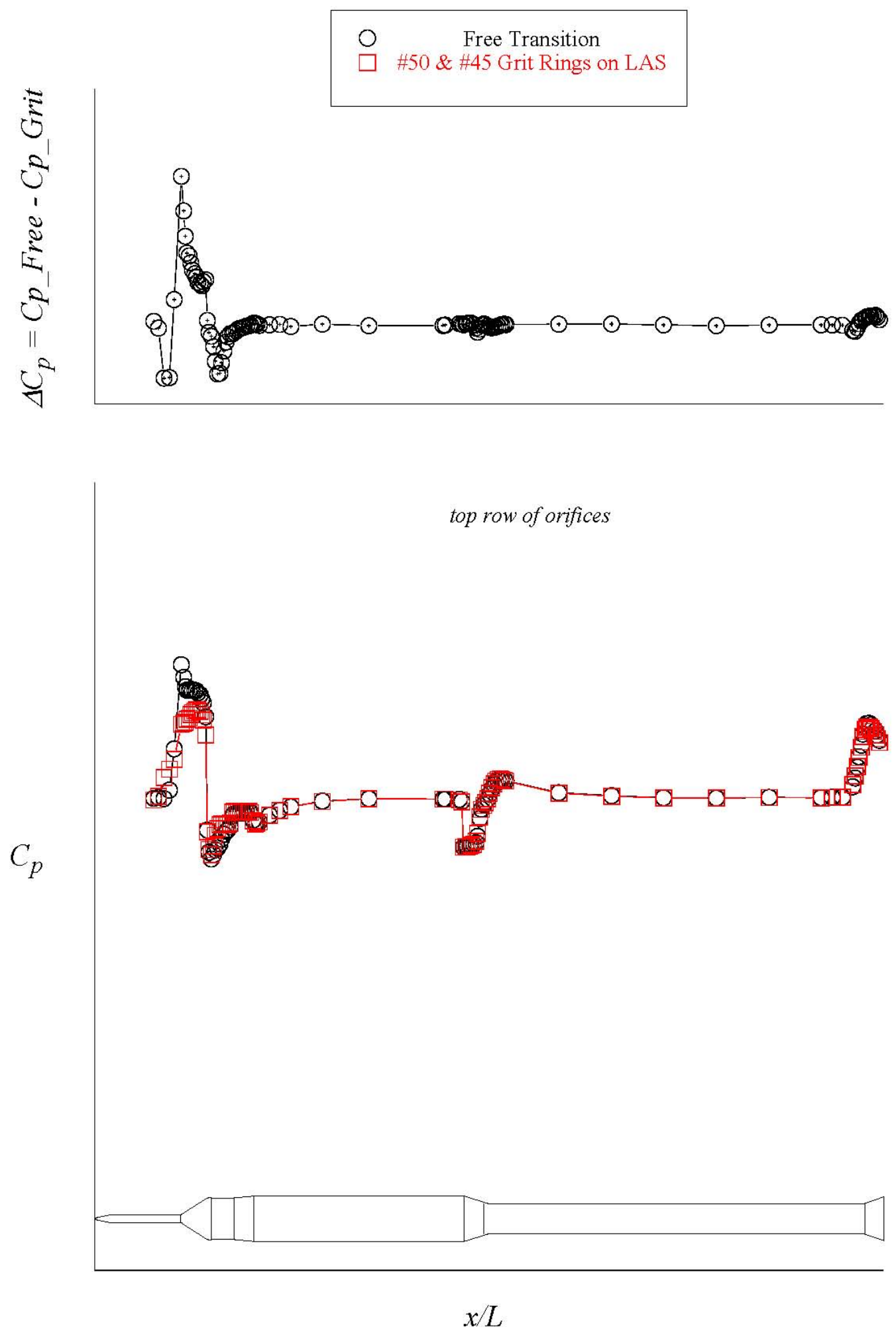

(b) \#50 and \#45 grit rings on LAS (default or baseline grit pattern in UPWT T. S. 1).

Fig. 21. Transition grit effect on the 0.01-scale Ares I ADAC-1 model pressure distributions at a selected Mach number and angle of attack in UPWT T. S. 1. 

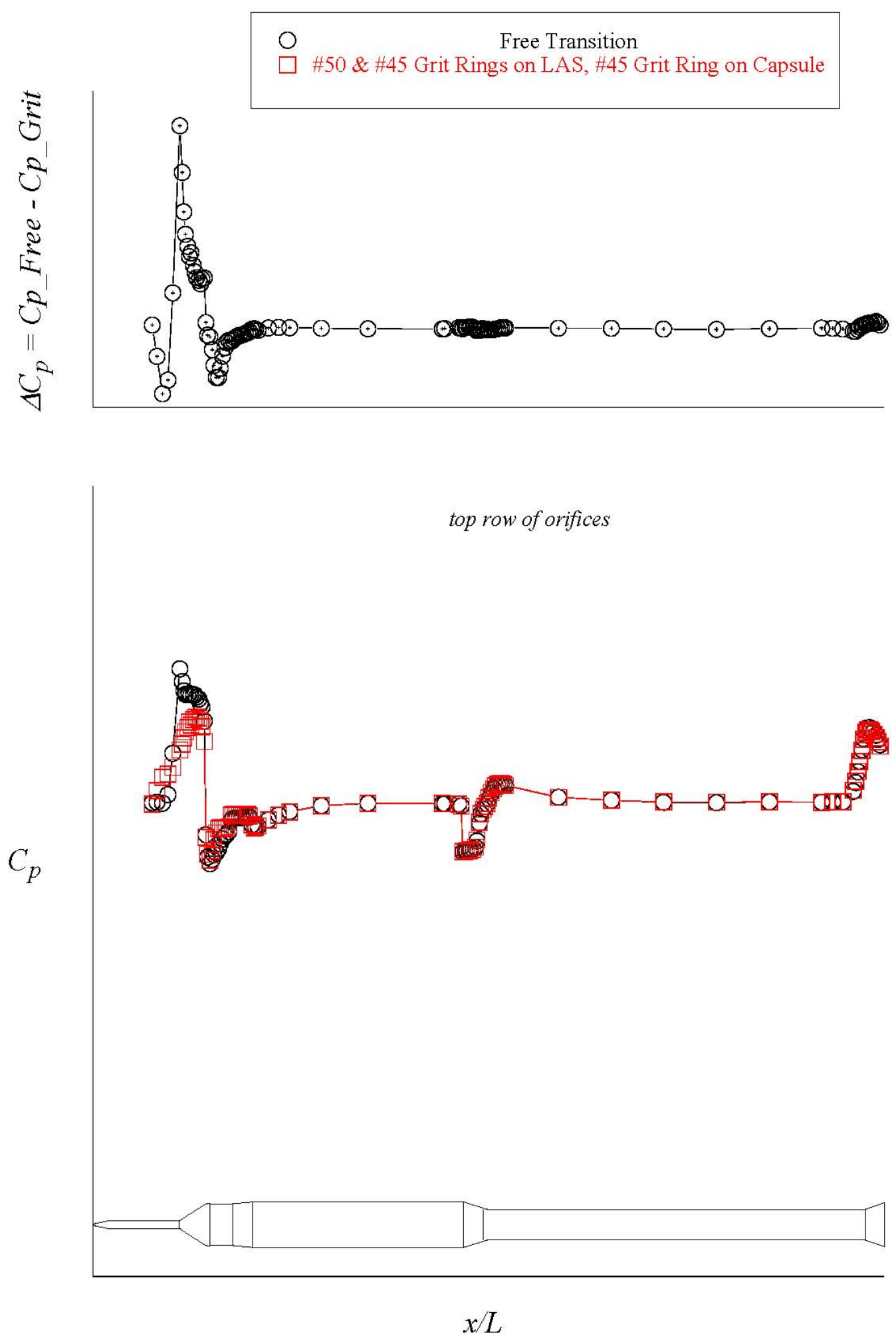

(c) \#50 and \#45 grit rings on LAS and \#45 grit ring on capsule.

Fig. 21. Transition grit effect on the 0.01-scale Ares I ADAC-1 model pressure distributions at a selected Mach number and angle of attack in UPWT T. S. 1. 

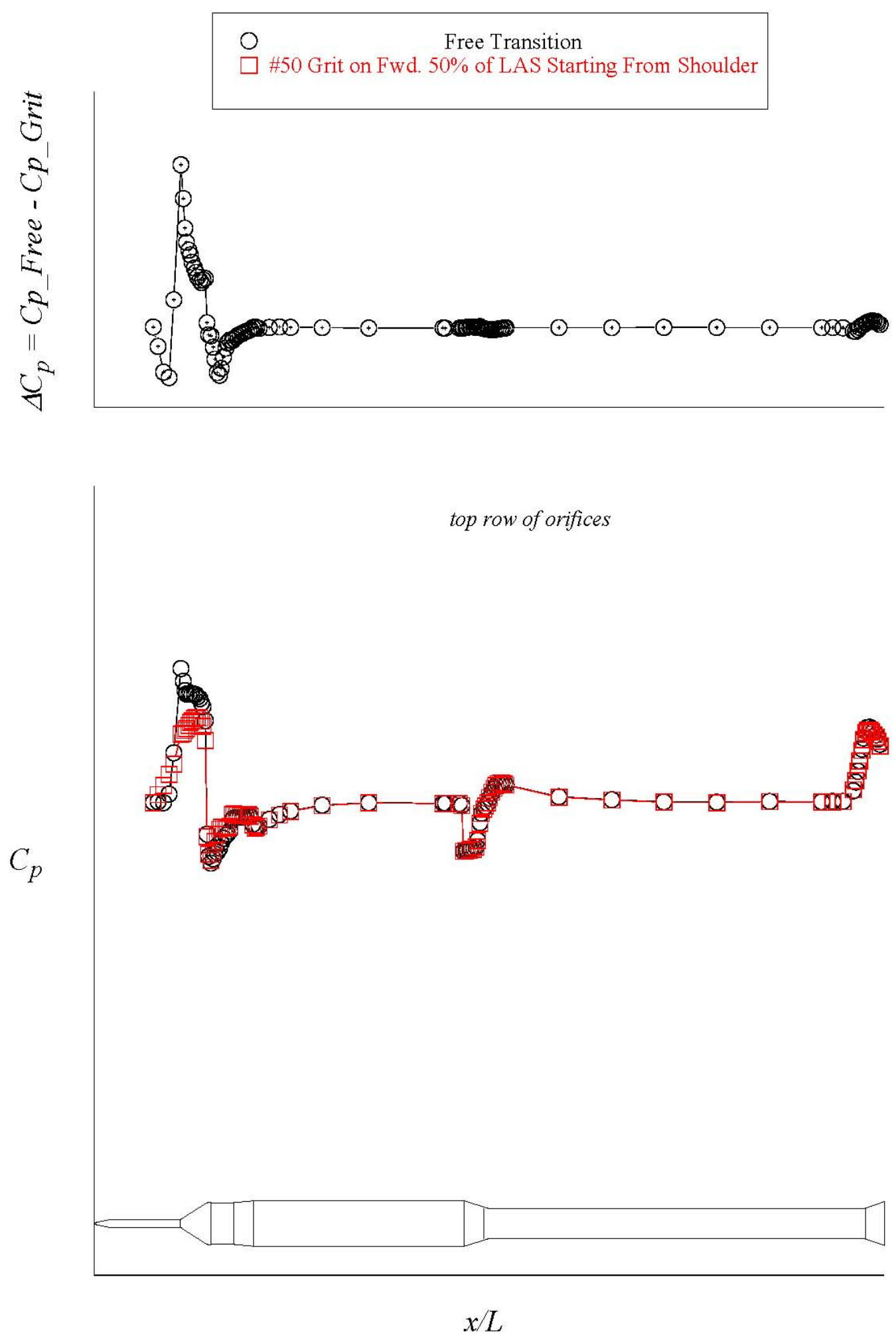

(d) \#50 grit on forward $50 \%$ of LAS starting from shoulder.

Fig. 21. Transition grit effect on the 0.01-scale Ares I ADAC-1 model pressure distributions at a selected Mach number and angle of attack in UPWT T. S. 1. 

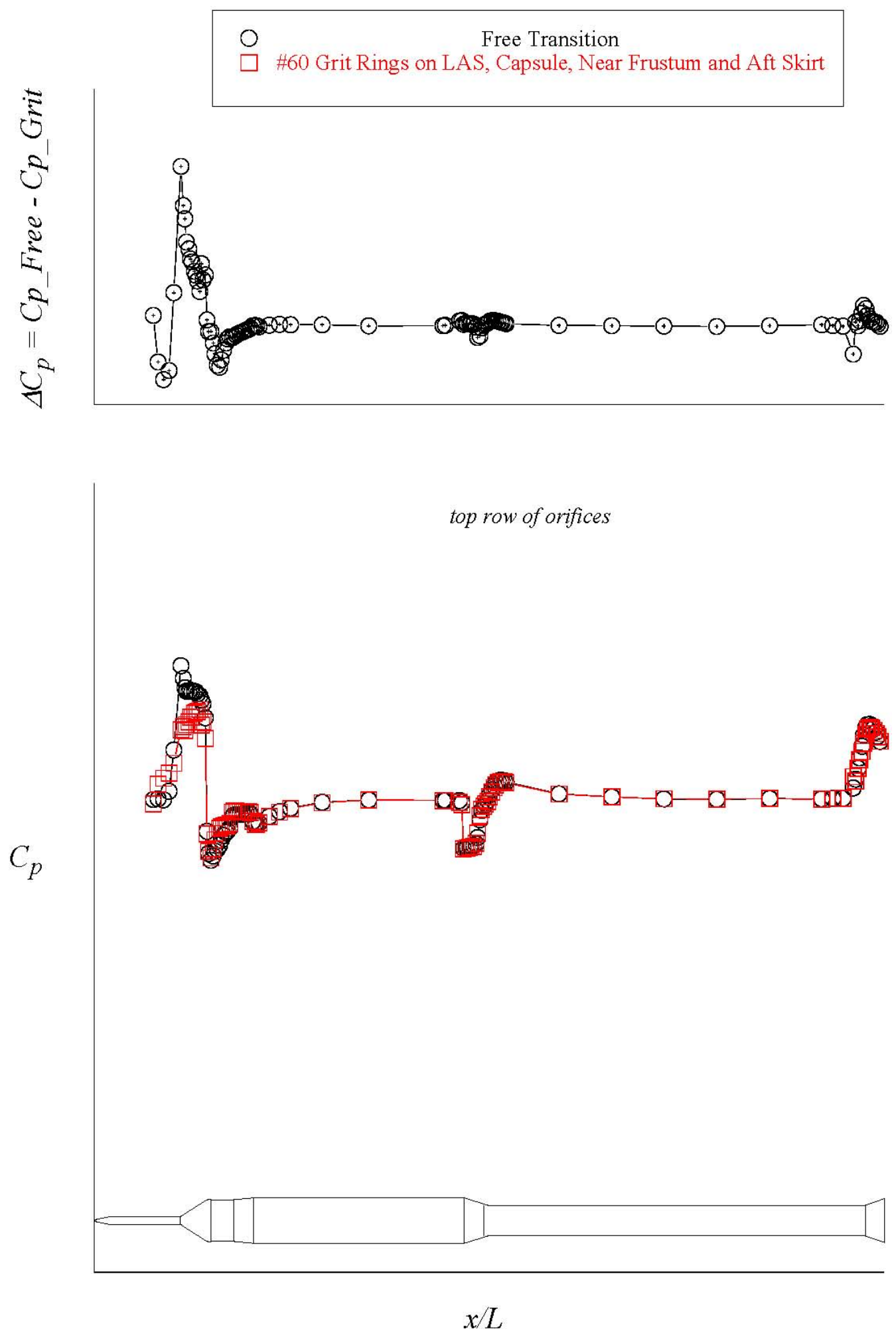

(e) \#60 grit rings on LAS, capsule, near frustum and aft skirt.

Fig. 21. Transition grit effect on the 0.01-scale Ares I ADAC-1 model pressure distributions at a selected Mach number and angle of attack in UPWT T. S. 1. 


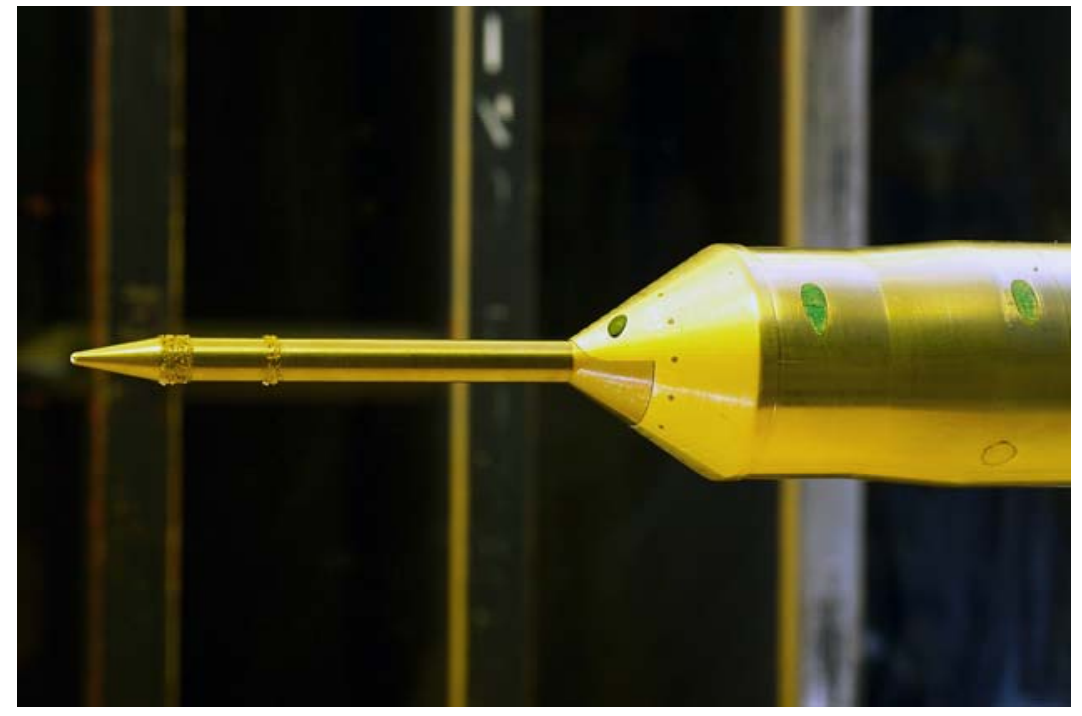

Fig. 22. Close-up photograph of baseline grit pattern on the 0.01-scale Ares I ADAC-1 pressure model installed in UPWT T. S. 1.
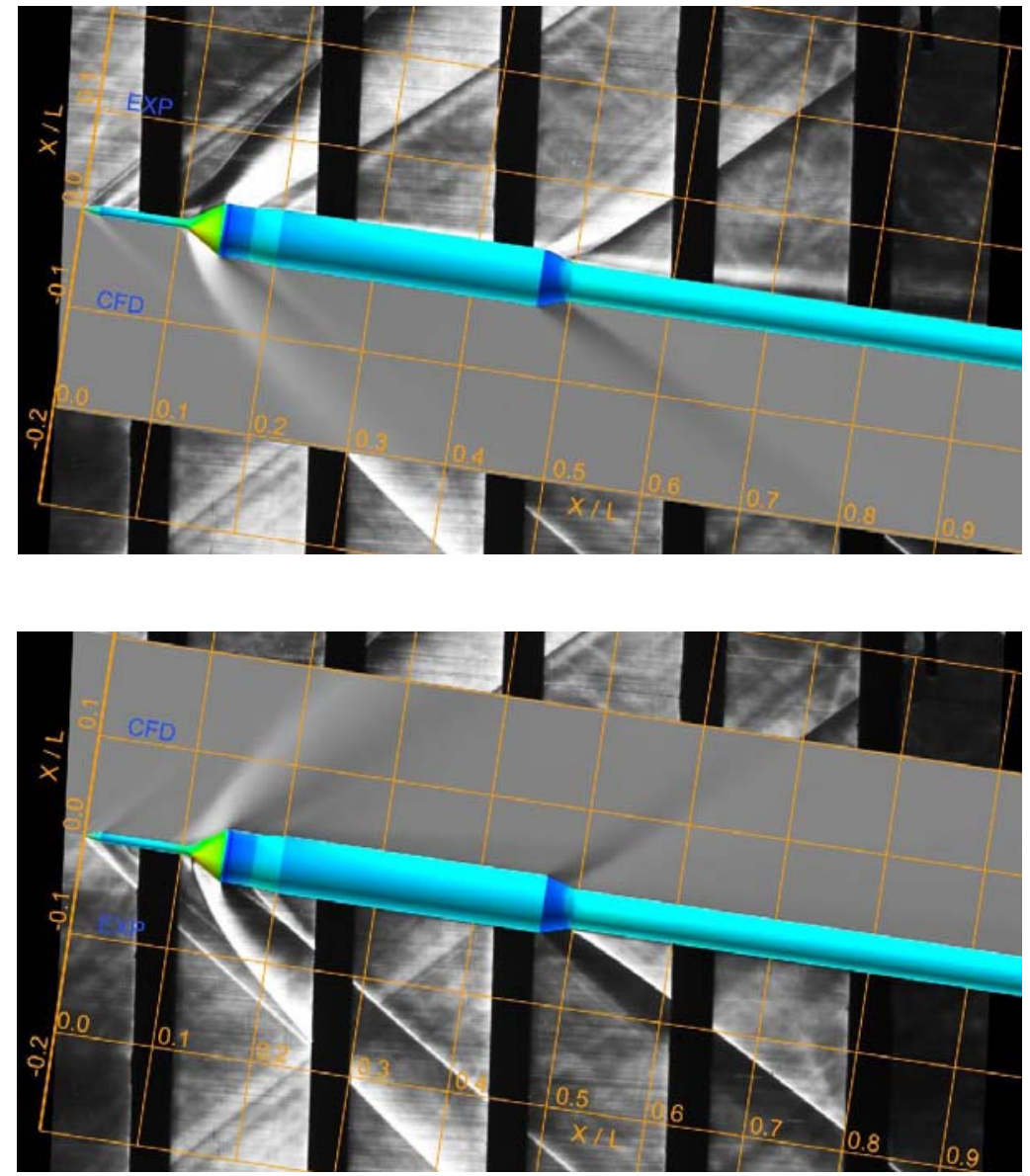

Fig. 23. Comparison of experimental schlieren images and CFD density contours at a selected supersonic Mach number on the 0.01-scale Ares I ADAC-1 pressure model in UPWT T. S. 1. 

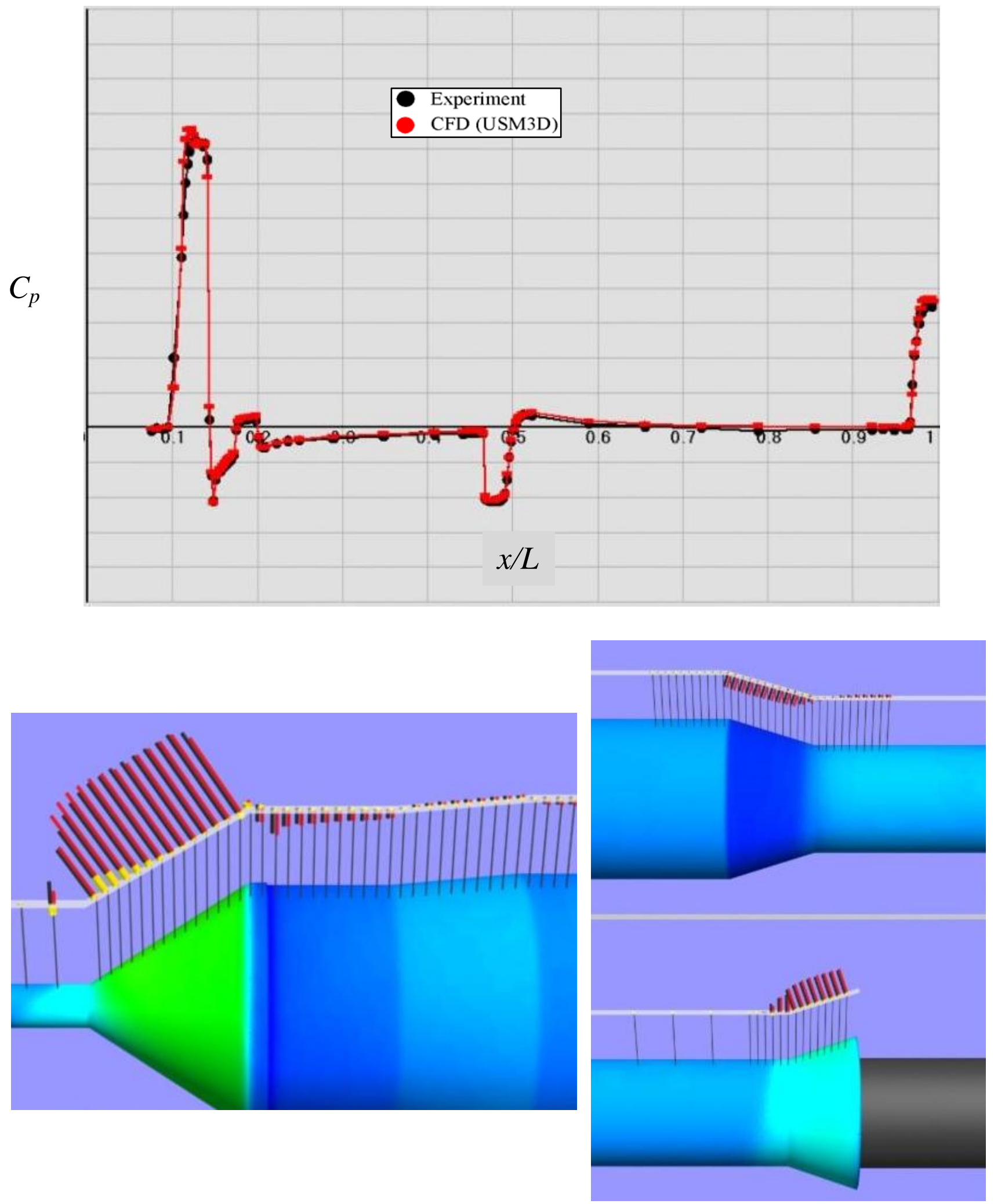

Fig. 24. Comparison of experimental and CFD pressure distributions on the 0.01-scale Ares I ADAC-1 model in UPWT T. S. 1 using real-time virtual diagnostics interface software. 

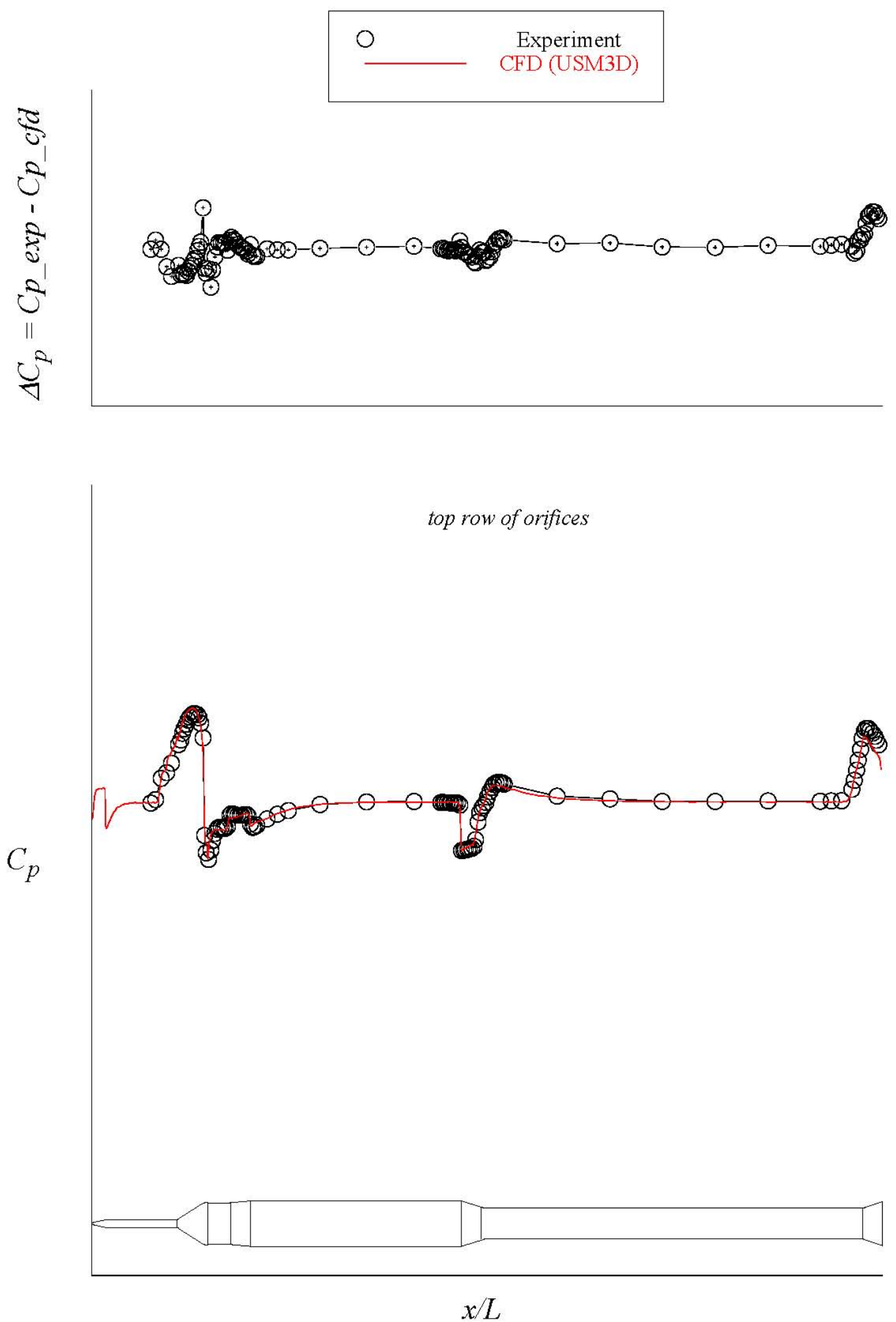

Fig. 25. 2-D plot comparison of experimental and CFD pressure distributions on the 0.01-scale Ares I ADAC-1 pressure model in UPWT T. S. 1. 


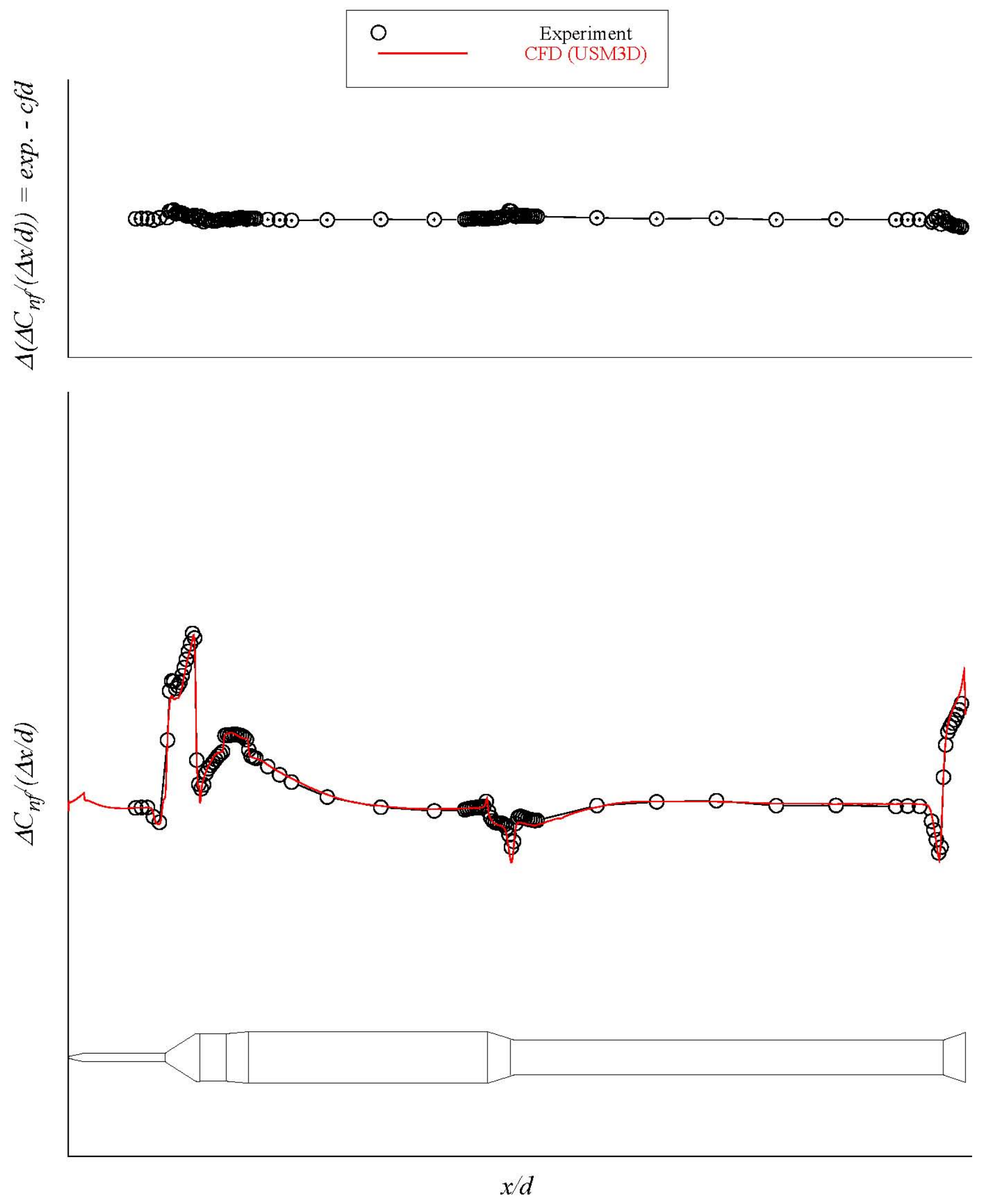

Fig. 26. Comparison of experimental and CFD longitudinal line-load distributions on the 0.01-scale Ares I ADAC-1 pressure model in UPWT T. S. 1. 

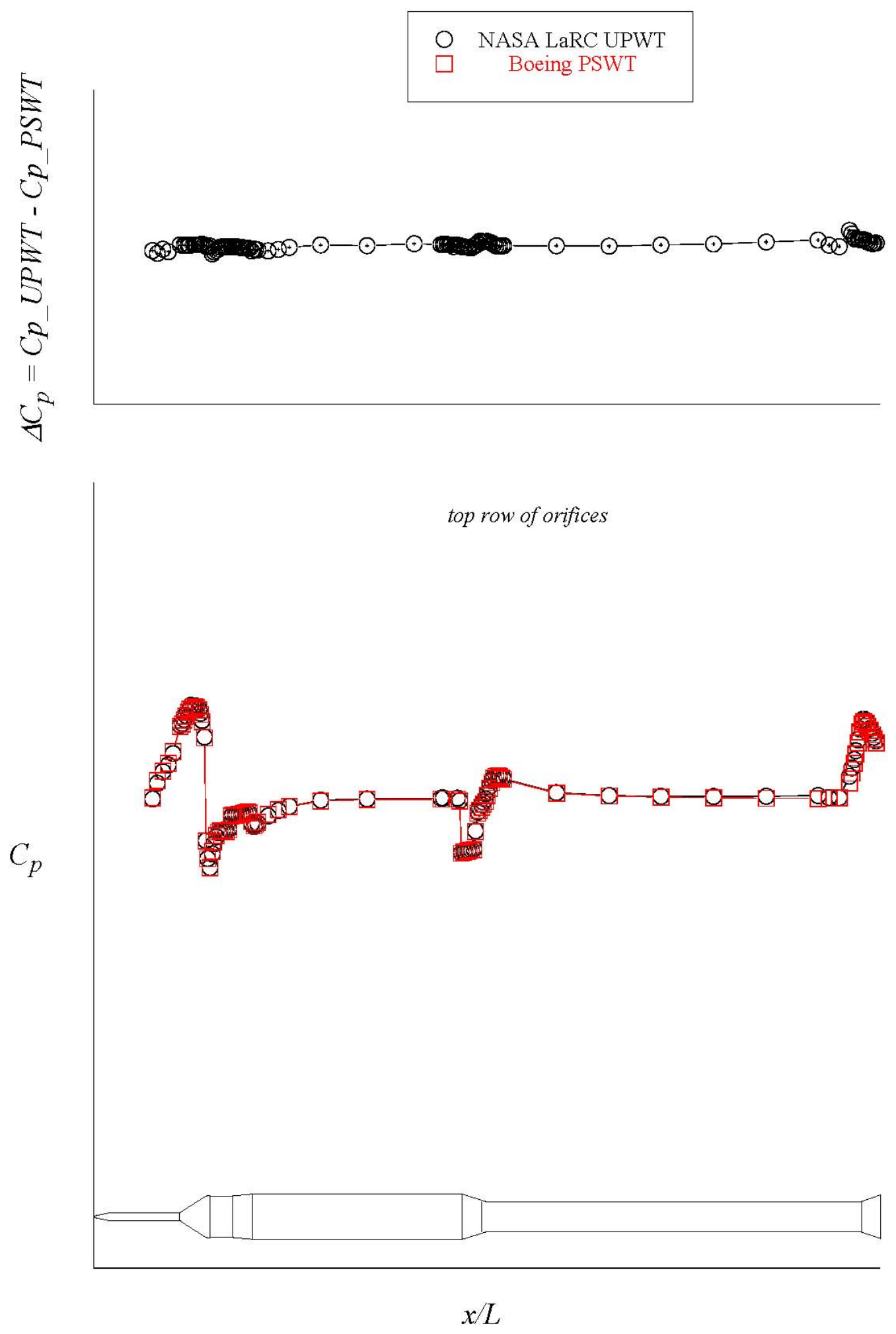

Fig. 27. Tunnel-to-tunnel comparisons of the pressure distributions on the 0.01-scale Ares I ADAC-1 model at a selected angle of attack and supersonic Mach number in UPWT T. S. 1 and Boeing PSWT. 


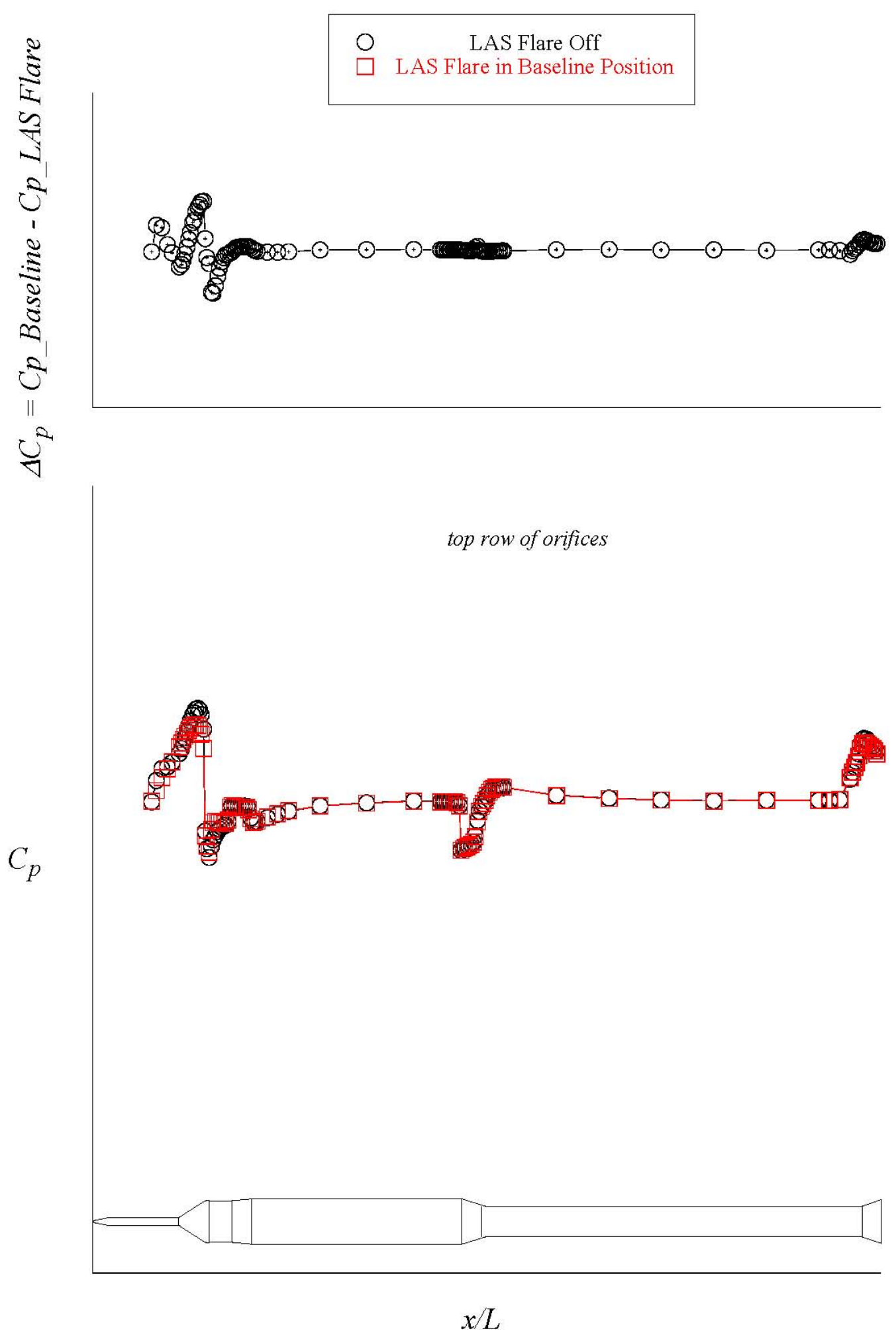

(a) roll angle $=0$ degrees.

Fig. 28. Effect of LAS flare in baseline position on the 0.01-scale Ares I ADAC-1 model pressure distributions at a selected angle of attack and lower supersonic Mach number in UPWT T. S. 1. 


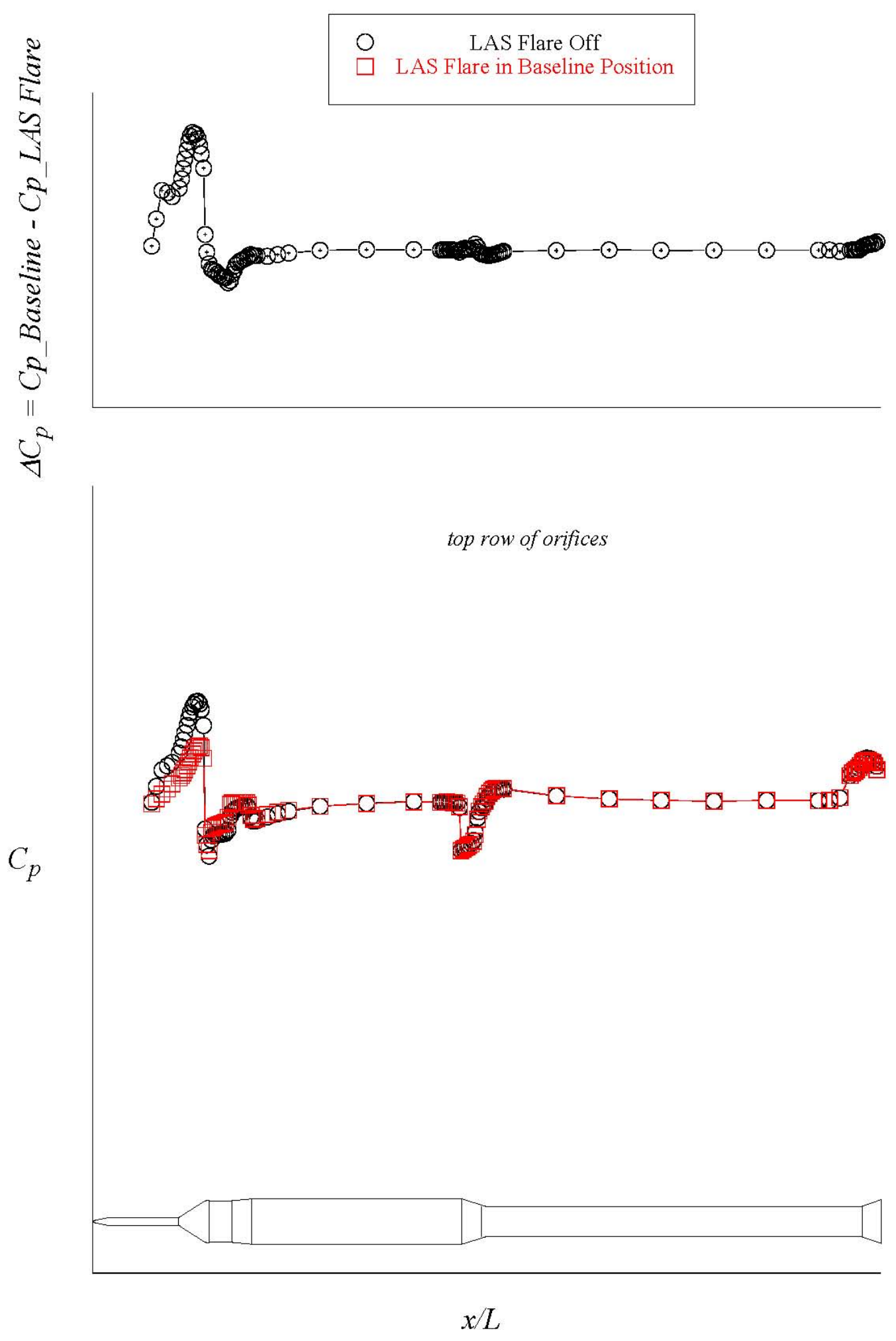

(b) roll angle $>0$ degrees.

Fig. 28. Effect of LAS flare in baseline position on the 0.01-scale Ares I ADAC-1 model pressure distributions at a selected angle of attack and lower supersonic Mach number in UPWT T. S. 1. 


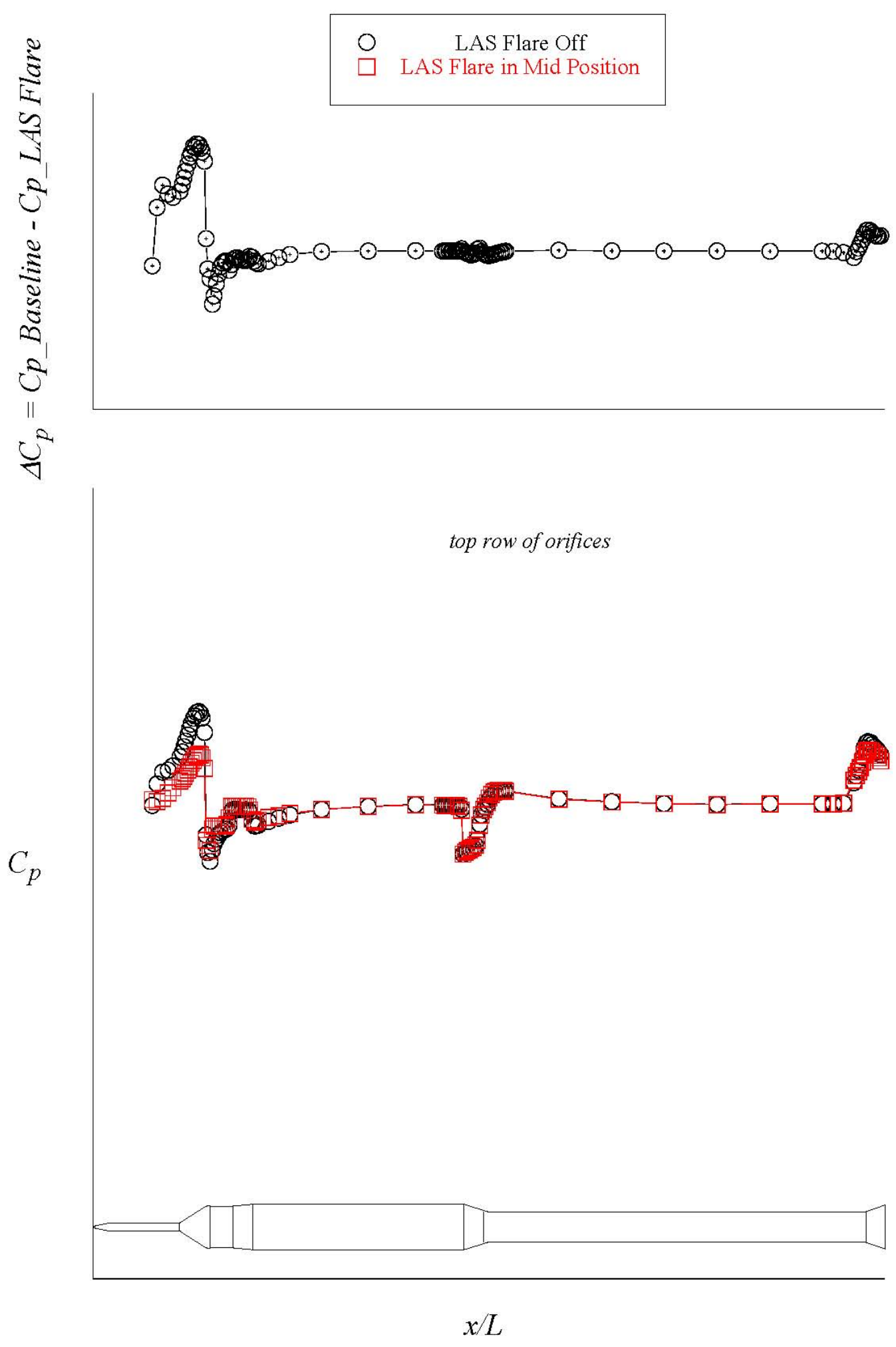

(a) roll angle $=0$ degrees.

Fig. 29. Effect of LAS flare in mid position on the 0.01-scale Ares I ADAC-1 model pressure distributions at a selected angle of attack and lower supersonic Mach number in UPWT T. S. 1. 


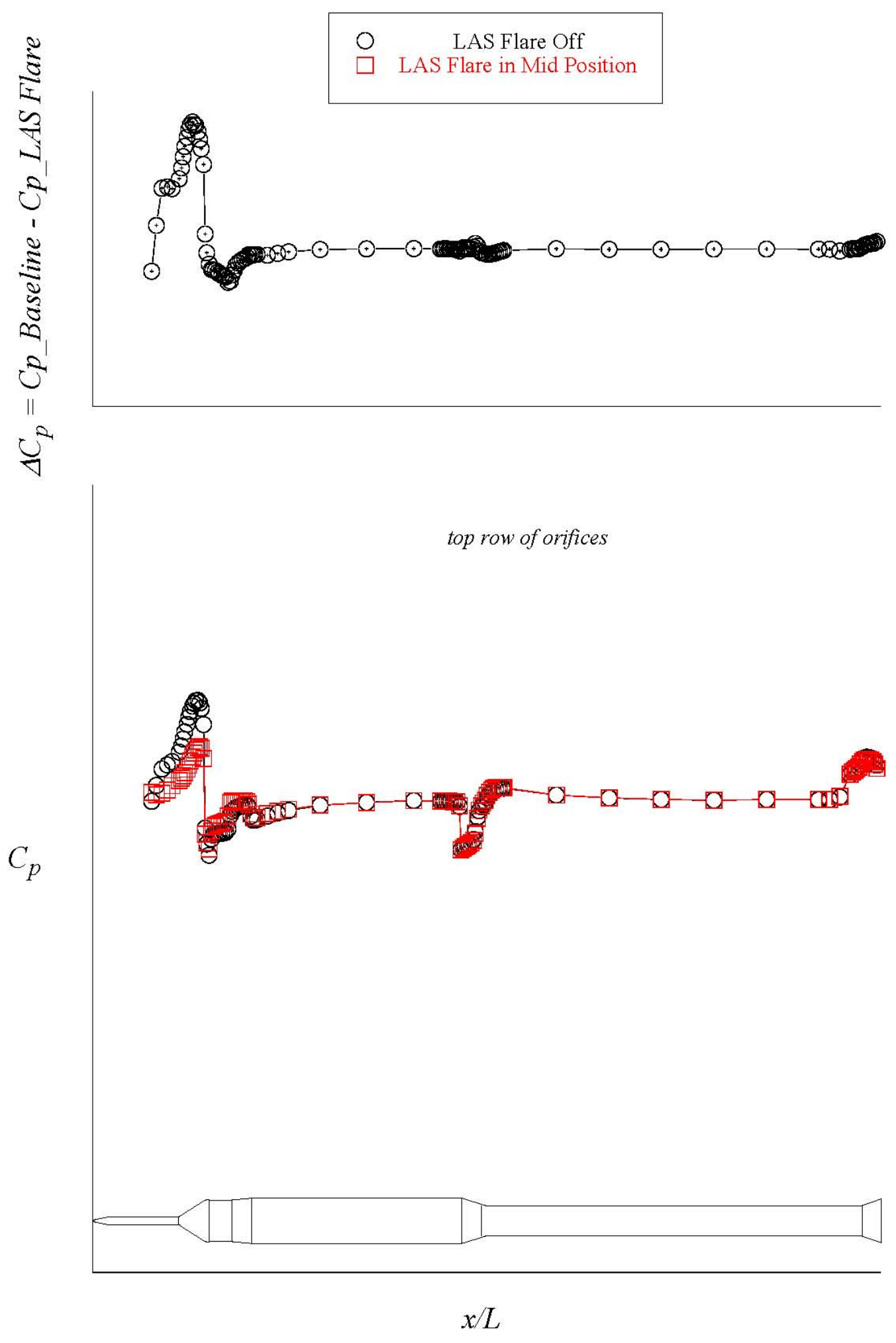

(b) roll angle $>0$ degrees.

Fig. 29. Effect of LAS flare in mid position on the 0.01-scale Ares I ADAC-1 model pressure distributions at a selected angle of attack and lower supersonic Mach number in UPWT T. S. 1. 


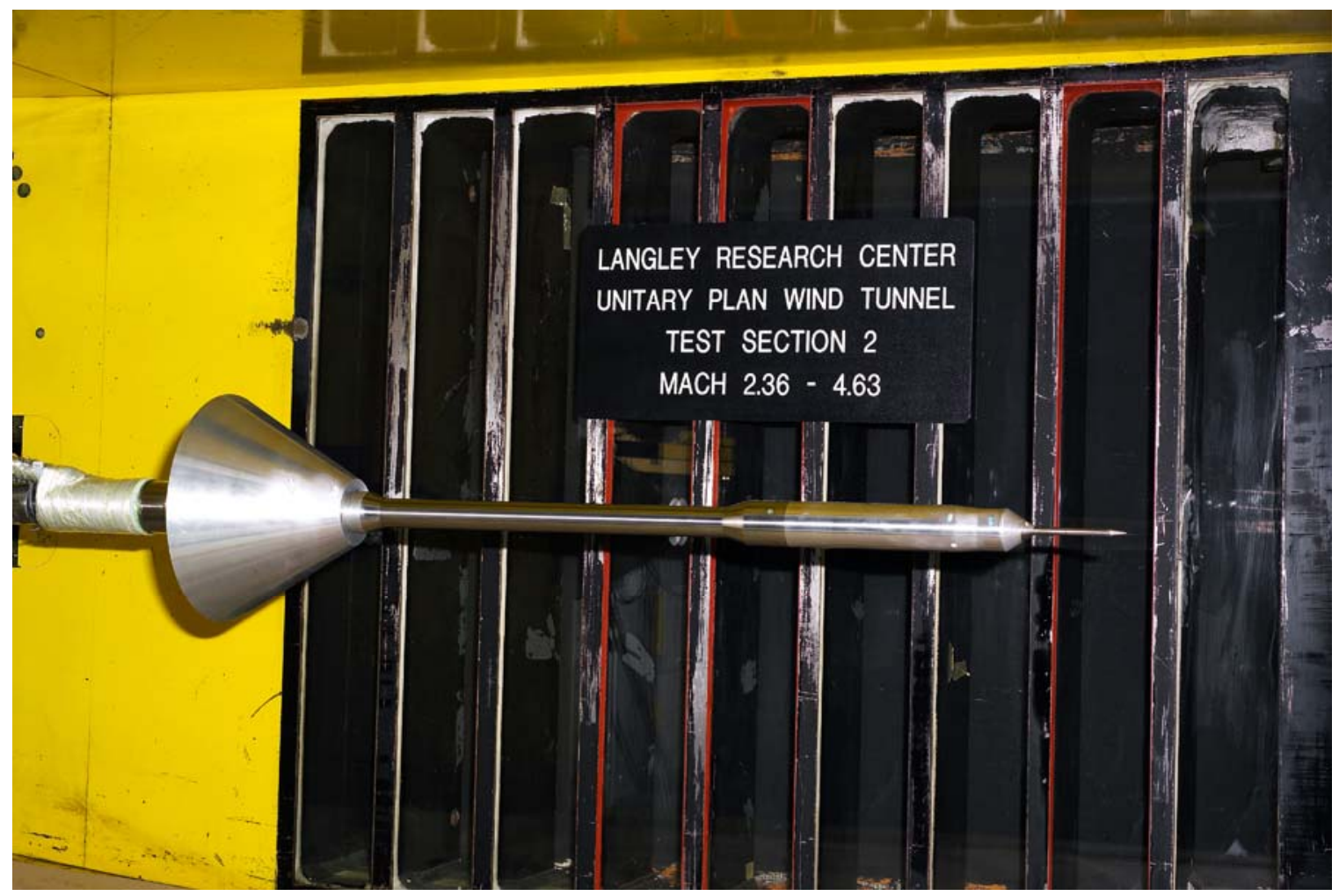

Fig. 30. Photograph of the 0.01-scale Ares I ADAC-1 pressure model with solid plume in UPWT T. S. 2.

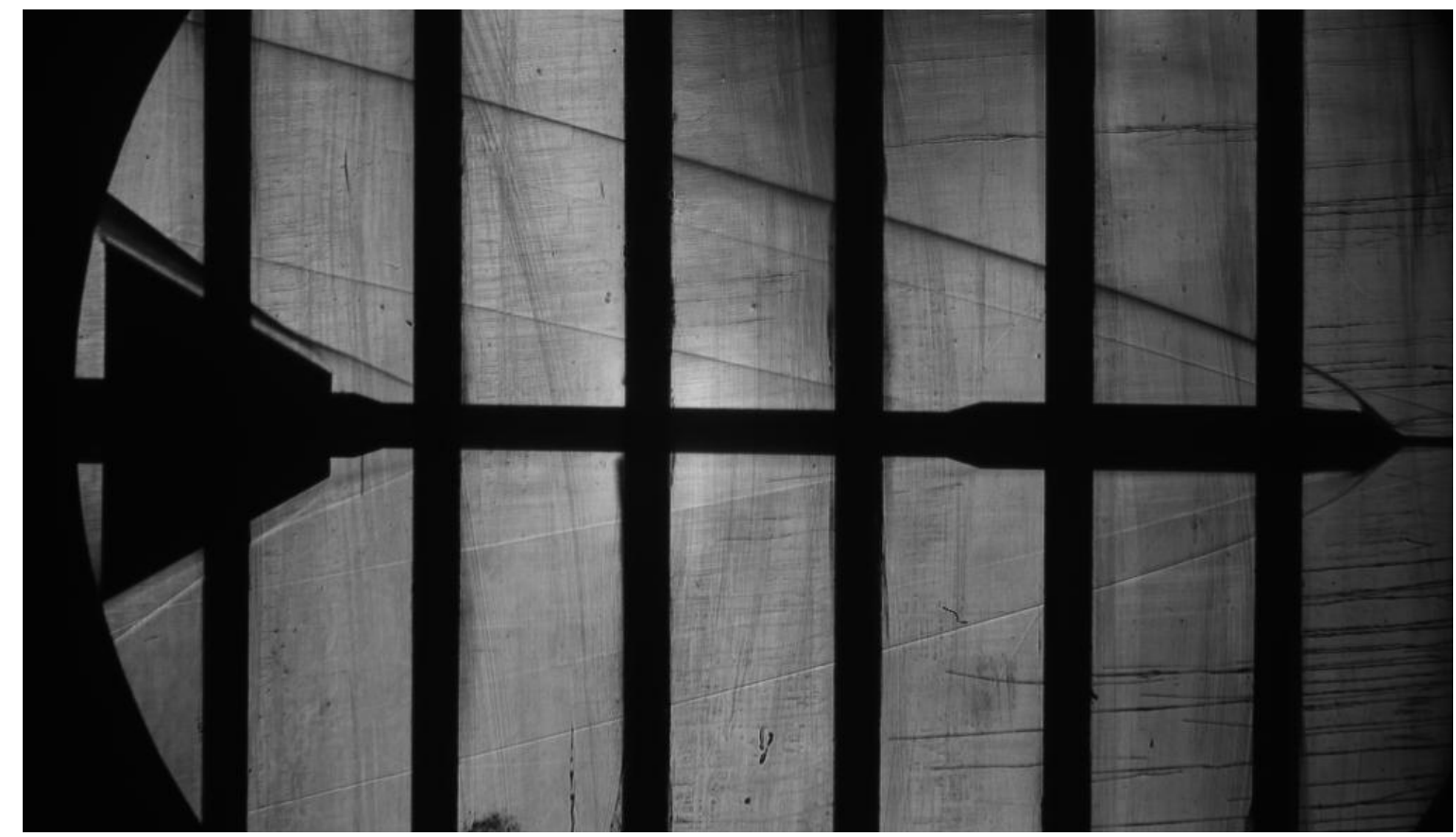

Fig. 31. Schlieren flow visualization image of the 0.01-scale Ares I ADAC-1 pressure model at a high supersonic Mach number in UPWT T. S. 2. 


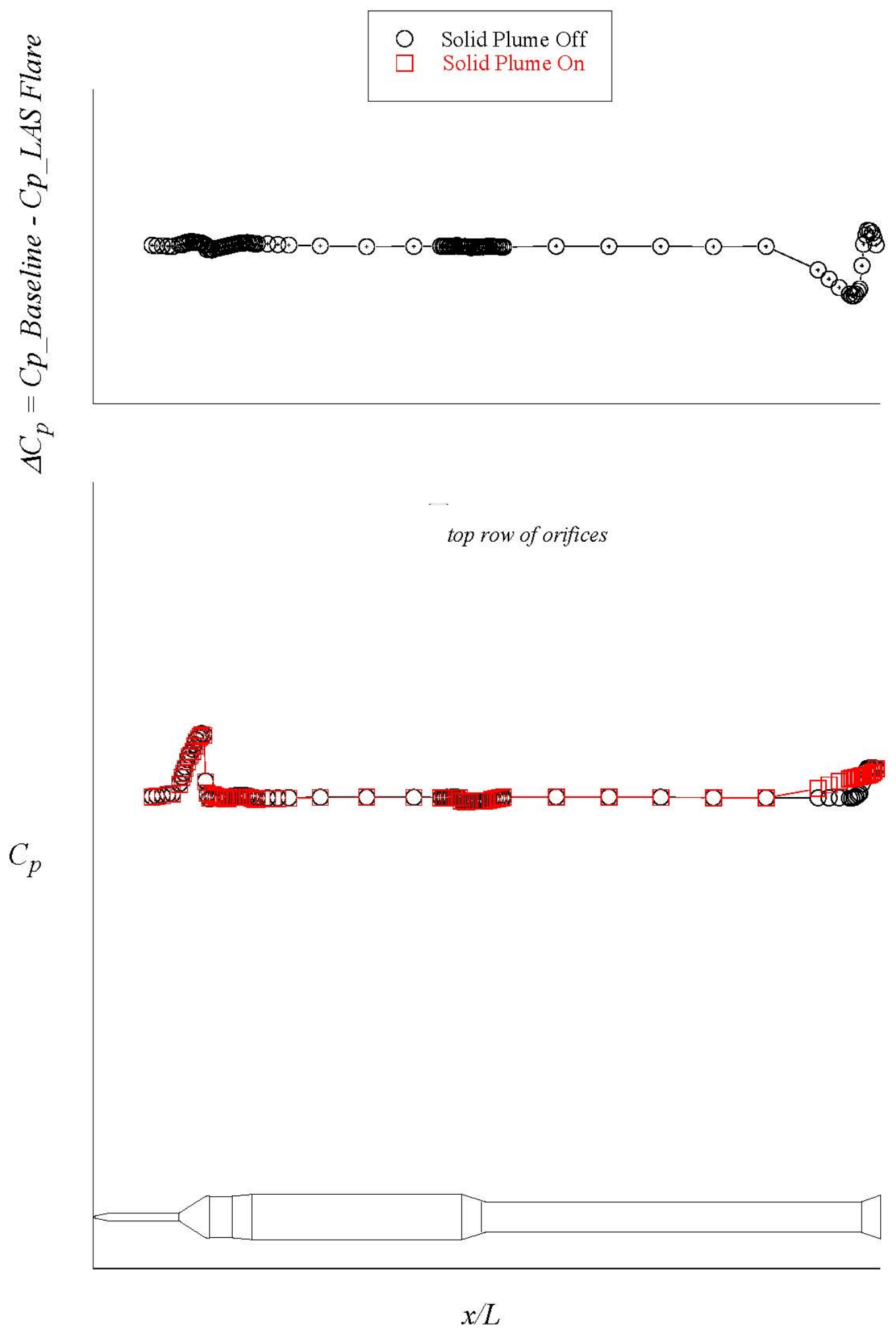

Fig. 32. Solid plume effect on the 0.01-scale Ares I ADAC-1 model pressure distributions at a selected angle of attack and a high supersonic Mach number in UPWT T. S. 2. 


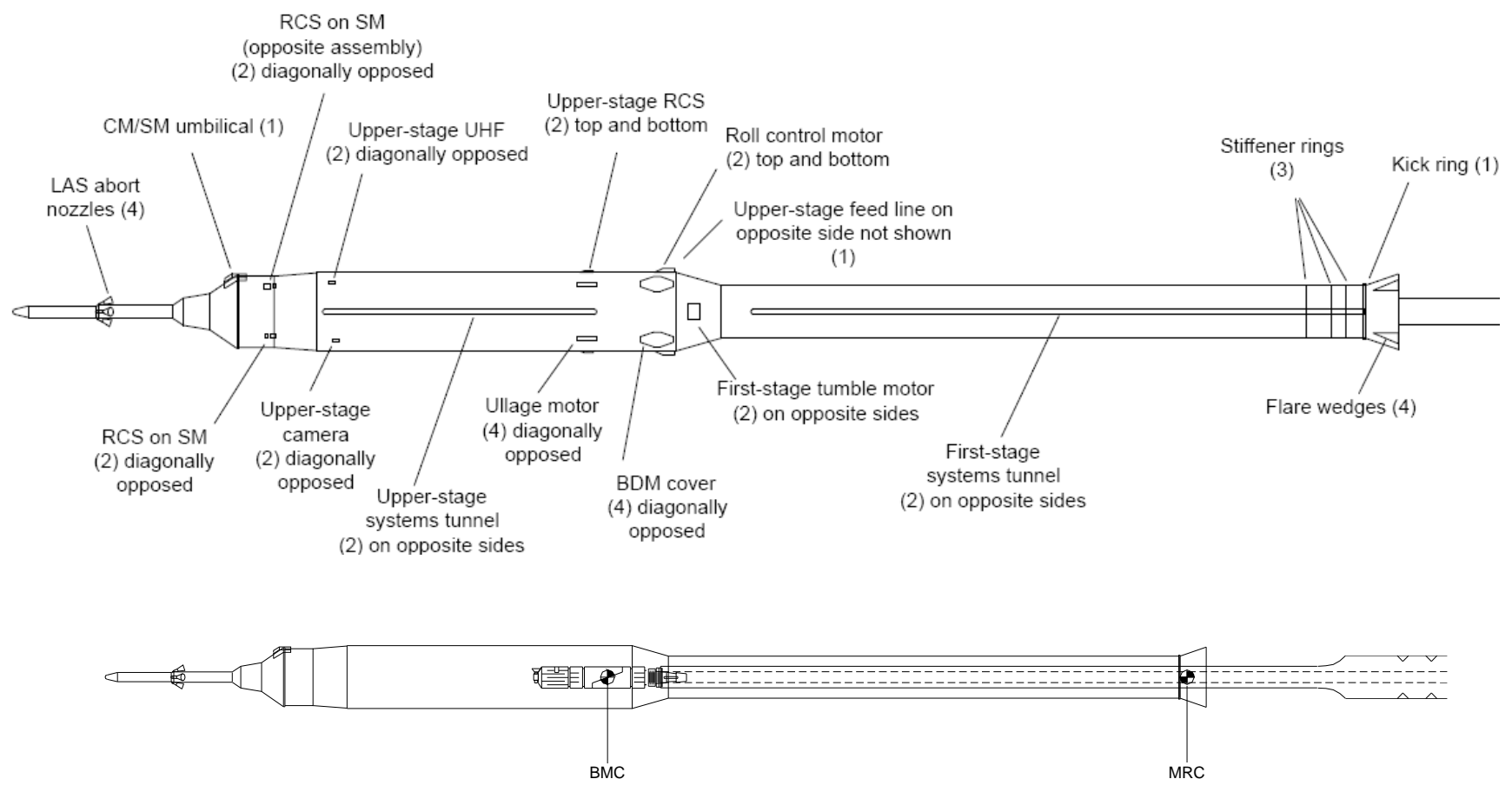

Fig. 33. Sketch of the Ares I ADAC-2A with protuberances (lower sketch shows location of MRC for aerodynamic moment coefficient computation).

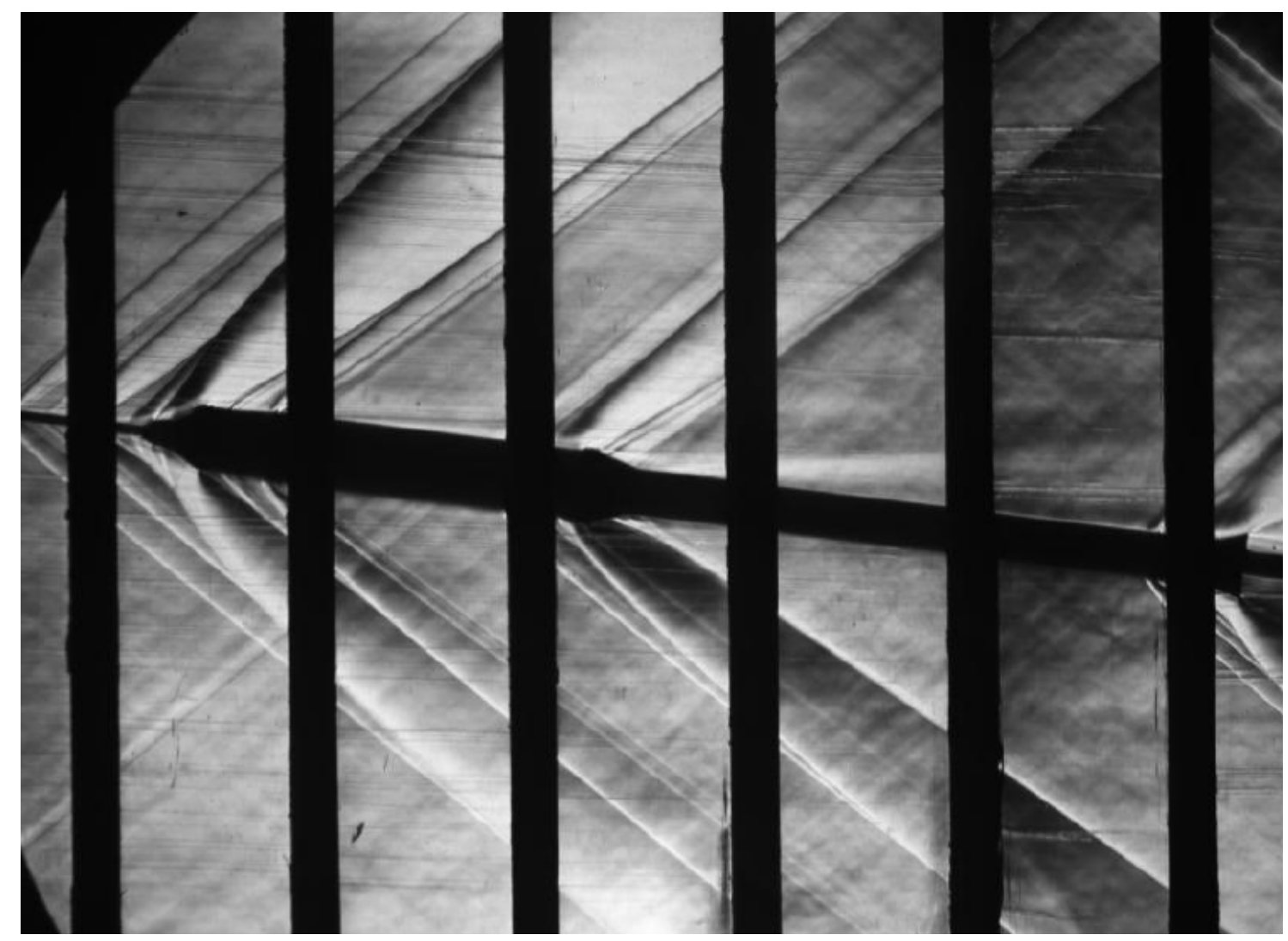

Fig. 34. Schlieren flow visualization image of the baseline 0.01-scale Ares I ADAC-2A model at a selected supersonic Mach number in UPWT T. S. 1. 


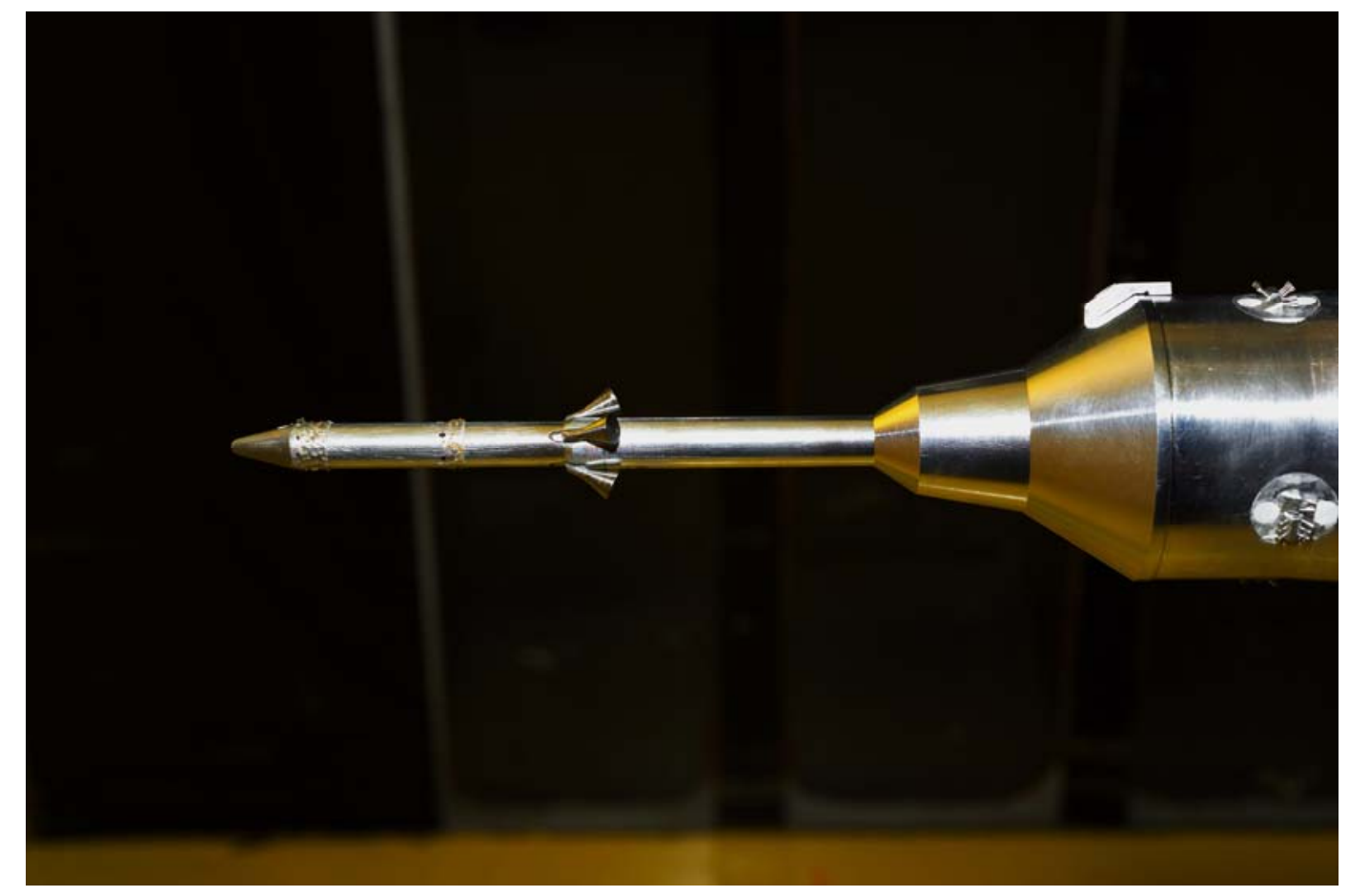

(a) Baseline party-hat shape.

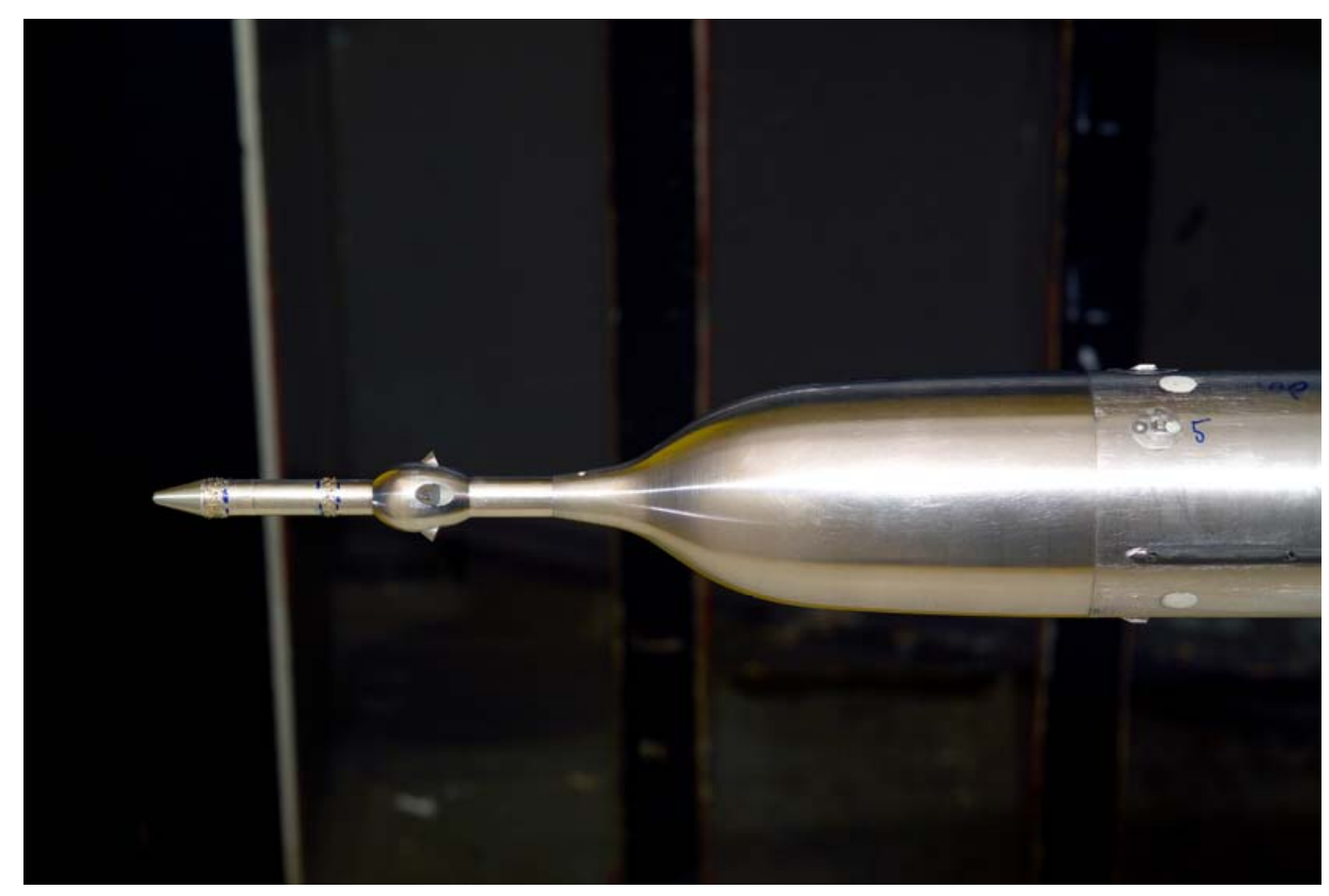

(b) Modified ogive shape with bulbous fairing over LAS nozzles (ALAS-11).

Fig. 35. Close-up photographs of the baseline party-hat and modified ogive BPC designs on the 0.01-scale Ares I ADAC-2A model in UPWT T. S. 1. 


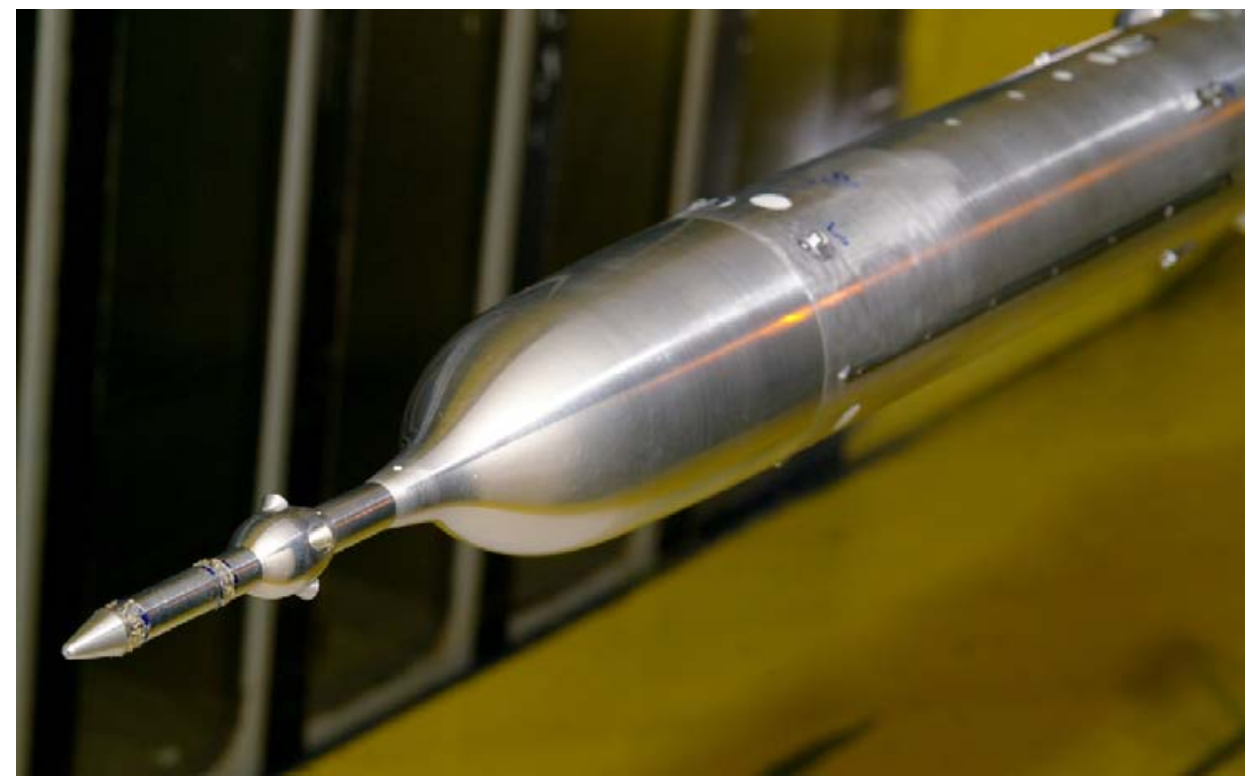

(c) Alternate view of the modified ogive design (ALAS-11).

Fig. 35. Close-up photographs of the baseline party-hat and modified ogive BPC designs on the 0.01-scale Ares I ADAC-2A model in UPWT T. S. 1.

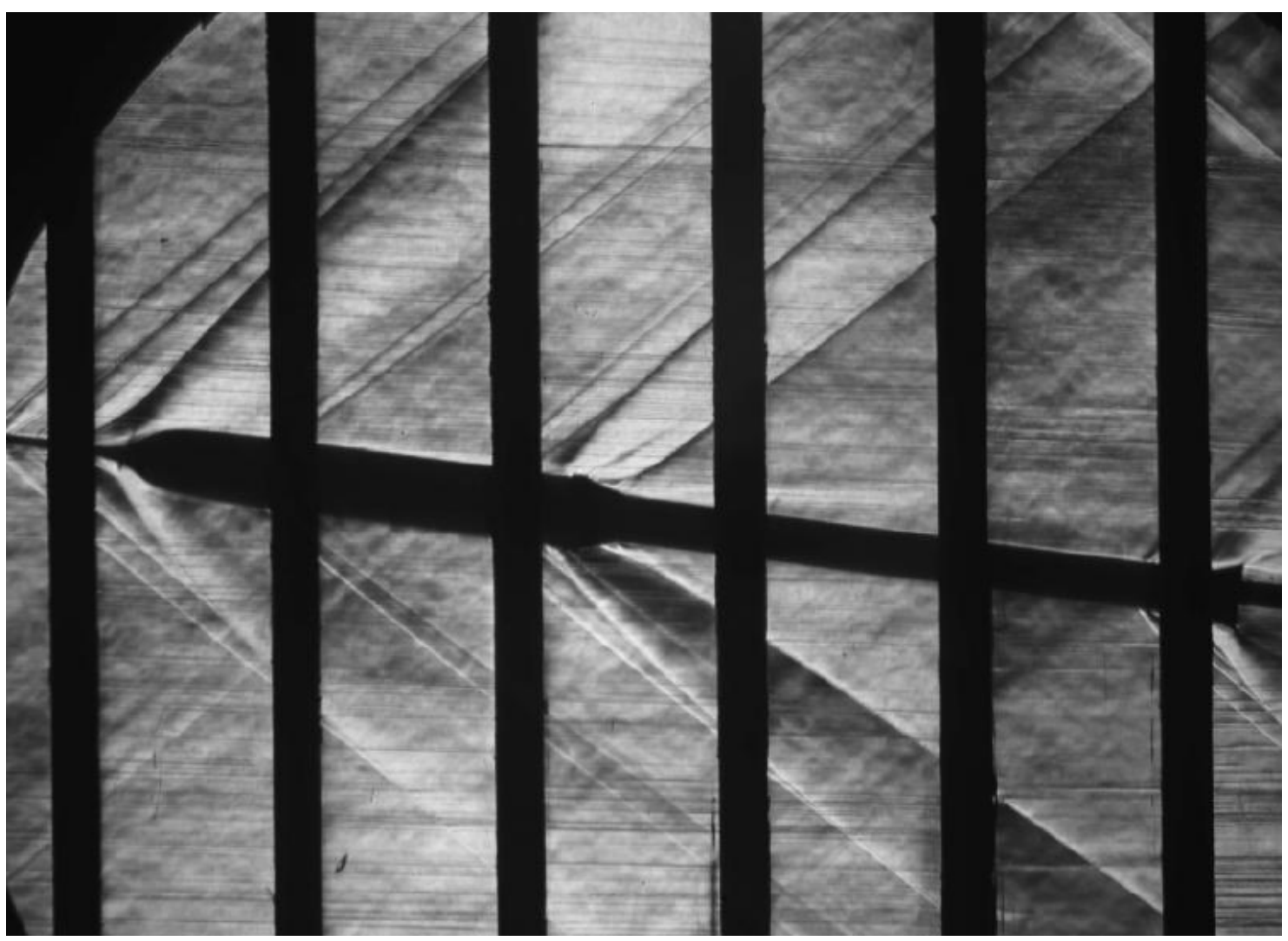

Fig. 36. Schlieren flow visualization image of the 0.01-scale Ares I ADAC-2A model with ogive ALAS-11 at a selected supersonic Mach number in UPWT T. S. 1. 


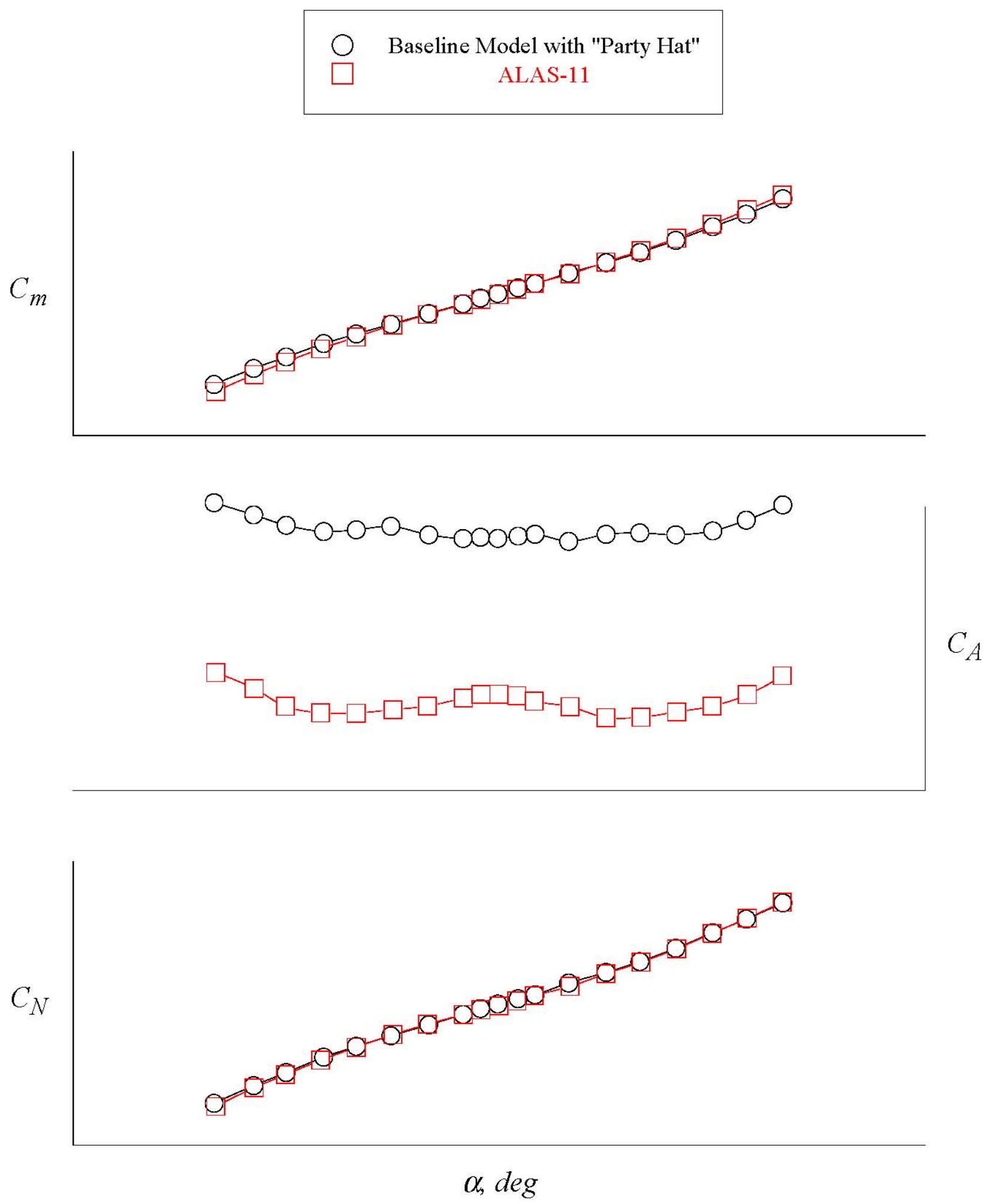

Fig. 37. Effect of modified BPC over CEV (ALAS-11) on the longitudinal aerodynamic characteristics of the 0.01-scale Ares I ADAC-2A model at a selected Mach number in UPWT T. S. 1. 

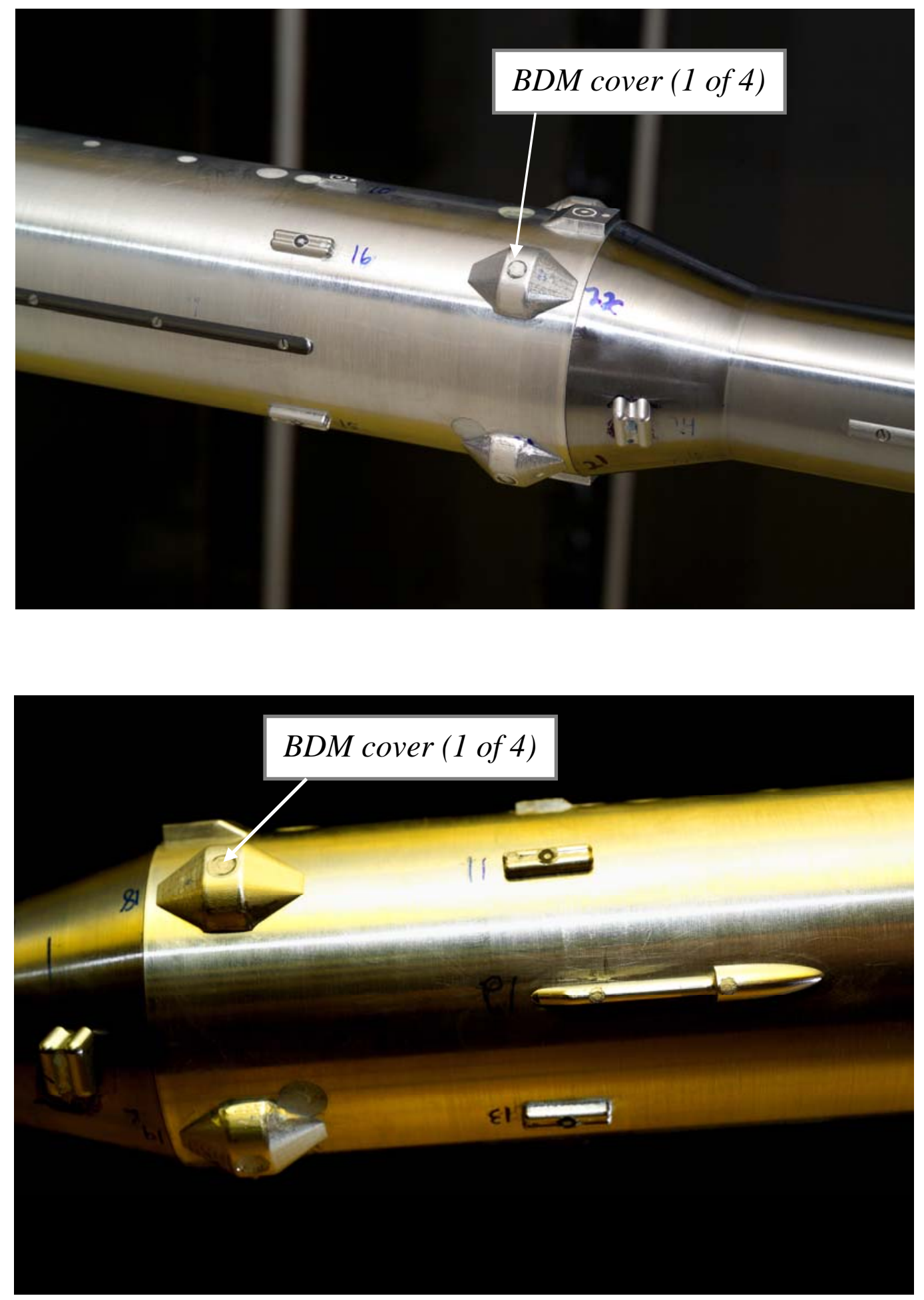

Fig. 38. Close-up photographs of the booster deceleration motor (BDM) covers on the 0.01-scale Ares I ADAC-2A model installed in UPWT T. S. 1. 

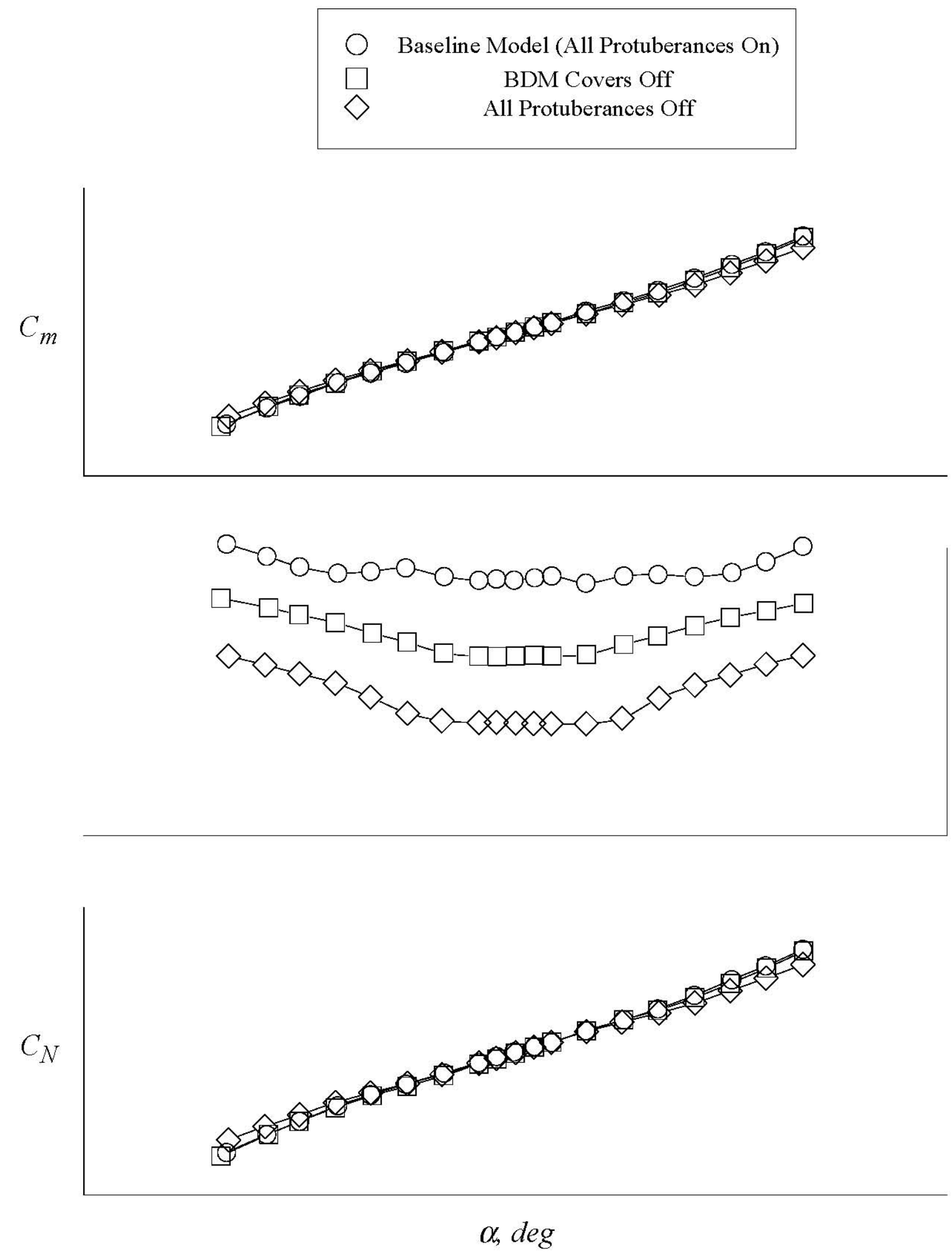

Fig. 39. Effect of protuberances on the longitudinal aerodynamic characteristics of the 0.01-scale Ares I ADAC-2A model at a selected Mach number in UPWT T. S. 1. 

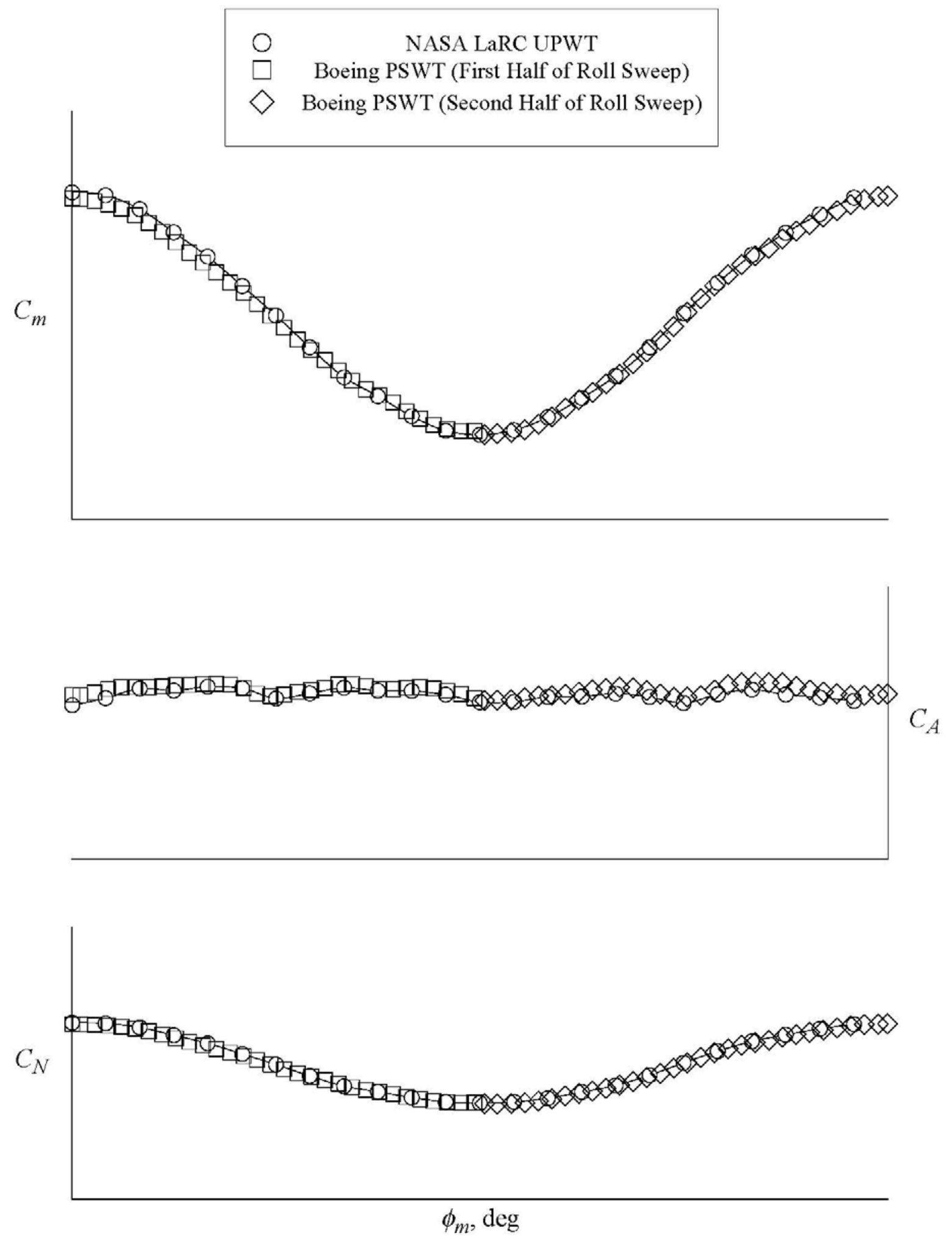

(a) Longitudinal aerodynamic characteristics.

Fig. 40. Tunnel-to-tunnel comparisons of the six-component force and moment coefficients on the baseline 0.01-scale Ares I ADAC-2A model in UPWT T. S. 1 and Boeing PSWT; roll angle sweeps at constant angle of attack. (All coefficients are in the body-axis coordinate system.) 

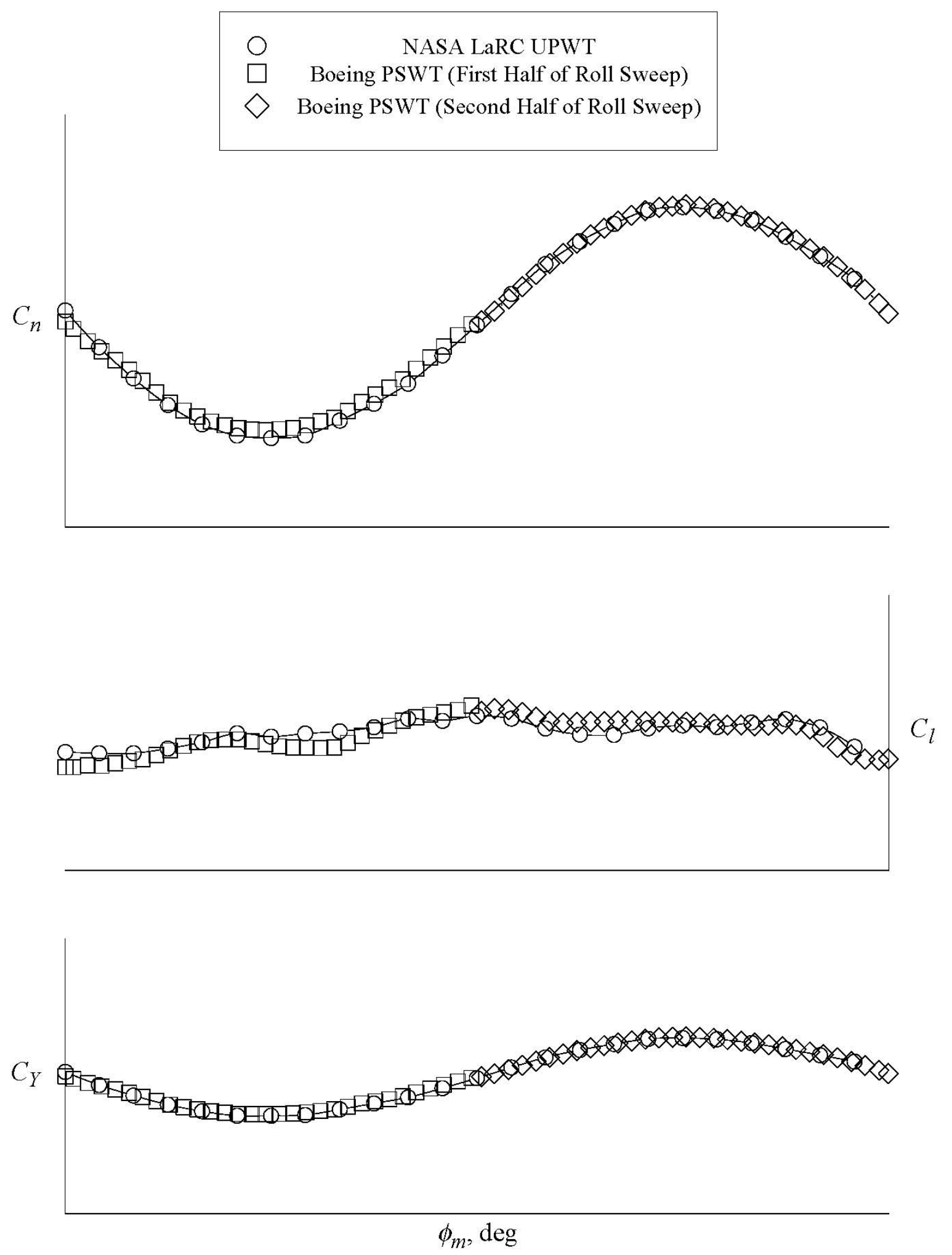

(b) Lateral-directional aerodynamic characteristics.

Fig. 40. Tunnel-to-tunnel comparisons of the six-component force and moment coefficients on the baseline 0.01-scale Ares I ADAC-2A model in UPWT T. S. 1 and Boeing PSWT; roll angle sweeps at constant angle of attack. (All coefficients are in the body-axis coordinate system.) 


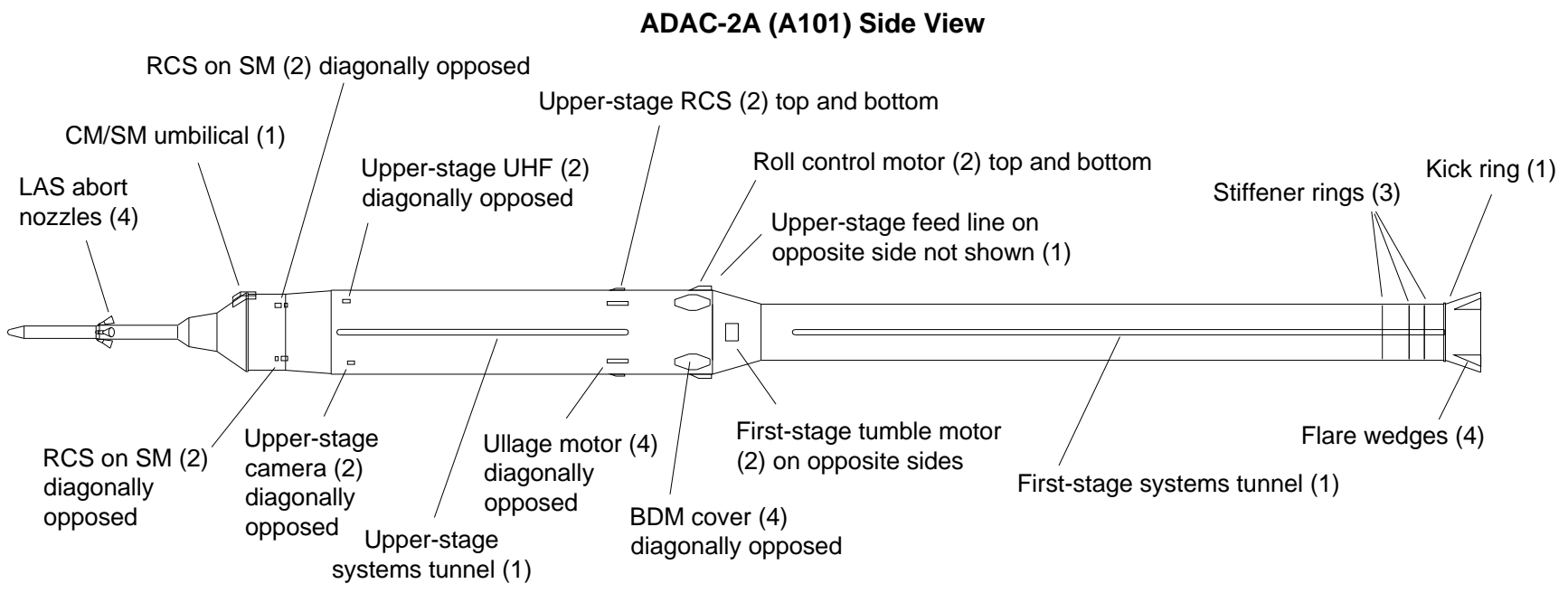

(a) Ares I ADAC-2A side view.

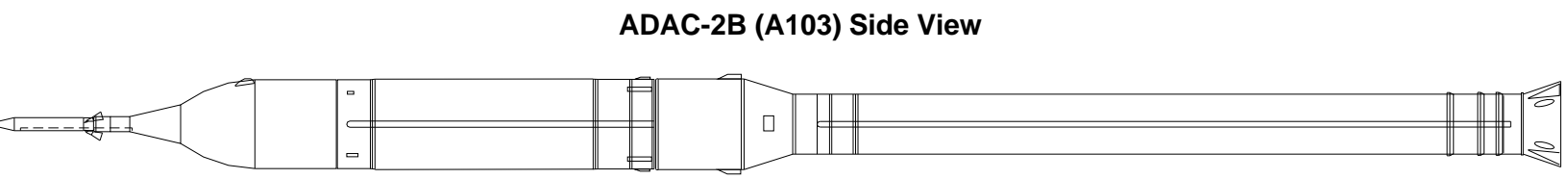

\section{ADAC-2B (A103) Top View}

upper stage LH2 feedline (1)

upper stage feedline (1)

upper stage camera (2)

LAS nozzles (4)

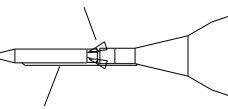

LAS tunnel (1)

capsule umbilical (1)

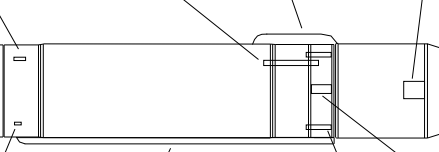

roll control motor (2)

1st stage tumble motor (2)

BDM motor (8)

upper stage tunnel (1)

1S

upper stage RCS

ullage motor (4)

(b) Ares I ADAC-2B side view and top view.

Figure 41. Schematic of Ares I ADAC-2B with protuberances. (Ares I ADAC-2A is shown for reference.) 


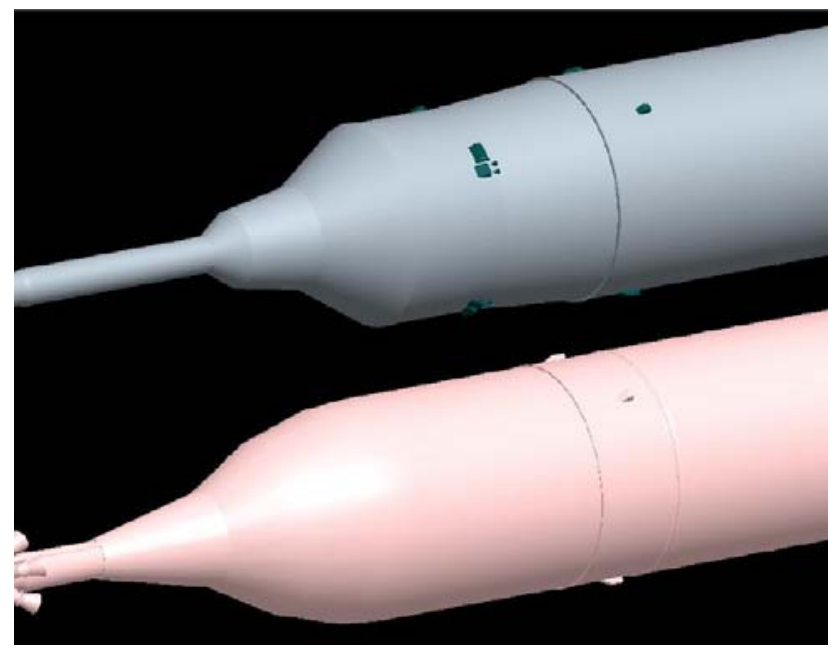

(a) CAD depiction of ADAC-2A (top) and ADAC-2B (bottom).
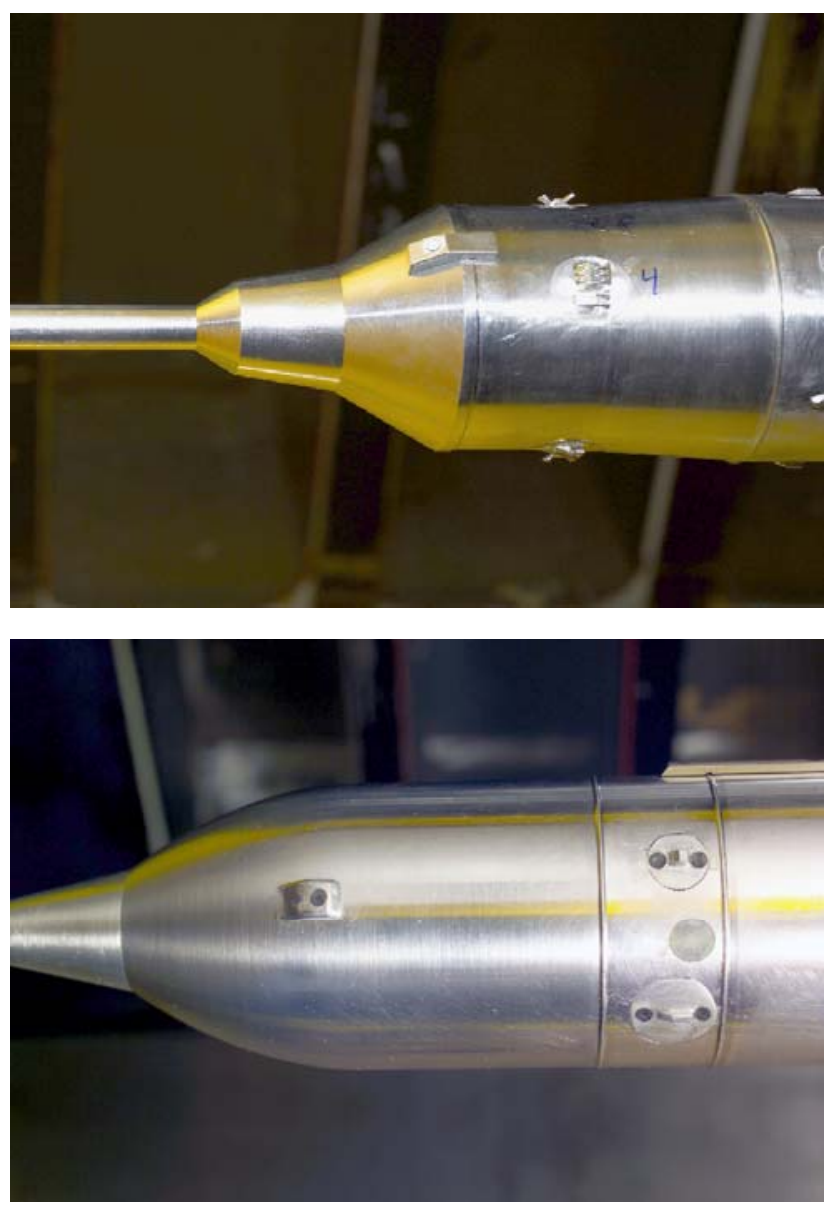

(b) Wind tunnel installation photographs of ADAC-2A model (top) and ADAC-2B model (bottom).

Fig. 42. Comparison of Ares I ADAC-2A and ADAC-2B BPC OMLs. 


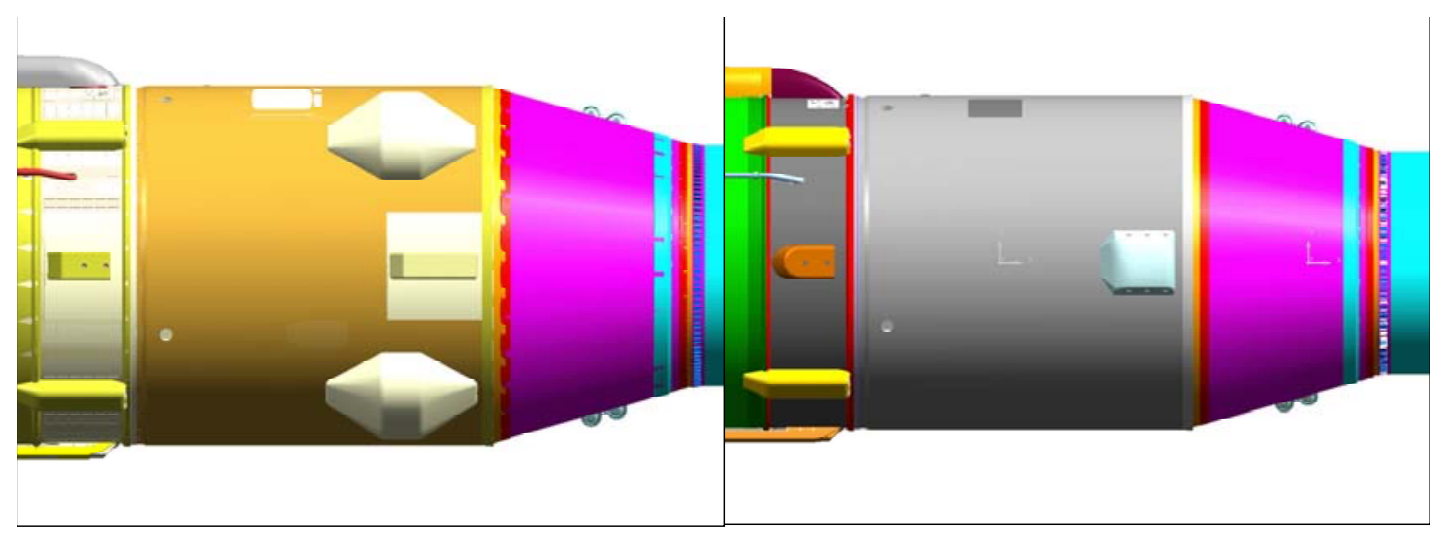

ADAC-2A

ADAC-2B

(a) CAD depiction of ADAC-2A with BDM covers (left) and ADAC-2B with BDM covers off (right).
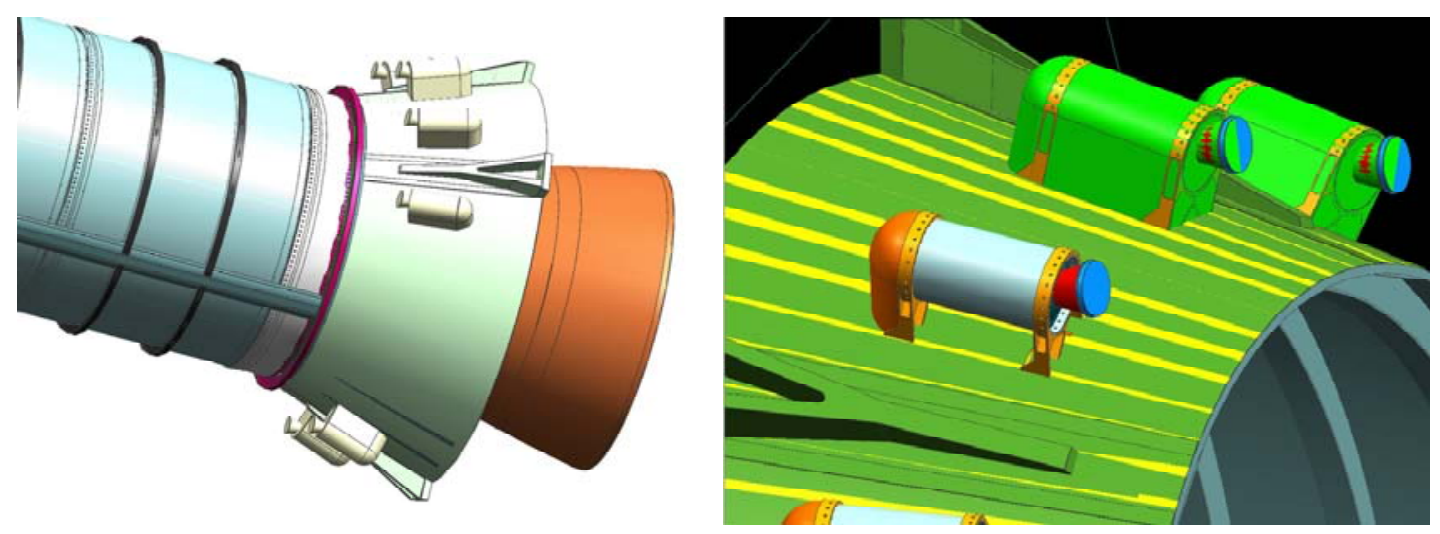

(b) CAD depiction of relocation of BDM covers to first stage aft skirt on ADAC-2B.

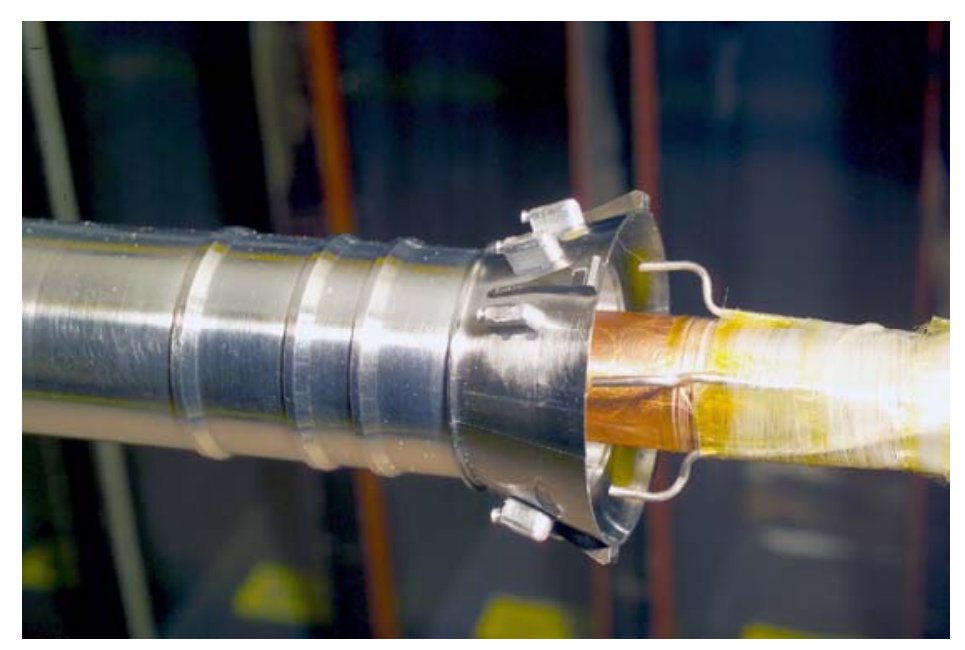

(c) Wind tunnel model installation photograph of BDM covers on first stage aft skirt of ADAC-2B.

Fig. 43. Details of BDM cover installation on the Ares I ADAC-2B. 


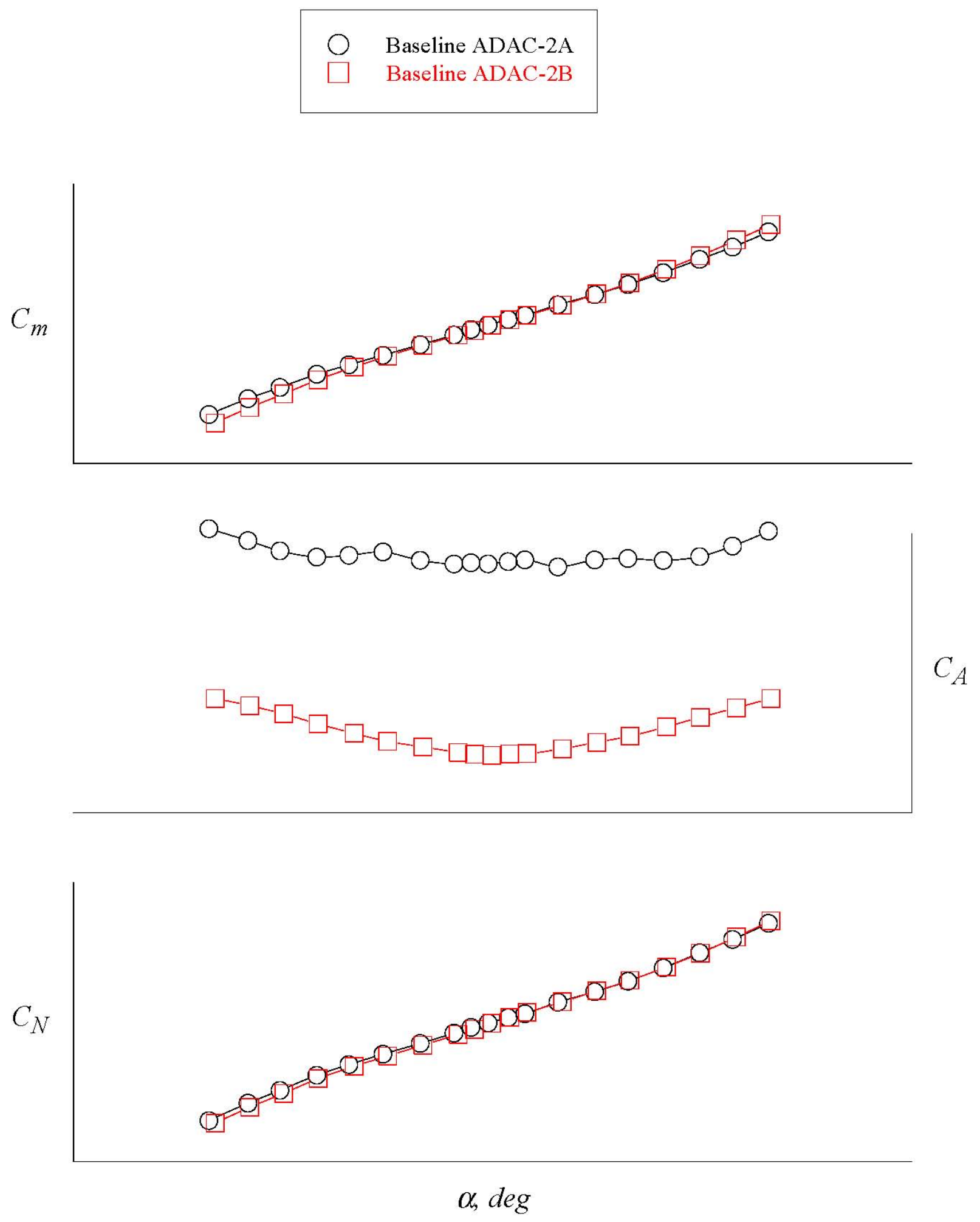

Fig. 44. Comparison of the longitudinal aerodynamic characteristics of the 0.01-scale Ares I ADAC-2A and Ares I ADAC-2B models at a selected supersonic Mach number in UPWT T. S. 1. 


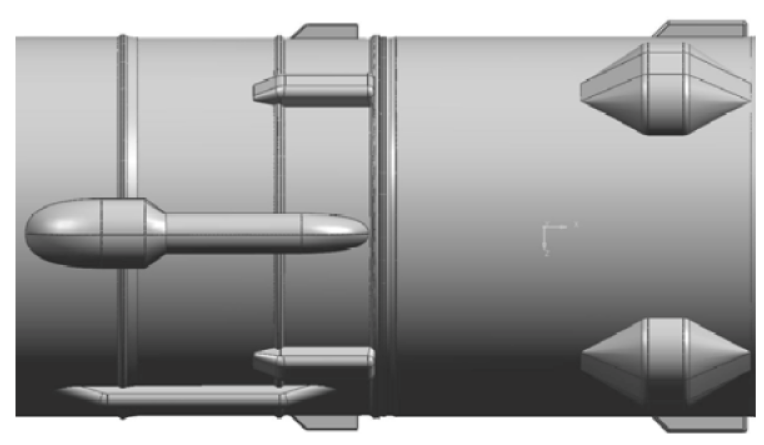

ADAC-2A

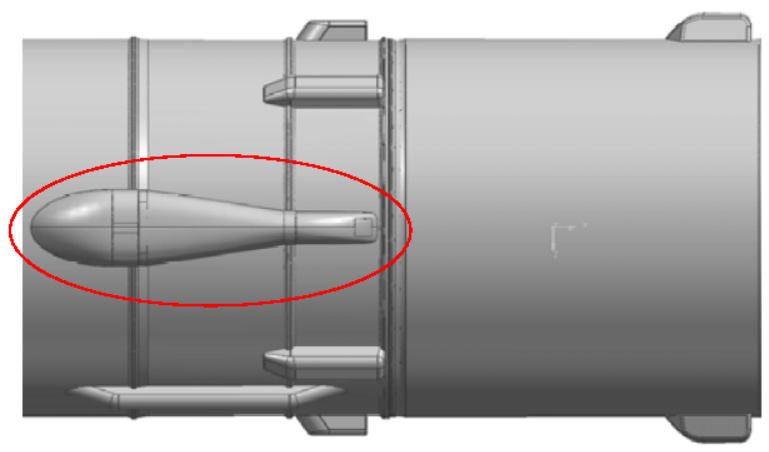

ADAC-2B

Fig. 45. CAD depiction of the LH2 feedline fairings on the Ares I ADAC-2A and ADAC-2B.

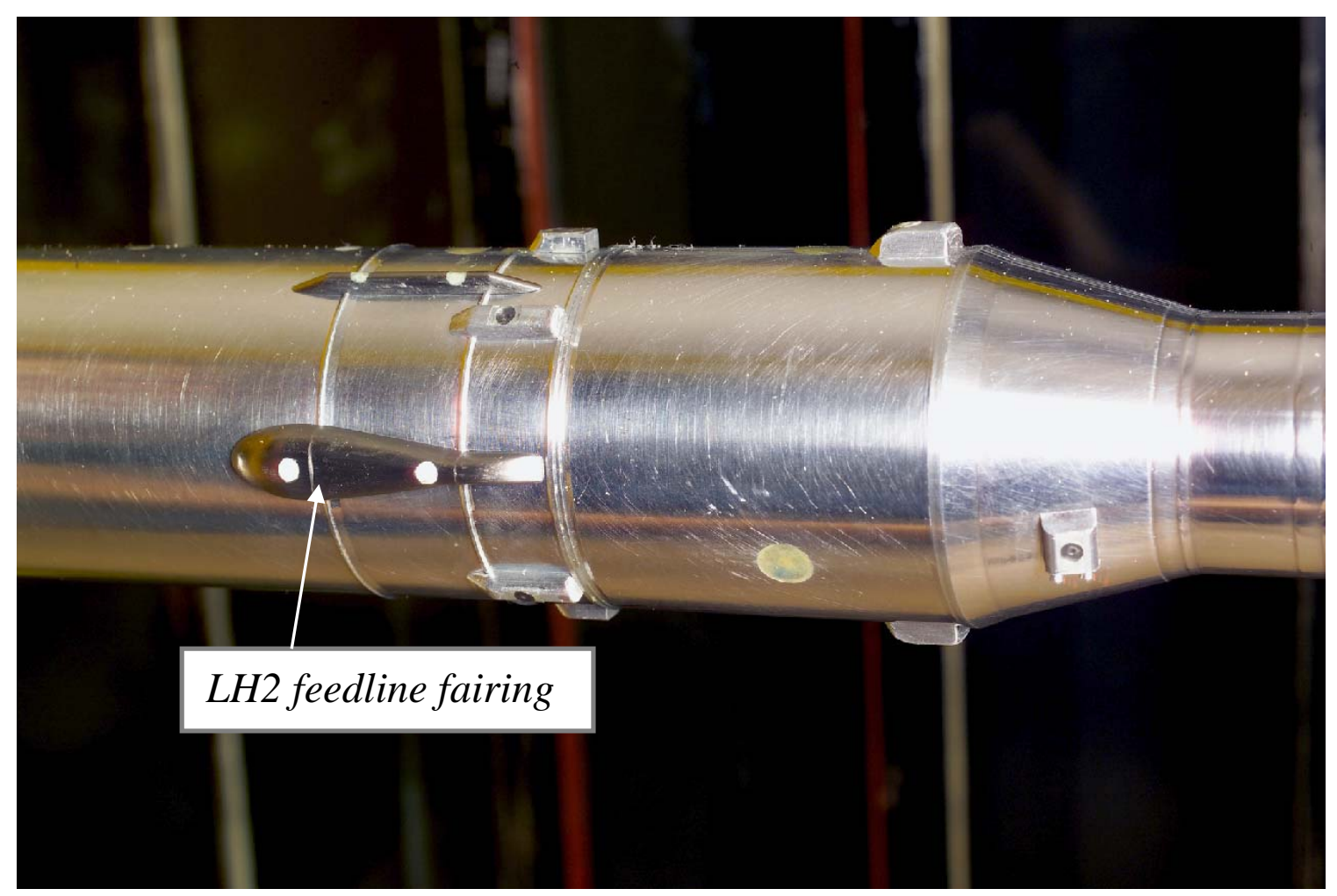

Fig. 46. Wind tunnel installation photograph of the LH2 feedline fairing on the 0.01-scale Ares I ADAC-2B model installed in UPWT T. S. 1. 

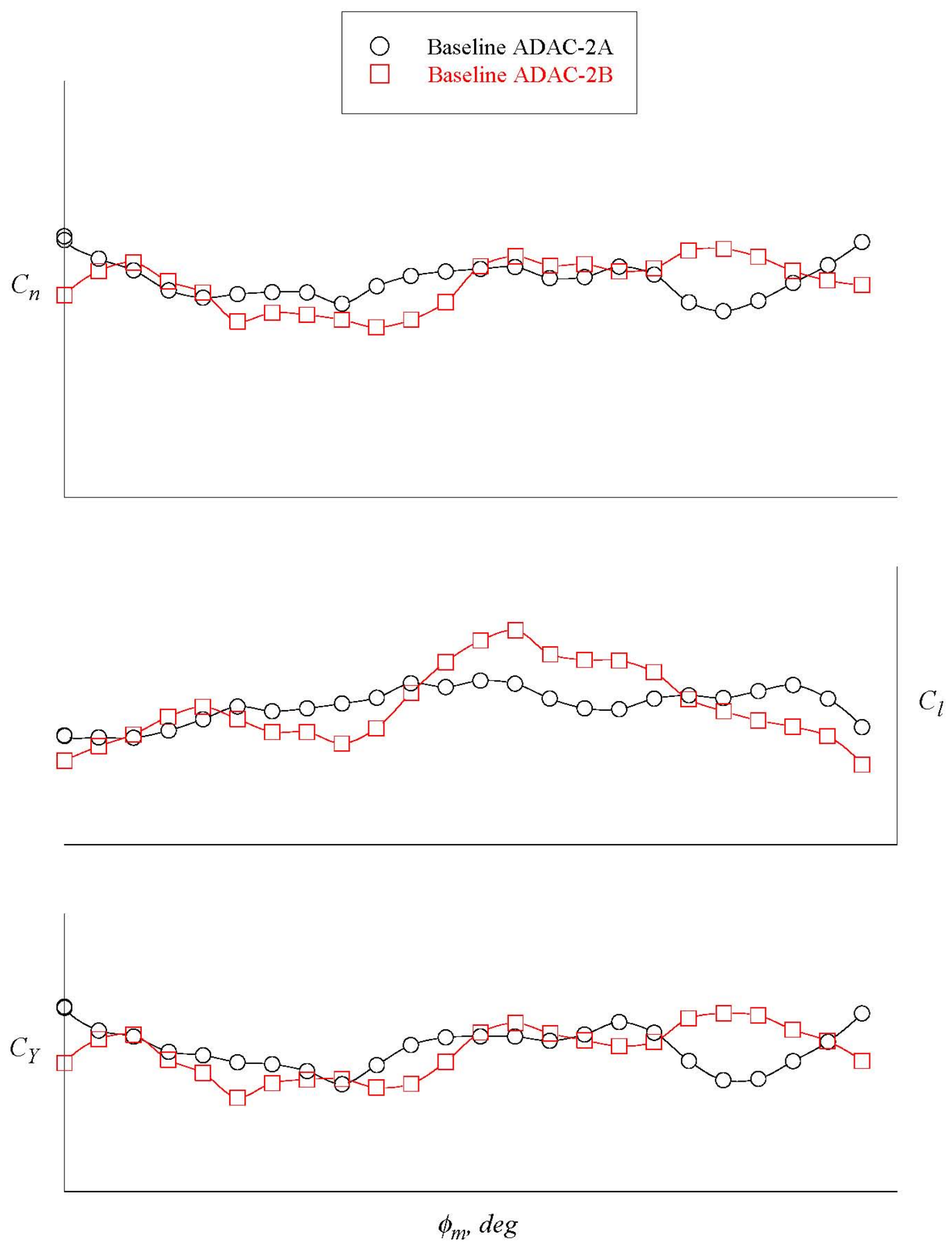

Fig. 47. Comparison of the lateral-directional aerodynamic characteristics of the 0.01-scale Ares I ADAC-2A and ADAC-2B models at a selected supersonic Mach number in UPWT T. S. 1. 


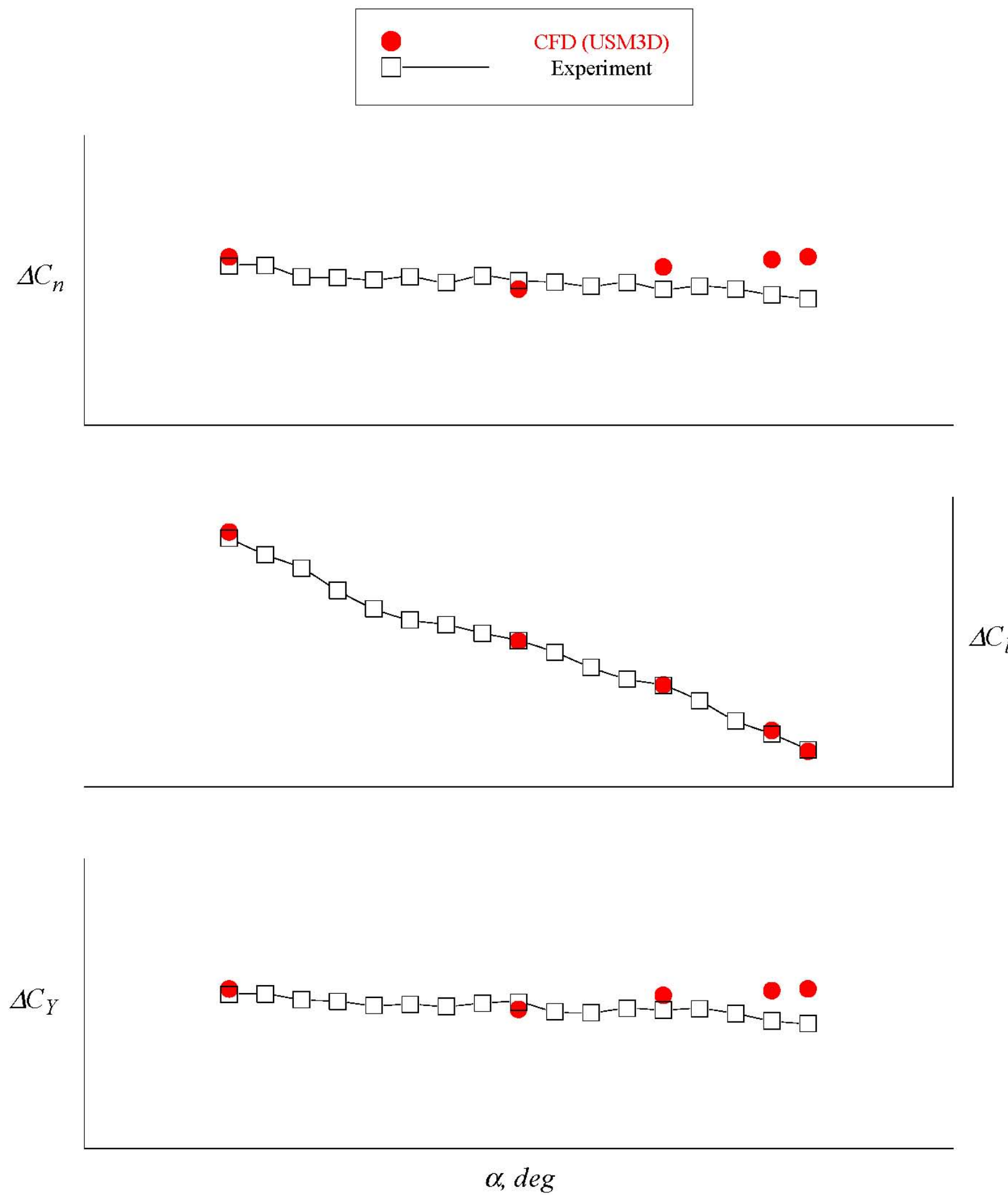

Fig. 48. Comparison of the experimental and CFD LH2 feedine fairing lateral-direction aerodynamic coefficient increments on the 0.01-scale Ares I ADAC-2B model in an angle of attack sweep at a selected supersonic Mach number in UPWT T. S. 1. 

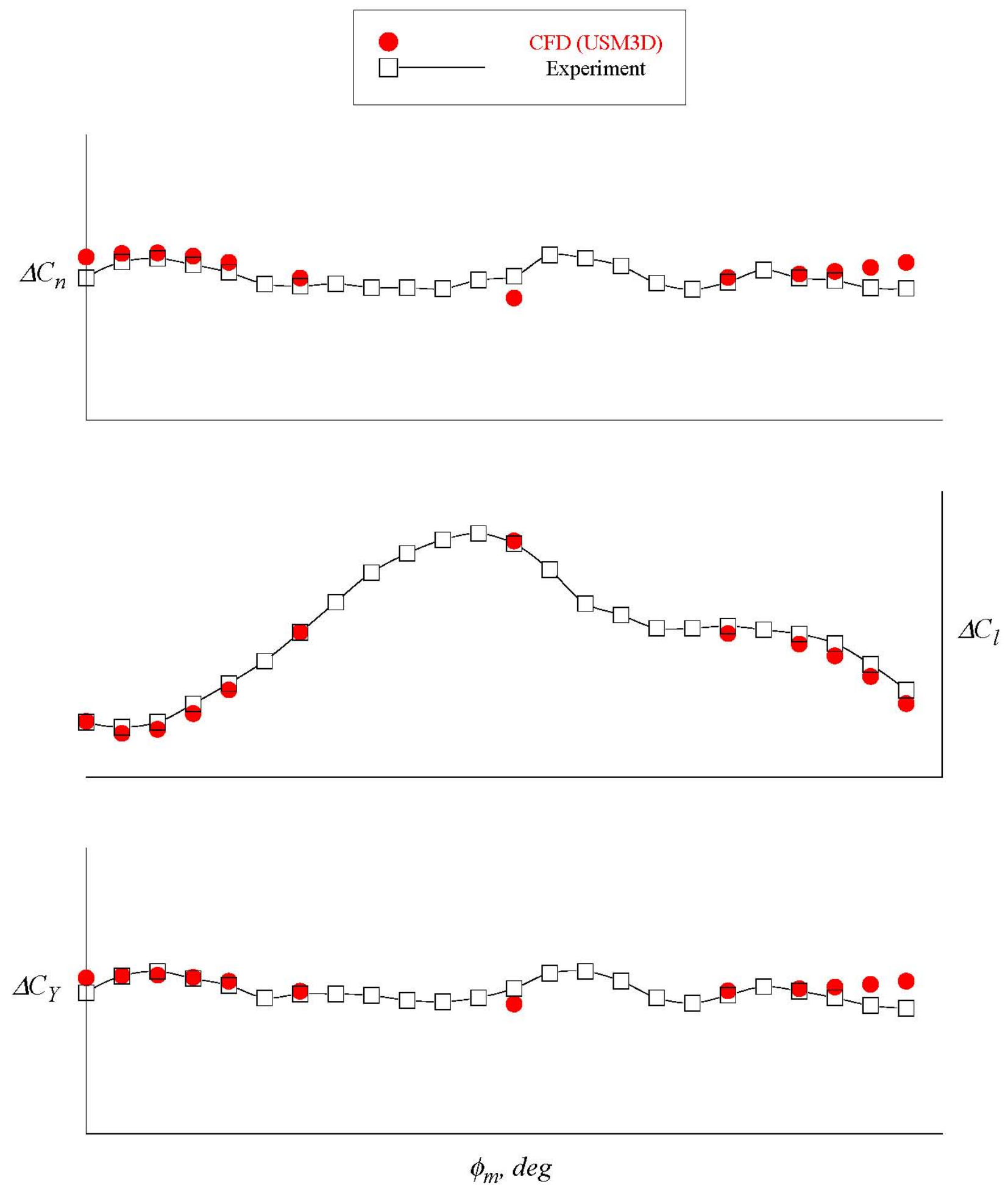

Fig. 49. Comparison of the experimental and CFD LH2 feedline fairing lateral-directional aerodynamic coefficient increments on the 0.01-scale Ares I ADAC-2B model in a roll angle sweep at a selected supersonic Mach number in UPWT T. S. 1. 


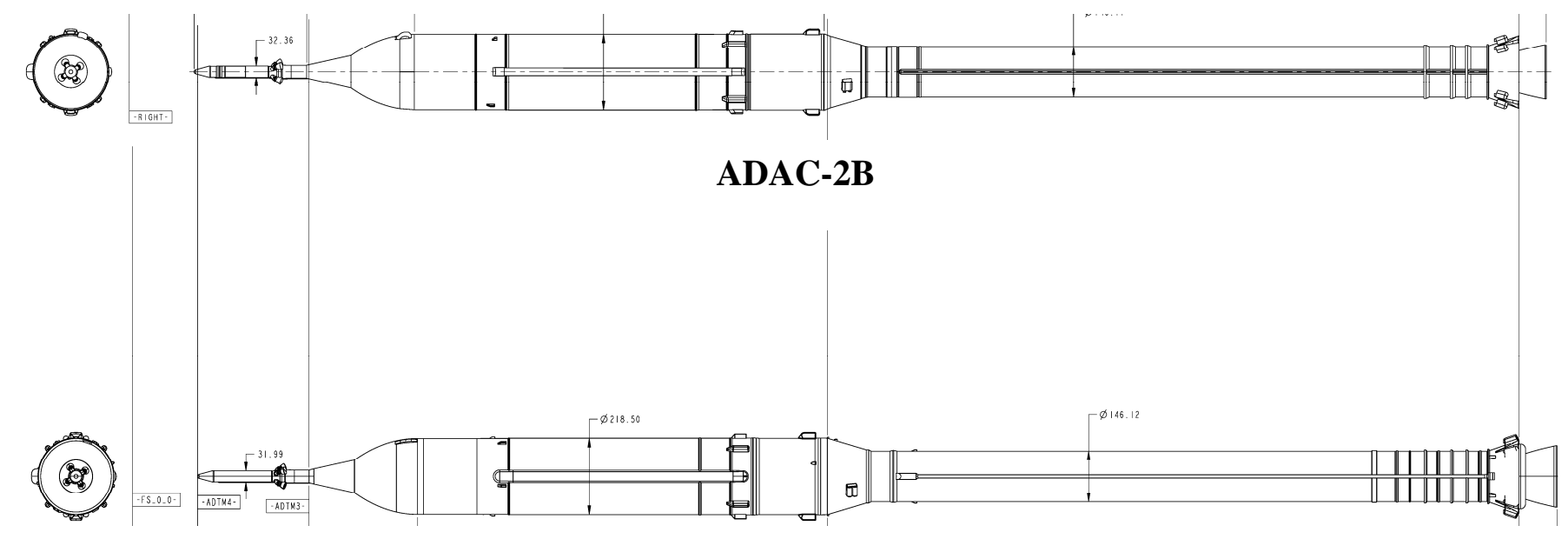

ADAC-3

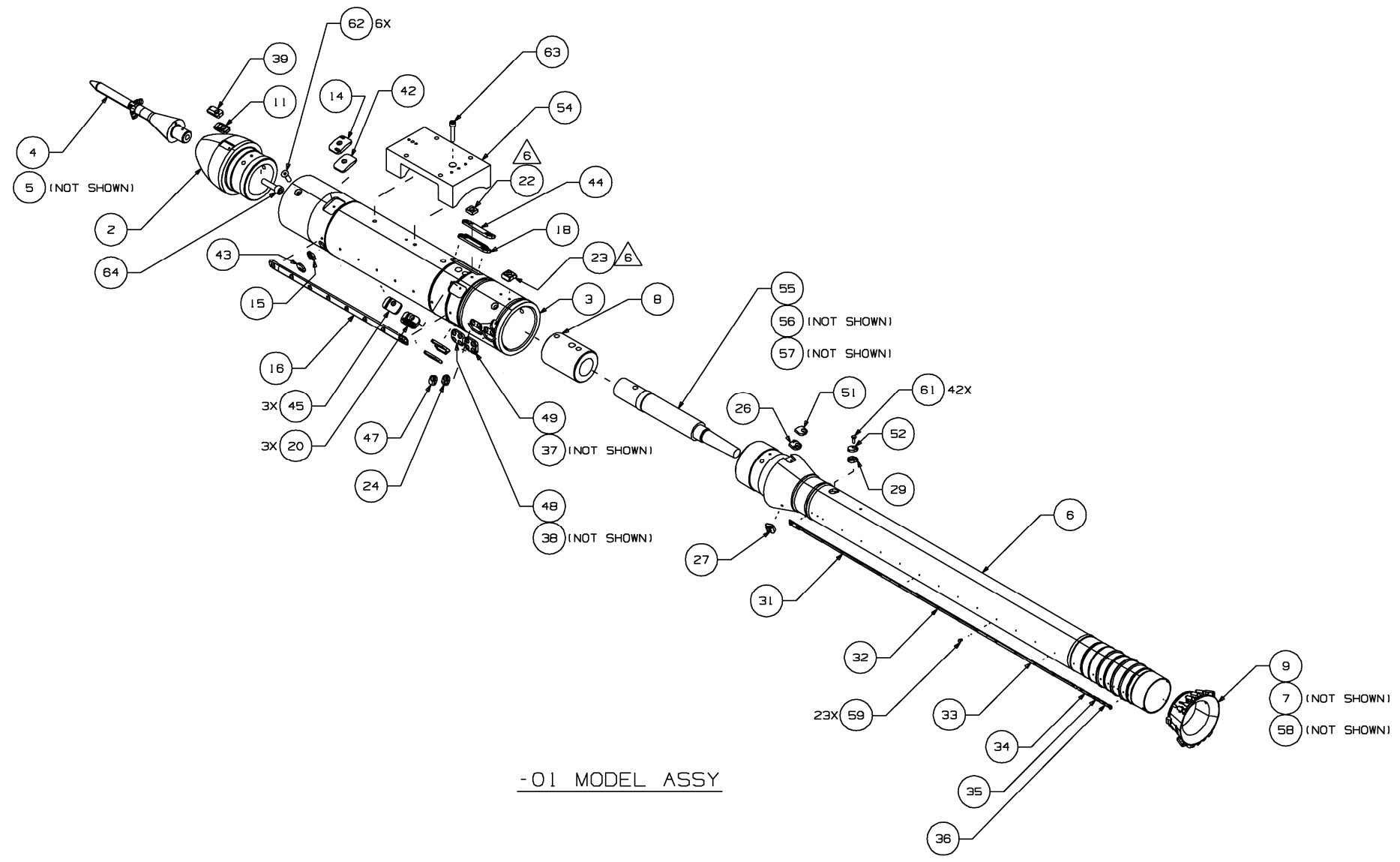

Fig. 50. Comparison of the Ares I ADAC-2B and Ares I ADAC-3 and assembly drawing of the 0.01-scale ADAC-3 force and moment model. 


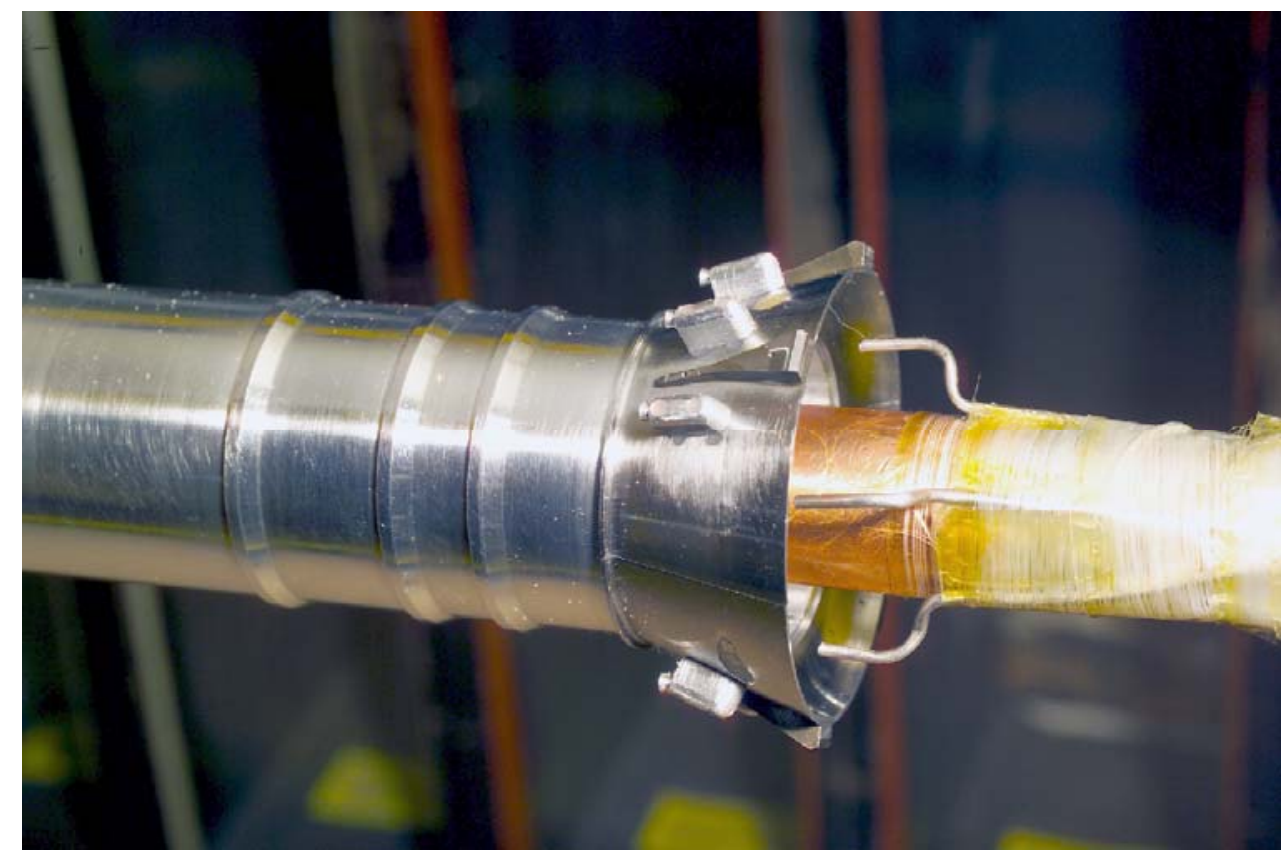

(a) Baseline ADAC-2B aft skirt.

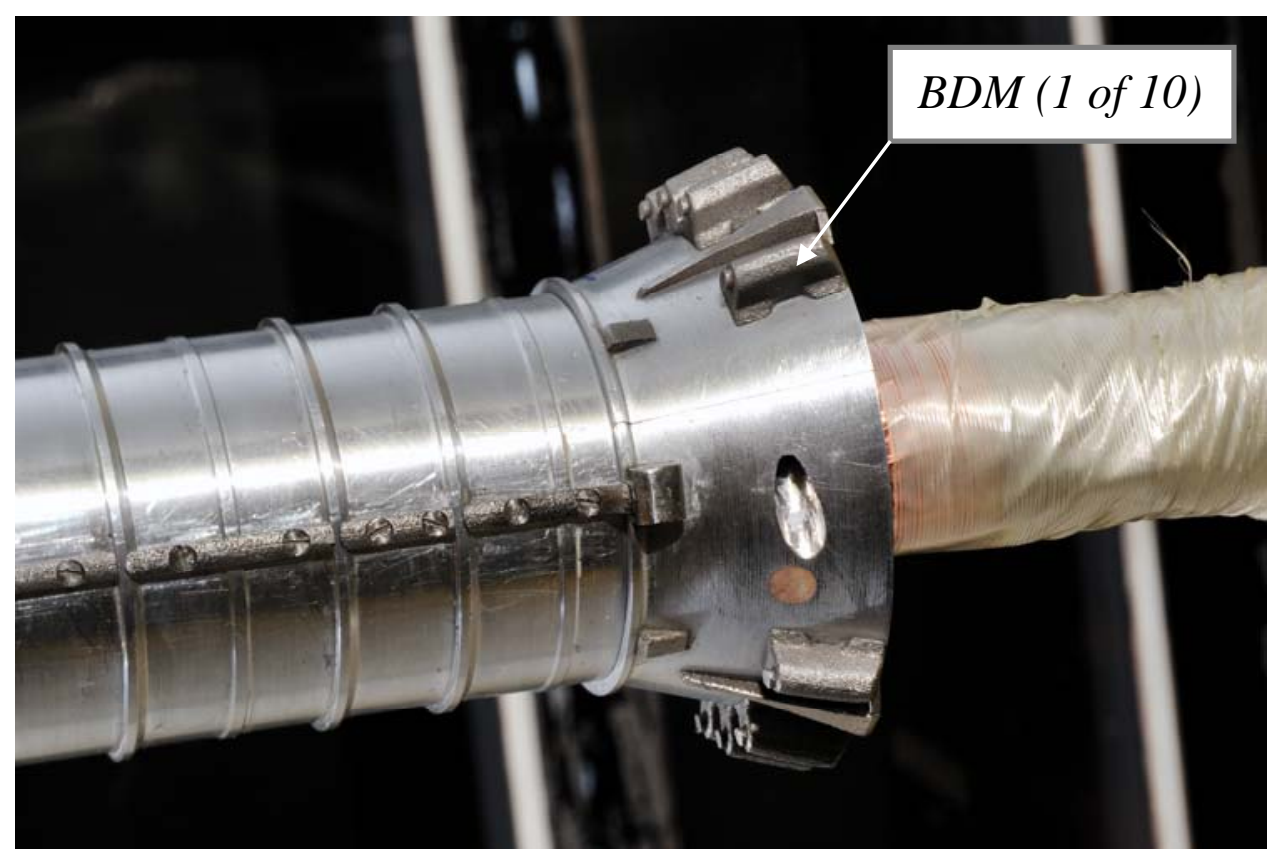

(b) Baseline ADAC-3 aft skirt.

Fig. 51. Wind tunnel installation photographs of the baseline 0.01-scale Ares I ADAC-2B and ADAC-3 first stage aft skirts in UPWT T. S. 1. 

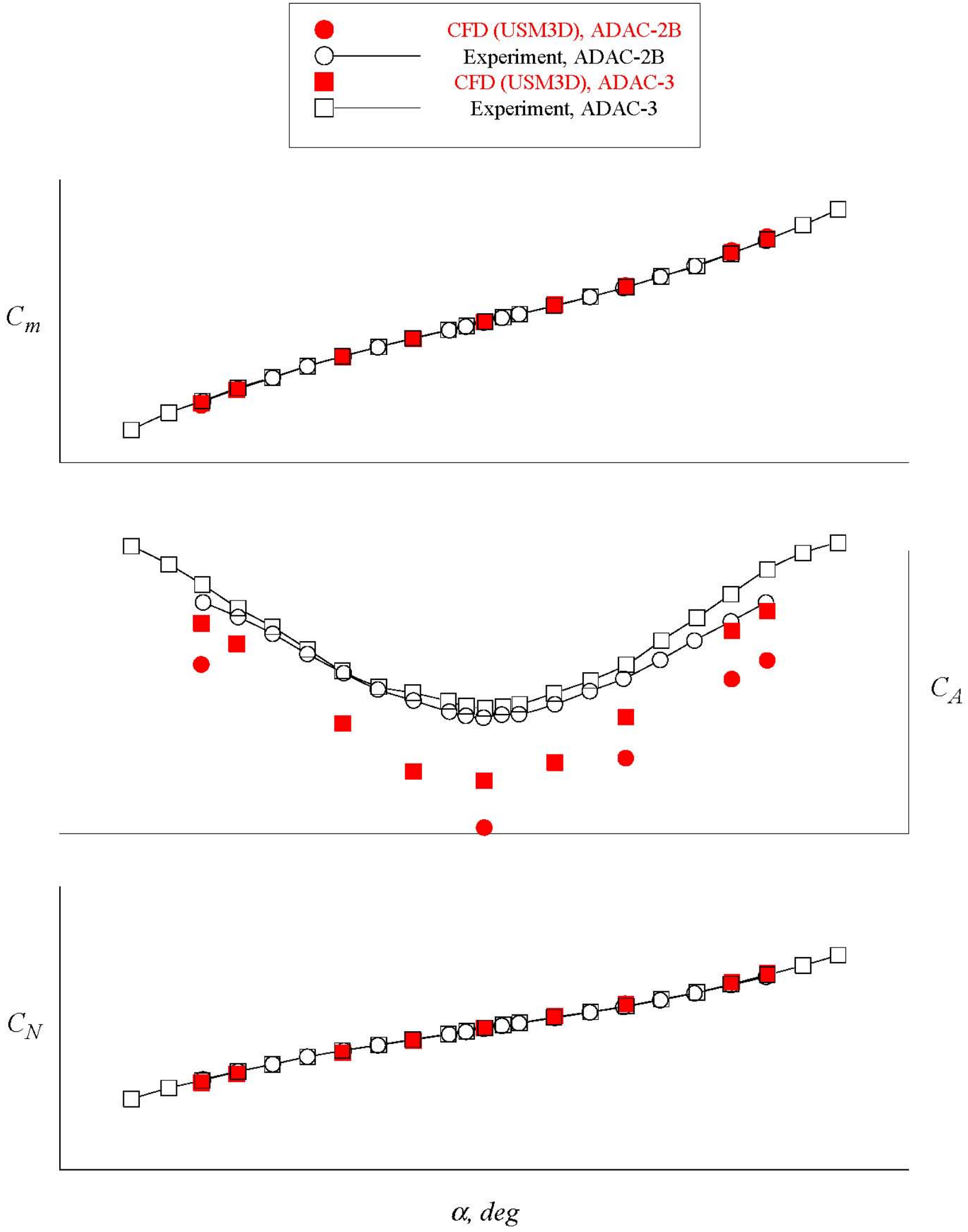

Fig. 52. Comparison of the longitudinal aerodynamic characteristics of the 0.01-scale Ares I ADAC-2B and ADAC-3B models in UPWT T. S. 1 and corresponding CFD predictions using USM3D. 


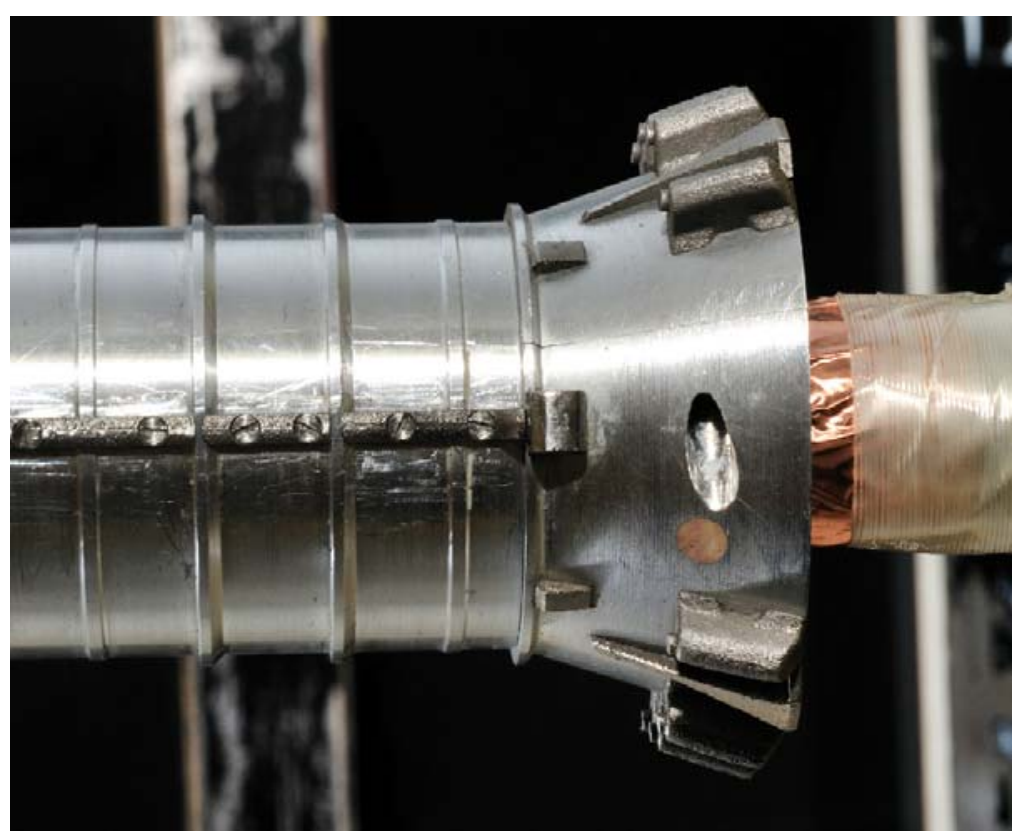

(a) Baseline aft skirt.

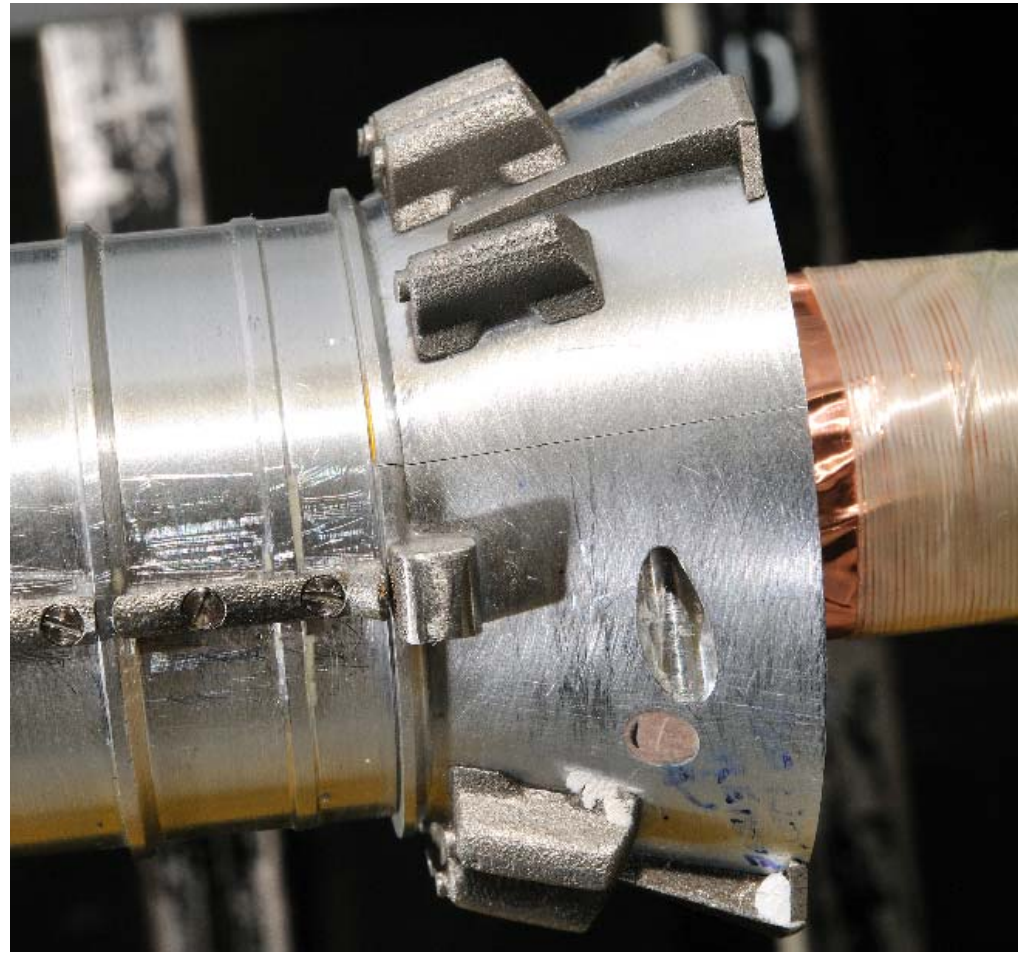

(b) Alternate aft skirt.

Fig. 53. Wind tunnel installation photographs of the baseline and alternate aft skirts on the 0.01-scale Ares I ADAC-3 wind tunnel model in UPWT T. S. 1. 

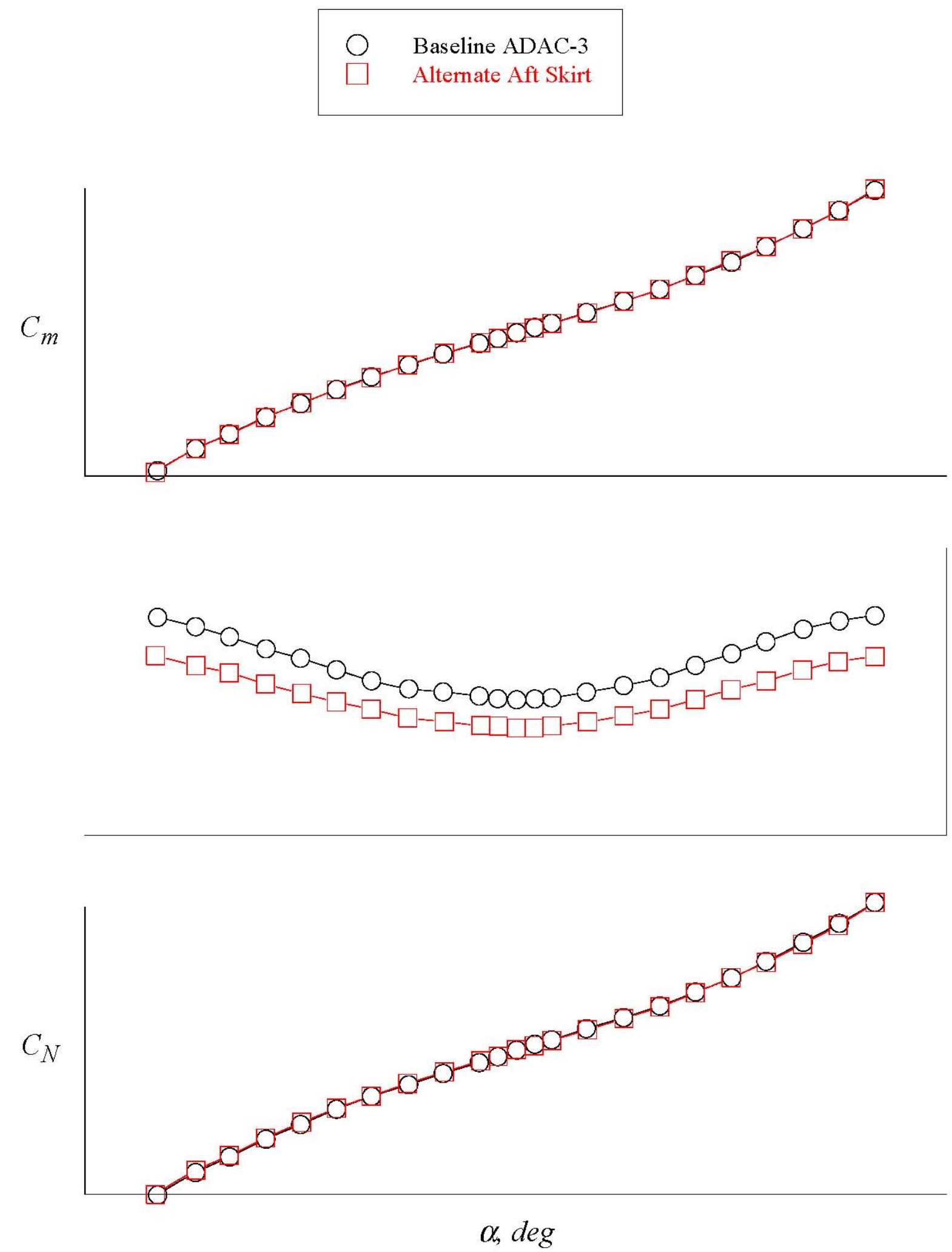

Fig. 54. Effect of the alternate aft skirt on the longitudinal aerodynamic characteristics of the 0.01 -scale Ares I ADAC-3 model at a selected supersonic Mach number in UPWT T. S. 1. 


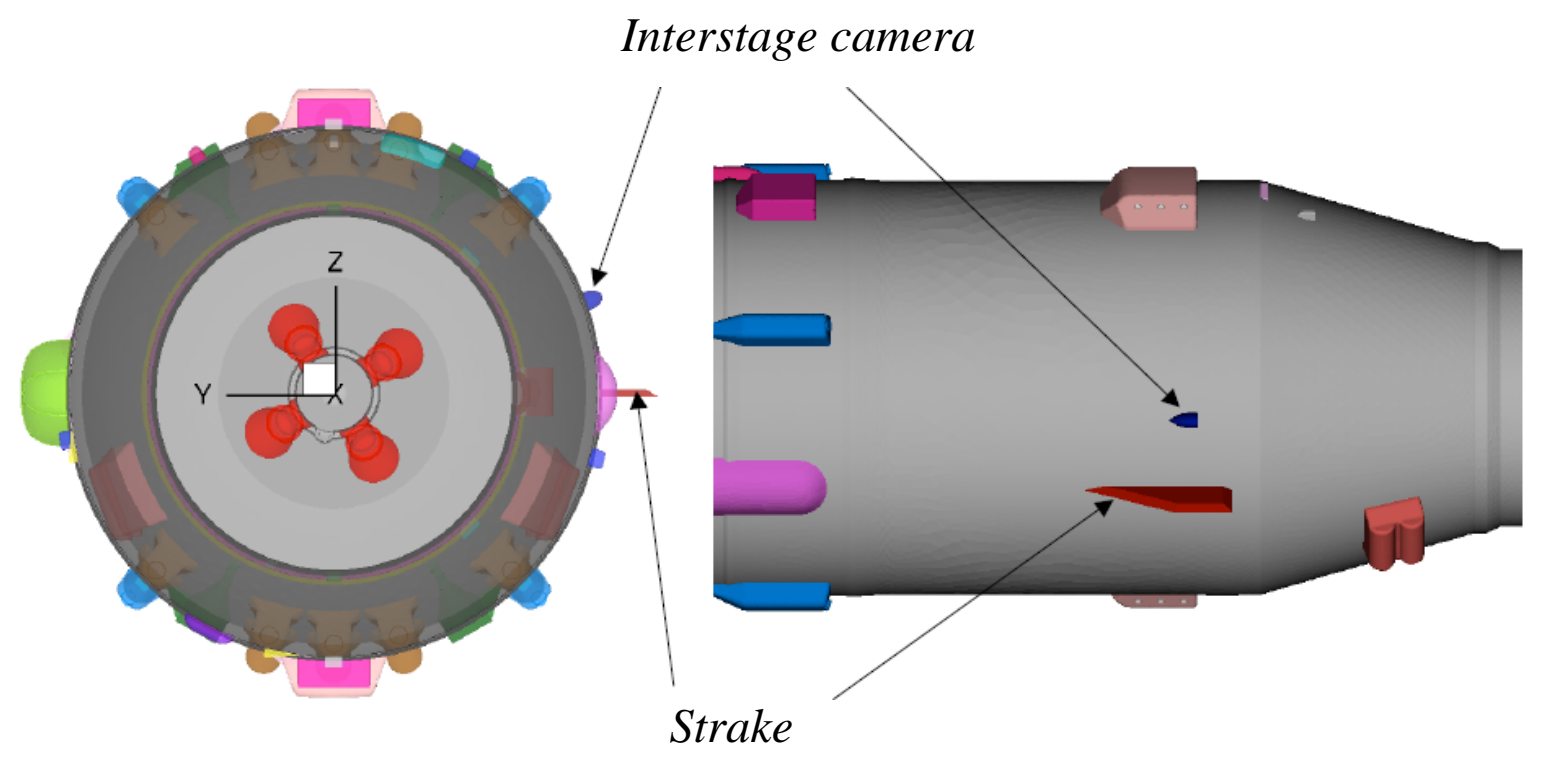

Fig. 55. CAD rendering of the roll control strake installed on the interstage of the Ares I ADAC-3.

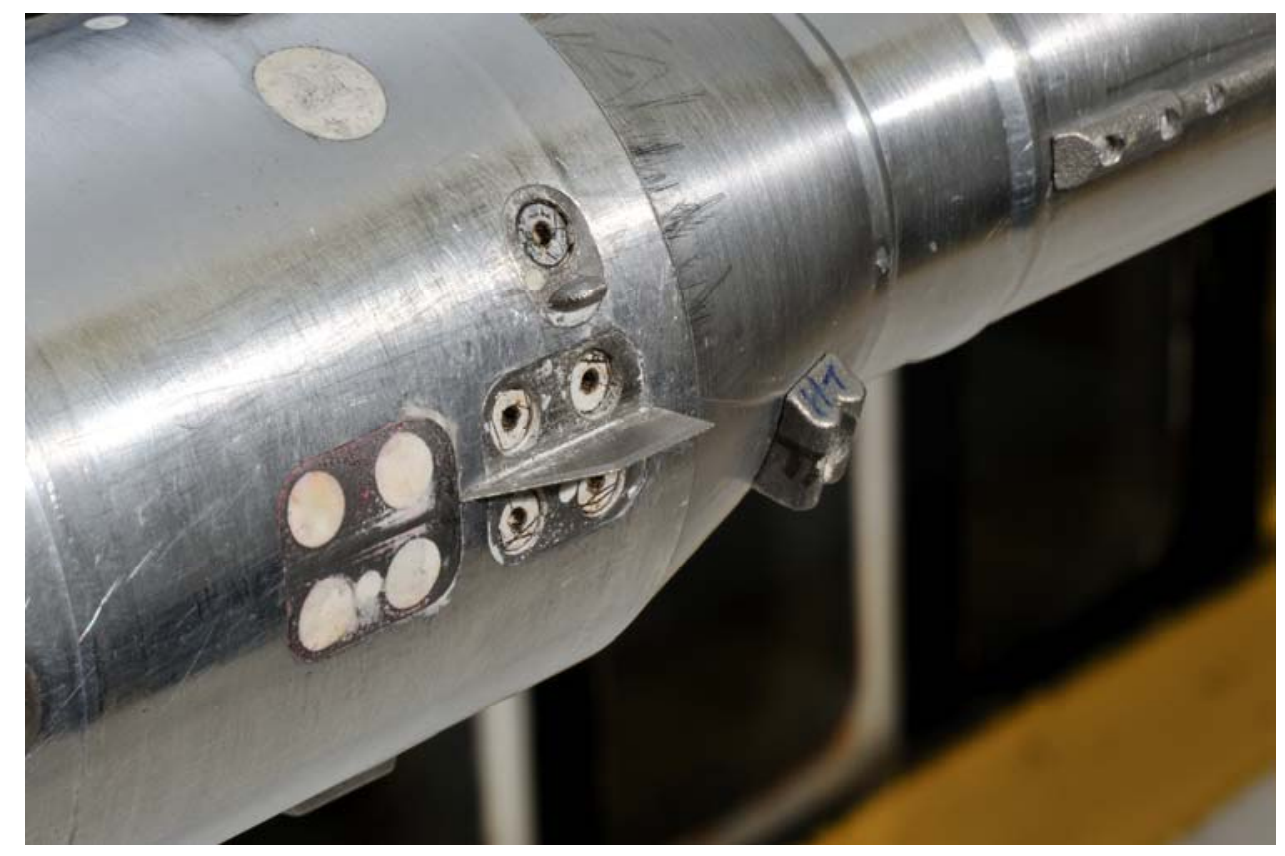

Fig. 56. Close-up photograph of the roll control strake on the 0.01-scale Ares I ADAC-3 model installed in UPWT T. S. 1. 

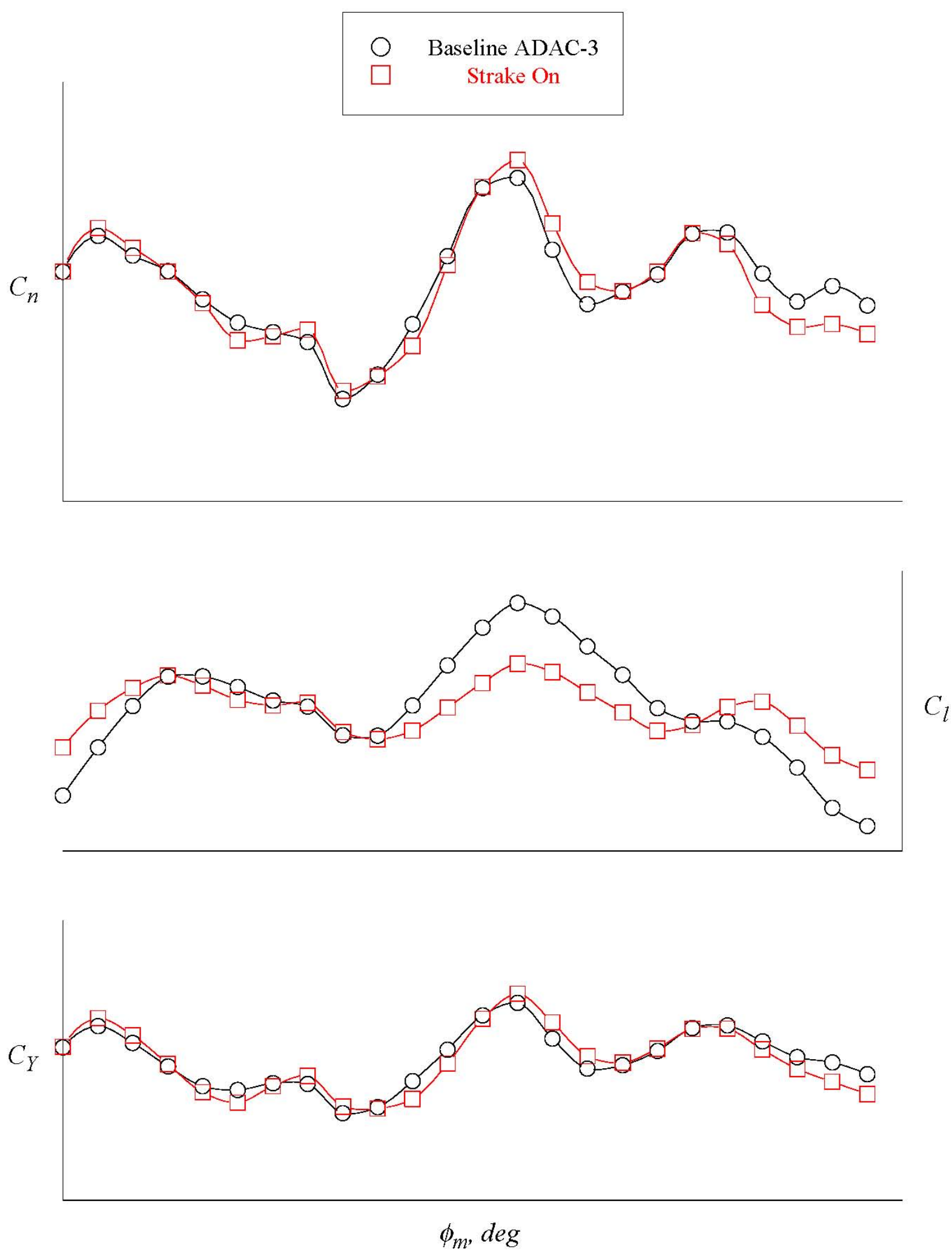

Fig. 57. Effect of the strake on the lateral-directional aerodynamic characteristics of the 0.01-scale Ares I ADAC-3 model in UPWT T. S. 1 in a roll sweep at fixed angle of attack. 


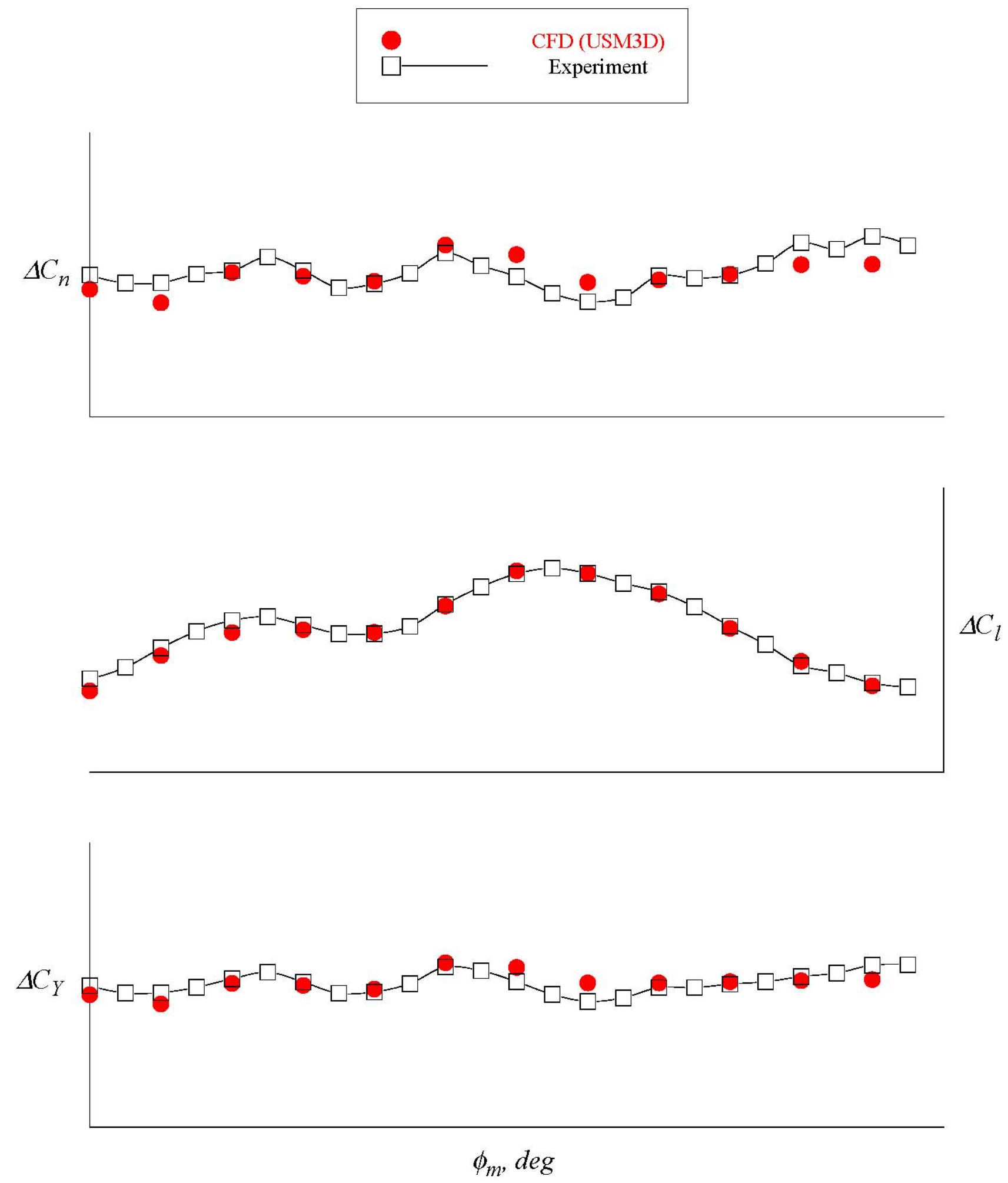

Fig. 58. Experimental and CFD lateral-directional aerodynamic coefficient increments due to the strake on the 0.01-scale Ares I ADAC-3 model in UPWT T. S. 1 in a roll sweep at fixed angle of attack. 


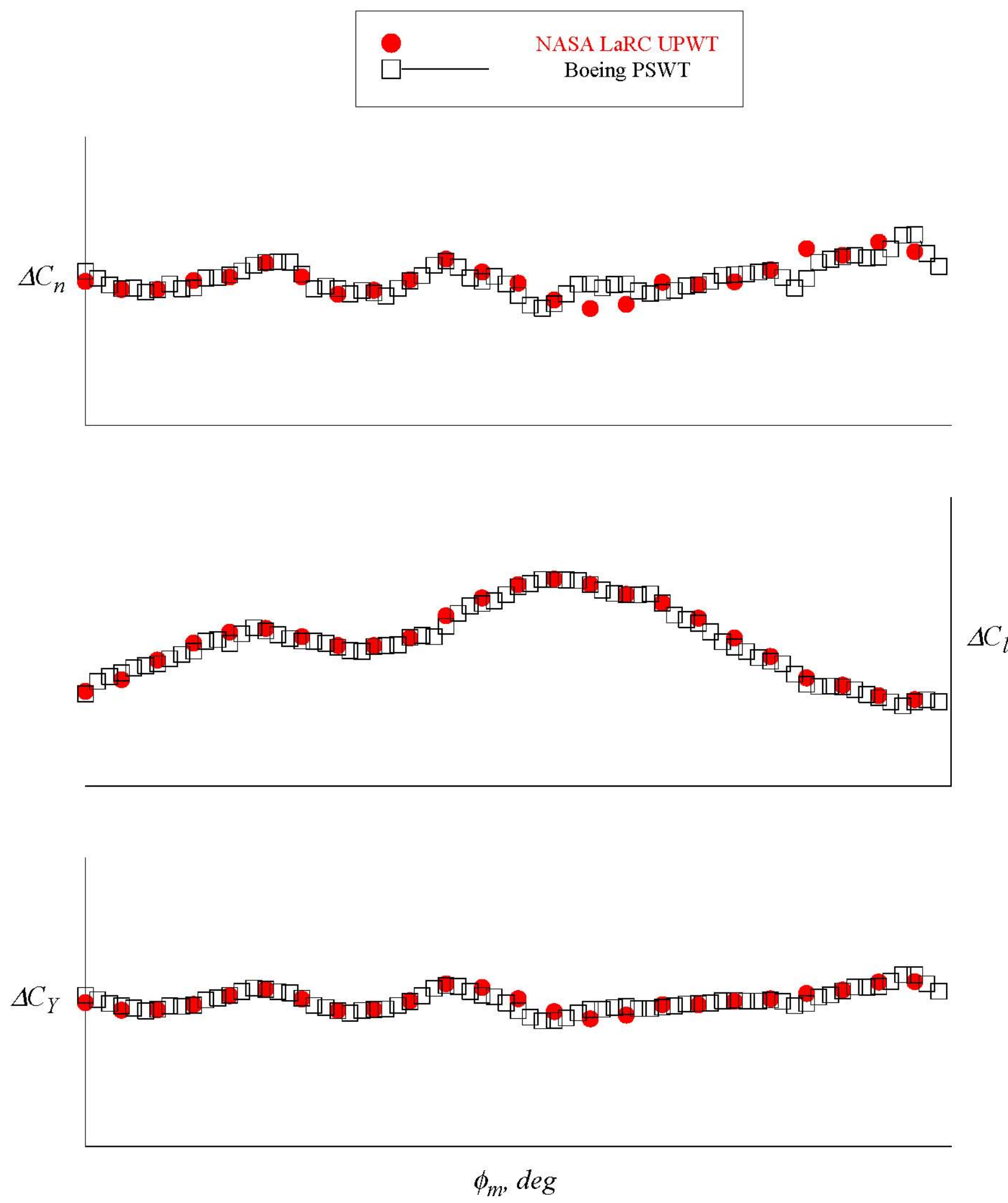

Fig. 59. Tunnel-to-tunnel comparison of the lateral-directional aerodynamic coefficient increments due to the strake on the 0.01-scale Ares I ADAC-3 model in a roll sweep at fixed angle of attack. 


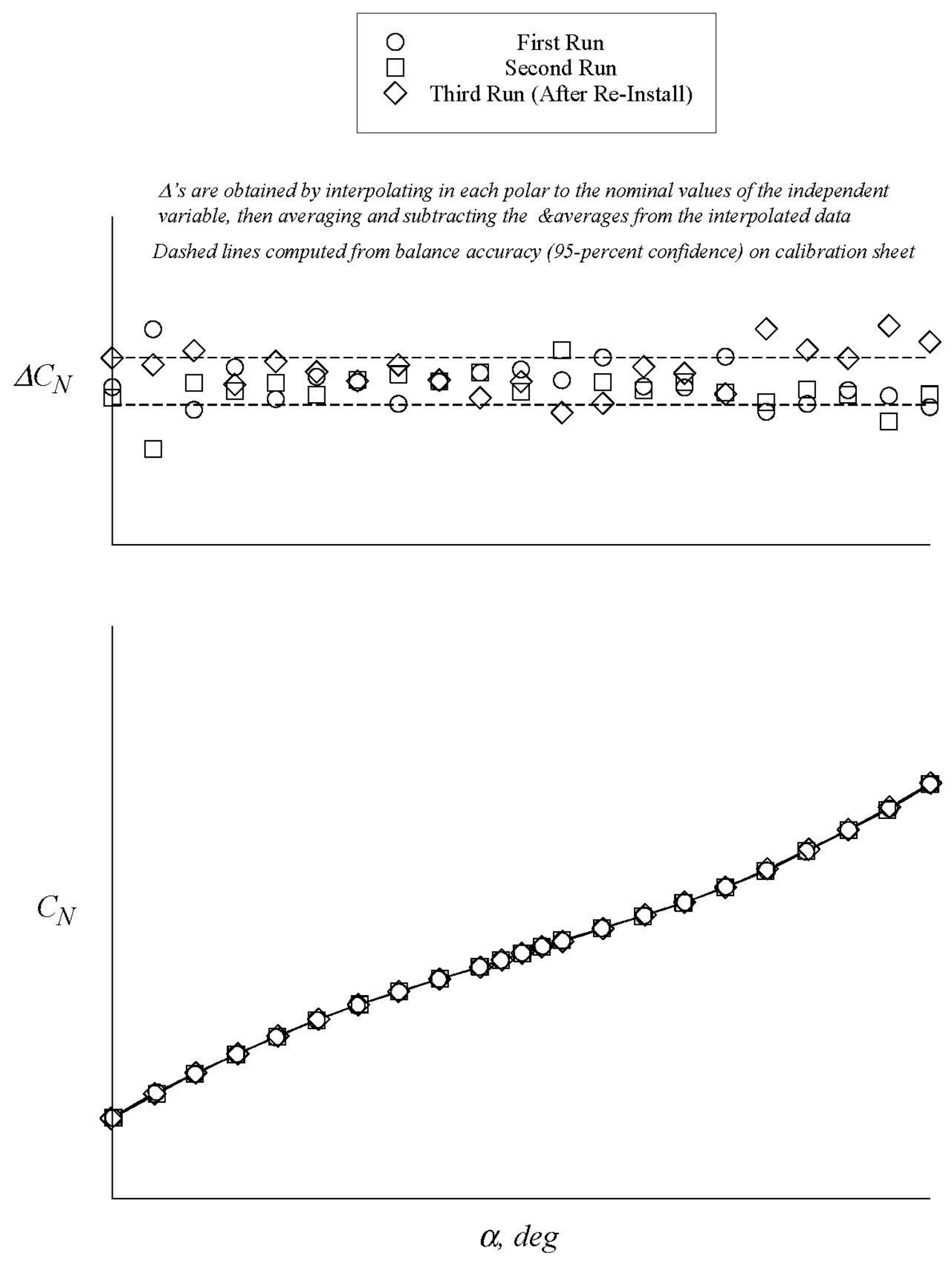

(a) Normal force coefficient

Fig. 60. Longer-term data repeatability assessment of the baseline 0.01-scale Ares I ADAC-3 model in UPWT T. S. 1 at a selected supersonic Mach number. 


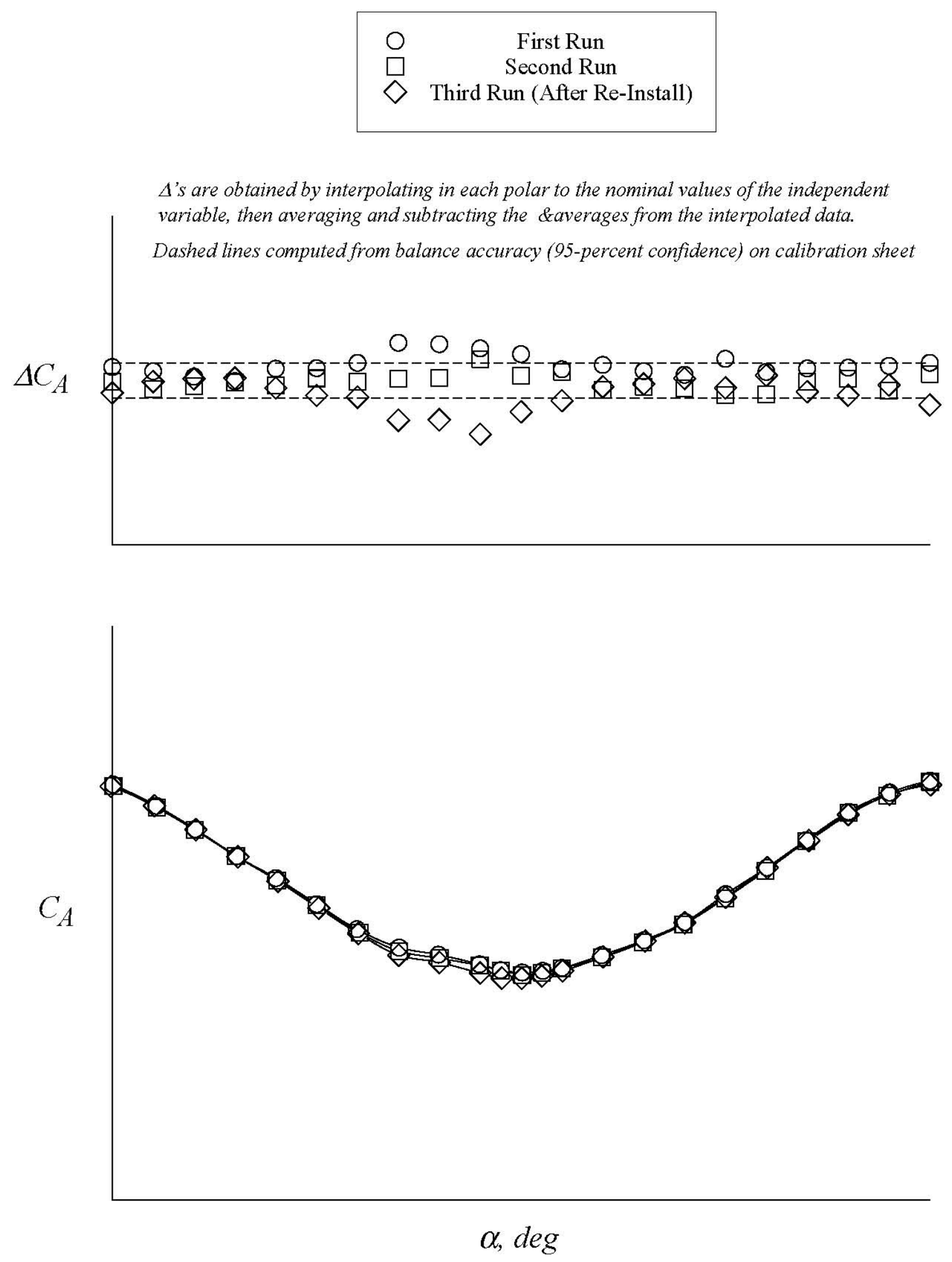

(b) Axial force coefficient

Fig. 60. Longer-term data repeatability assessment of the baseline 0.01-scale Ares I ADAC-3 model in UPWT T. S. 1 at a selected supersonic Mach number. 


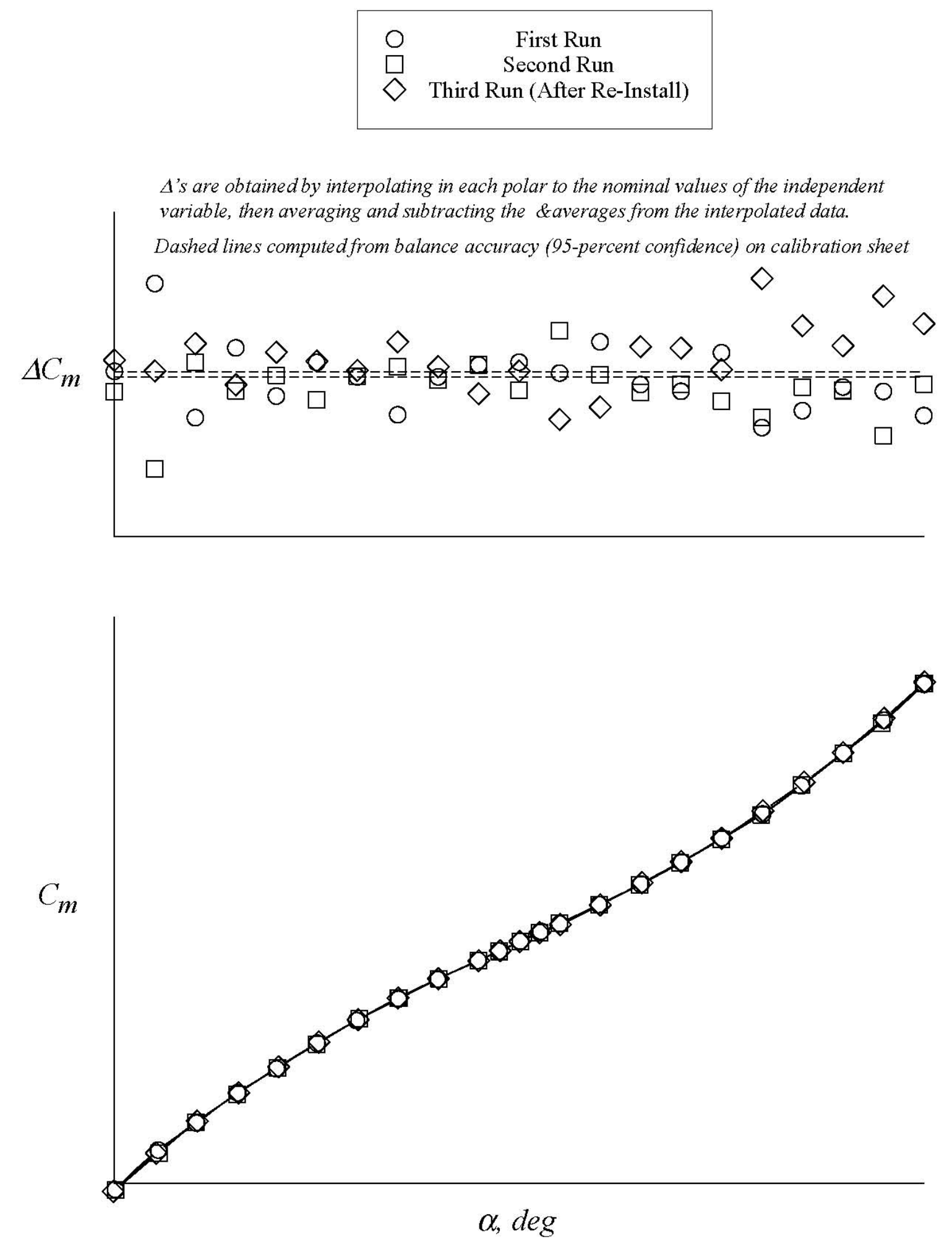

(c) Pitching moment coefficient.

Fig. 60. Longer-term data repeatability assessment of the baseline 0.01-scale Ares I ADAC-3 model in UPWT T. S. 1 at a selected supersonic Mach number. 


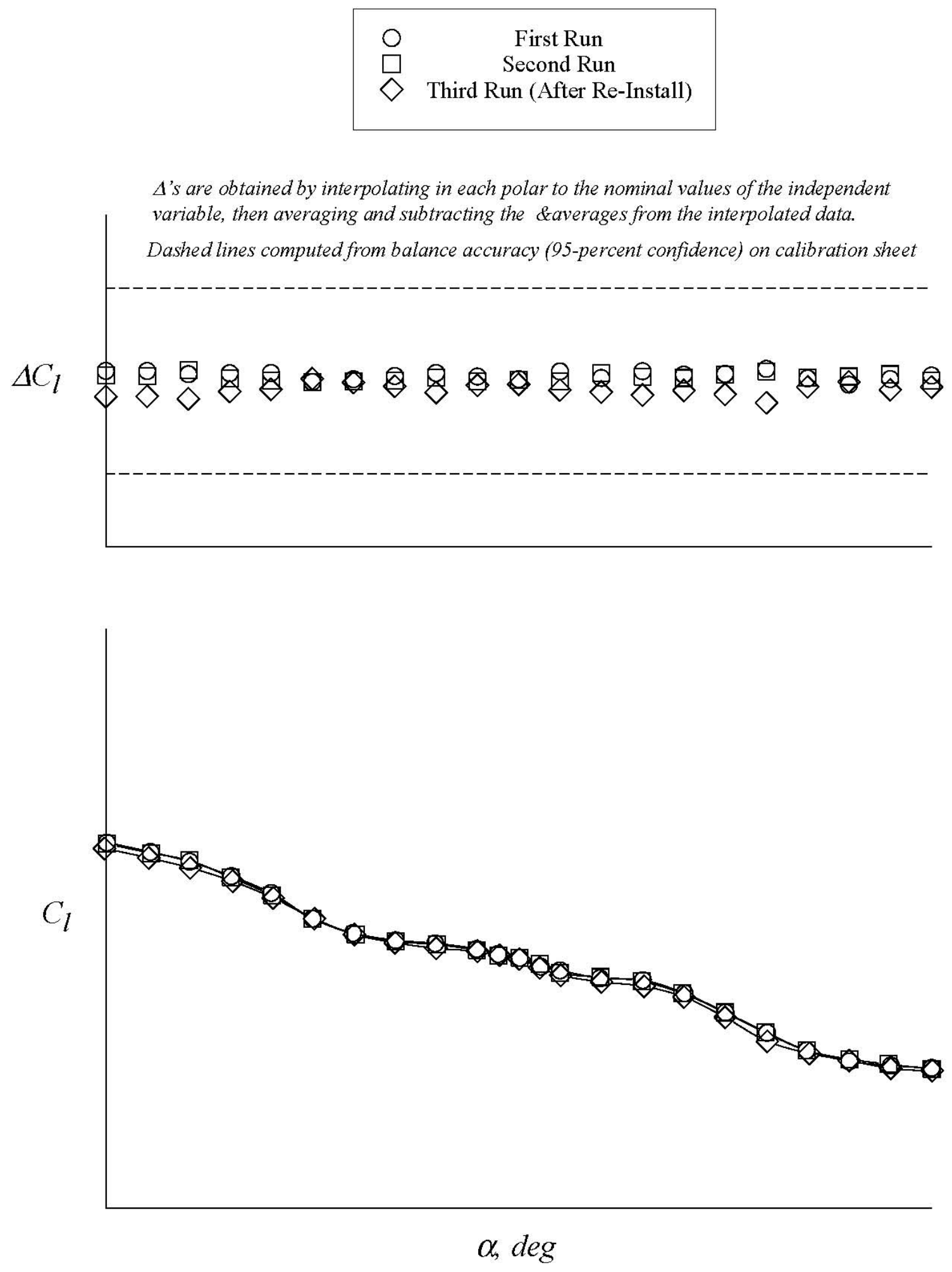

(d) Rolling moment coefficient.

Fig. 60. Longer-term data repeatability assessment of the baseline 0.01-scale Ares I ADAC-3 model in UPWT T. S. 1 at a selected supersonic Mach number.

American Institute of Aeronautics and Astronautics 

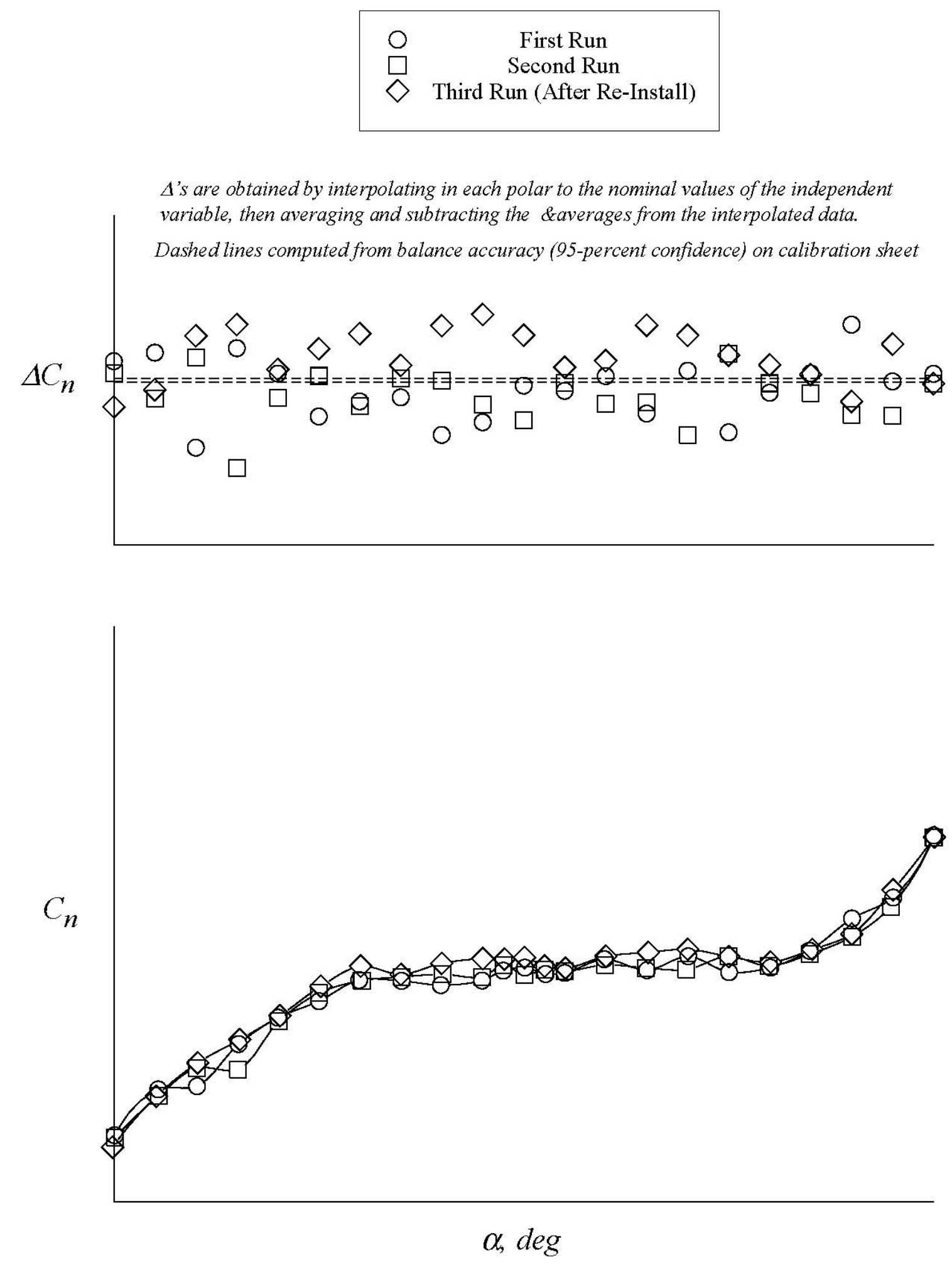

(e) Yawing moment coefficient.

Fig. 60. Longer-term data repeatability assessment of the baseline 0.01-scale Ares I ADAC-3 model in UPWT T. S. 1 at a selected supersonic Mach number. 


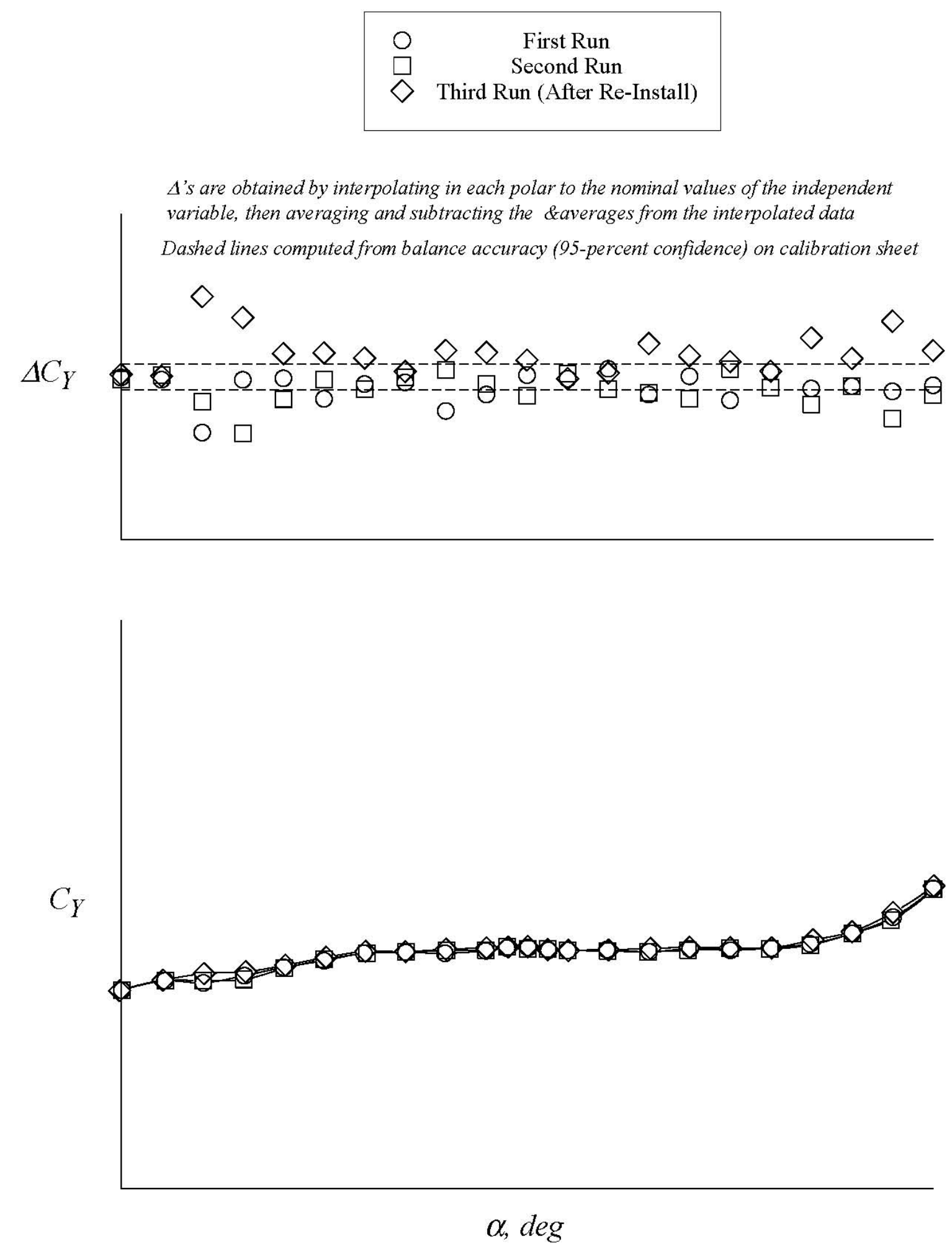

(f) Side force coefficient.

Fig. 60. Longer-term data repeatability assessment of the baseline 0.01-scale Ares I ADAC-3 model in UPWT T. S. 1 at a selected supersonic Mach number. 